Aus dem Institut für Allgemeinmedizin

(Prof. Dr. E. Hummers-Pradier)

der Medizinischen Fakultät der Universität Göttingen

\title{
Vorbilder und prägende Personen im Medizinstudium
}

\author{
INAUGURAL-DISSERTATION
}

zur Erlangung des Doktorgrades

der Medizinischen Fakultät

der Georg-August-Universität zu Göttingen

\author{
vorgelegt von \\ Jenny Katharina Bücken \\ aus \\ Düsseldorf
}

Göttingen 2016 
Dekan:

1. Berichterstatter:

2. Berichterstatter/in:
Prof. Dr. rer. nat. H. K. Kroemer

Prof. Dr. disc. pol. W. Himmel

Prof. Dr. med. T. Raupach

Tag der mündlichen Prüfung: 11.10.2017 


\section{Inhaltsverzeichnis}

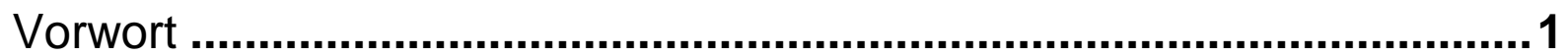

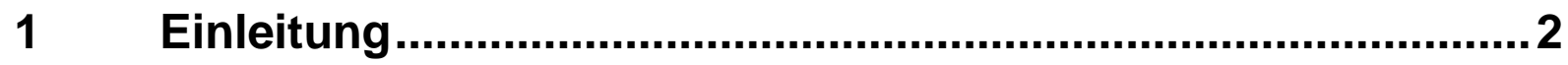

2 Stand der Forschung ....................................................... 4

$3 \quad$ Fragestellung und Ziel ...................................................... 7

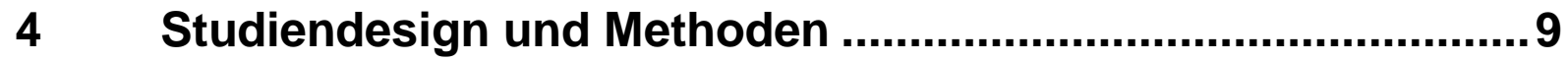

4.1 Erster Studienteil: Auswertung der ELSAS-Daten .................................. 9

4.1.1 Studienteilnehmer und Fragebogen der ELSAS-Studie.............................. 9

4.1.2 Wahl der Auswertungsmethode des ersten Teils ...................................... 9

4.1.3 Charakterisierung des Materials ............................................................ 10

4.1.4 Zusammenfassende Inhaltsanalyse nach Mayring …............................... 10

4.1.5 Reduktion des Materials ................................................................. 11

4.2 Zweiter Studienteil: Narrative Interviews ............................................. 14

4.2.1 Begründung für die Ausweitung der Studie........................................... 14

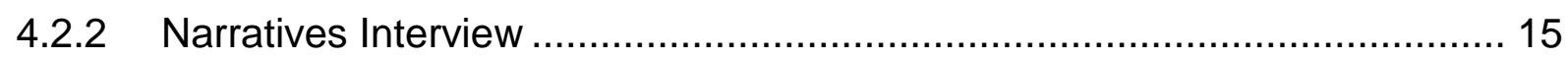

4.2.3 Rekrutierung und Vorbereitung der Studienteilnehmer/innen .................... 15

4.2.4 Durchführung der Interviews ........................................................... 16

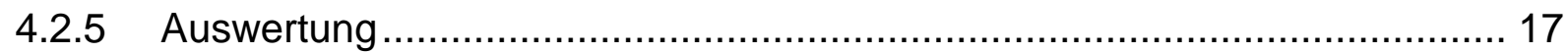

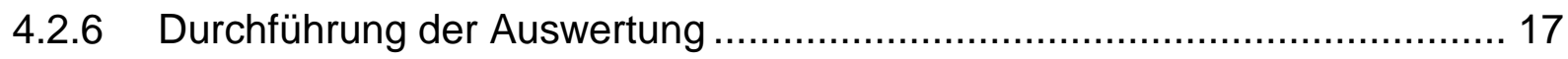

4.3 Datenschutz und Genehmigung durch die Ethikkommission ............... 23

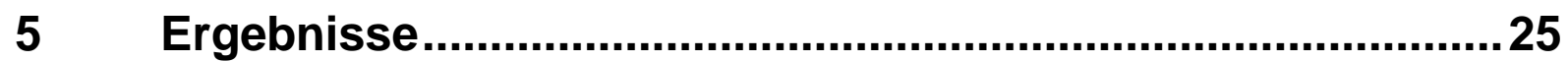

5.1 Hauptkategorien der ELSAS-Studie ...................................................... 25

5.2 Ergebnisse der Interviewauswertung …................................................ 29

5.2.1 Orientierungshilfen durch Vorbilder: Die Rolle der Kommilitonen ............... 29

5.2.2 Orientierungshilfen durch Vorbilder: Die Rolle von Lehrenden im Studium

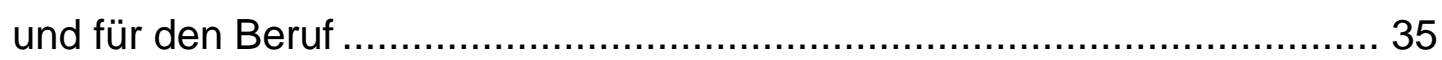

5.2.3 Identifikation mit Ärzten, Studium und Beruf ......................................... 42

5.2.4 Auseinandersetzung mit Idealen der ärztlichen Profession ....................... 48

5.2.5 Rolleneinübung: Auseinandersetzung mit der Rolle als Medizinstudent/in

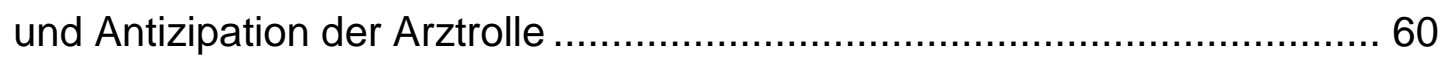

5.2.6 Entwicklung von Strategien zur Bewältigung des Studiums........................ 70 


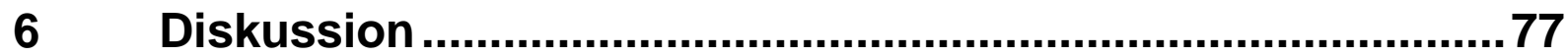

6.1 Zusammenfassung der Ergebnisse ……........................................... 77

6.2 Vergleich mit der Literatur .................................................................. 78

6.2.1 Schilderung negativer Erfahrungen................................................... 79

6.2.2 Medizinkultur und implizites Lernen ................................................... 79

6.2.3 Einfluss der aktuellen Situation der Studierenden auf die Funktion und Wirkung von Vorbildern

6.2.4 Die Rolle von Anti-Vorbildern für die Entwicklung professioneller

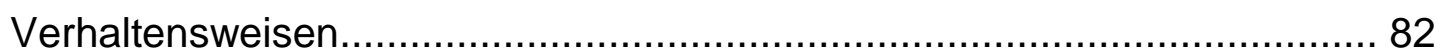

6.2.5 Vorbildwirkung im direkten Kontakt.................................................. 82

6.2.6 Vorbildfunktion prägender Erfahrungen ............................................. 83

6.2.7 Lernen von Kommilitonen: mehr als nur Erfahrungslernen ........................ 84

6.2.8 Kompetenz und Professionalität im Studium erwerben.............................. 85

6.2.9 Mangelnde Rollenbestätigung und Zugehörigkeit ................................. 87

6.3 Stärken und Schwächen der Untersuchung ......................................... 89

6.4 Schlussfolgerungen und Implikationen für die Praxis ........................... 91

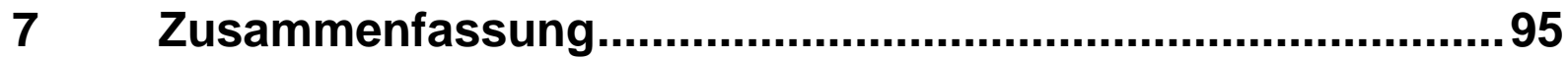

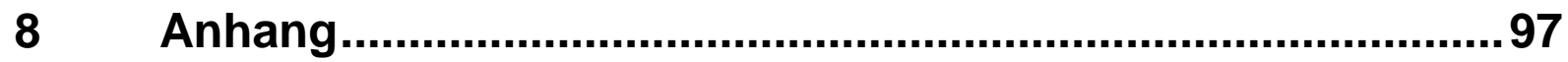

8.1 Information und Aufklärung für Studienteilnehmer/innen der

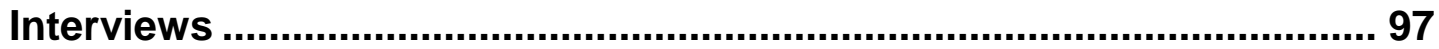

8.2 Liste der Hauptkategorien der ELSAS-Studie mit zugehörigen

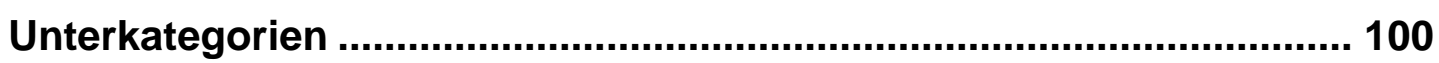

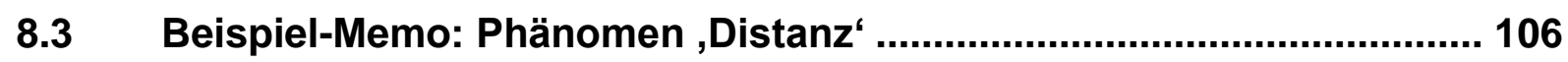

8.4 Zitate des Ergebnisteils im Volltext.................................................... 109

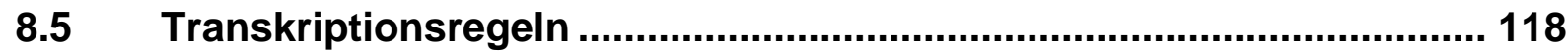

8.6 Reduktionstabelle der Hauptkategorien ........................................... 120

8.7 Beispiel einer Mind-Map zum Phänomen Ideal ................................... 121

$9 \quad$ Literaturverzeichnis .......................................................... 122 


\section{Vorwort}

„Studierende sind hungrig nach positiven Erfahrungen im Medizinstudium.“

Dieser Satz aus einer amerikanischen Studie von Karnieli-Miller (2010) gibt treffend wieder, was auch mein erster Eindruck der für diese Arbeit durchgeführten Interviews war.

Vorbilder können Studierenden zu positiven Erfahrungen im Studium verhelfen. Meine eigene Erfahrung mit Vorbildern ist, dass sie mir Mut für meinen Werdegang als Ärztin machen und mich anspornen, mein Bestes zu geben. Wer ein Vorbild hatte, wird bestätigen können, wie motivierend es ist, eigene Ziele und Wünsche in dem Wirken eines anderen Menschen verwirklicht zu sehen.

In dieser Studie geht es nicht darum, Kritik an denjenigen zu üben, die an der Gestaltung der Curricula medizinischer Hochschulen beteiligt sind. Vielmehr möchte ich das Erleben von Studierenden in den Mittelpunkt stellen. Dem gilt auch mein Forschungsinteresse an Vorbildern und prägenden Personen im Medizinstudium.

Ich wollte wissen, welche Personen für andere Medizinstudierende von Bedeutung waren und welche Themen sie in Bezug auf das Studium beschäftigten, um diese unter dem Gesichtspunkt kollektiver Erfahrungen im Medizinstudium zu analysieren. 


\section{$1 \quad$ Einleitung}

Neue Lehrmethoden sollen die Ausbildung zukünftiger Ärzte verbessern. Dies kann durch ,Technik' geschehen, z.B. durch den Simulator "Harvey"1 oder kommunikativ durch Simulationspatienten. Demgegenüber ist die Idee, Vorbilder in der medizinischen Ausbildung einzusetzen, alles andere als neu. Die Ausbildung von Ärzten erfolgte seit dem fünften Jahrhundert vor Christus in einem Meister-LehrlingsVerhältnis (Wilmanns 2003). Bis zum späten Mittelalter setzte sich das Lernen am Vorbild als gängigste Form der Ausbildung von Ärzten fort (Flexner 1912). Durch die ständige Begleitung zumeist nur eines einzigen Arztes erlernten Kandidaten den Beruf.

Viele Lerninhalte werden in der Medizin - auch heute noch - auf ,implizitem ${ }^{2}$ Weg vermittelt. Dies betrifft vor allem geläufige Umgangsformen und die ,Medizinkultur ${ }^{3}$. Professionelle Verhaltensweisen zu entwickeln, bleibt in Deutschland häufig den Studierenden selbst überlassen ( $\mathrm{vgl}$. Witte 2010). Dabei können alle Kontakte während des Studiums eine prägende ${ }^{4}$ Wirkung auf Studierende haben. Vor diesem Hintergrund überrascht, dass es in Deutschland kaum Forschung über Vorbilder im Medizinstudium und deren Einsatz in der Lehre gibt. Liegt dies daran, dass ihr Einfluss und Potenzial für die Kompetenzen zukünftiger Ärzte unterschätzt wird? Ist dies wiederum in einer naturwissenschaftlich orientierten, materiellen Ausrichtung der Schulmedizin begründet, die eine im Wesentlichen nosologisch und biomedizinisch orientierte Ausbildung (vgl. Herrmann 2011) mit sich bringt? Fest steht, dass es zunächst eines besseren Verständnisses von Vorbildern bedarf sowie der komplexen Zusammenhänge, die mit einer Vorbildwirkung verbunden sind, und einer detaillierteren Beleuchtung der Situationen, in denen Begegnungen stattfinden. Erst dann kann

\footnotetext{
${ }^{1}$ Harvey ist ein lebensgroßer Patientensimulator, der verschiedene Herzerkrankungen und Herztöne nachahmen kann.

${ }^{2}$,Implizit' bezieht sich auf unausgesprochene Wissensinhalte. Der Begriff „Implizites Wissen“ wurde von Polanyi (1985) eingeführt und bezieht sich auf Wissen, das verbal nicht ausdrückbar ist, sich aber im Handeln widerspiegelt und auf diesem Wege vermittelt wird.

${ }^{3}$ Hier wird von einer auf den ,Kulturraum Medizinstudium' eingrenzbaren und für diesen spezifische Kultur ausgegangen, die aus der Tradition medizinischer Praxis hervorgegangen ist und bestimmte Bedingungen vorgibt. Zur Definition von ,Medizinkultur' vgl. Fußnote 23 und 24.

${ }^{4}$ Der Begriff ,prägend' wird in dieser Arbeit für Situationen und Menschen gebraucht, die im weitesten Sinne einen Einfluss auf die Studierenden und ihr Studium hatten. Dabei zählen nicht objektivierbare Faktoren, sondern die subjektive Wahrnehmung der Studierenden.
} 
über den gezielten Einsatz von Vorbildern in der Lehre nachgedacht werden und darüber, wie deren Wirksamkeit auch bei der Gestaltung von Curricula mit einbezogen und genutzt werden könnte.

In der vorliegenden Arbeit geht es zunächst darum, wer überhaupt prägende Personen und Vorbilder für Studierende der Medizin sind. Die Arbeit wird zeigen, dass viele verschiedene Personen und weit über ,klassische Formen des Lernens am Vorbild $^{5}$ hinausgehende Vorbildfunktionen und Einflüsse die heutige ,Medizinkultur’ prägen. Darüber hinaus geht es um Faktoren, die für die Wirkung und Funktion von Vorbildern eine Rolle spielen.

\footnotetext{
${ }^{5} \mathrm{Vgl}$. Bandura et al.(1963). Auf Theorien, die sich mit Imitation beschäftigen, wie das Lernen am Modell oder das Modell der klassischen Konditionierung (vgl. u.a. Allport, Humphrey, Piaget, Pawlow) wird in dieser Arbeit nicht weiter eingegangen.
} 


\section{Stand der Forschung}

Was an neueren Kenntnissen über Rahmenbedingungen der Lehre an medizinischen Fakultäten und Prozesse der Sozialisation von Medizinstudierenden bekannt ist, verdanken wir v.a. amerikanischen Studien. Einer der ersten Forscher, die den Begriff, role model' (Vorbild) im Hinblick auf die Sozialisation von Medizinstudierenden prägte, war Merton (1957). Er stellte die Hypothese auf, dass sich Menschen mit ,Referenzgruppen' sozialer Positionen vergleichen, die sie anstreben. Maßgeblich beeinflusste auch die von Becker et al. (1977) veröffentlichte Studie „Boys in White“ die wissenschaftliche Debatte über kollektive Erfahrungen von Medizinstudierenden. Sie lenkten den Fokus auf das soziale Umfeld von Medizinstudierenden. Im deutschsprachigen Raum gehörten Autoren wie Burkart (1983) und diejenigen des ,Murrhardter Kreises' zu den Ersten, die Interesse an der universitären Sozialisation im Medizinstudium zeigten und Kritik an einer primär am biomedizinischen Krankheitsmodell orientierten Ausbildung von angehenden Ärzten übten (vgl. Arnold 2002). Was Studierende während ihrer Ausbildung außerhalb des regulären Curriculums lernen, wurde lange unterschätzt (Karnieli-Miller et al. 2010). Hafferty und Franks (1994) stellten fest, dass vieles von dem, was im Medizinstudium gelehrt, und das meiste von dem, was gelernt wird, außerhalb des formellen Lehrplans stattfindet. Der Einfluss des so genannten ,geheimen Lehrplans' (hidden curriculum) auf die Professionalisierung der Studierenden und die Entwicklung der ärztlichen Identität spielt dabei eine entscheidende Rolle (Szauter et al. 2009); (Hafferty und Franks 1994). Als ,hidden curriculum wird das Lernen bezeichnet, das im informellen Austausch mit anderen Mitgliedern einer Fakultät geschieht oder als bedeutsame Information en passant vermittelt wird. Bei dieser Vermittlung von Wissen, Einstellungen und Haltungen durch organisatorische, strukturelle und kulturelle Einflüsse in Lehreinrichtungen spielen Vorbilder eine besondere Rolle (Gaufberg et al. 2010). Sie vermitteln über ihr Verhalten bestehende ,Medizinkultur ${ }^{6}$.

Vorbilder im Medizinstudium als Teil des heimlichen Lehrplans und professioneller Sozialisation wurden seit den 80er Jahren in mehreren englischsprachigen Studien untersucht und charakterisiert. Shuval und Adler (1980) kamen zu dem Schluss,

\footnotetext{
${ }^{6}$ Zur Verwendung des Begriffs ,Medizinkultur` vgl. Fußnote 3, 23 und 24.
} 
Stand der Forschung

dass der multidimensionale Prozess des Vorbild-Seins nach einer Typologie verlangt. Ihre Analyse führte zu drei grundlegenden Mustern der Interaktion mit Vorbildern: aktive Identifikation, aktive Ablehnung und passive Orientierung.

Zahlreiche Studien untersuchten Eigenschaften von Vorbildern, ihre Rolle in der medizinischen Lehre und ihre Wirkung auf Medizinstudierende: Nach Wright et al. (1997) sind für die Wahl eines Vorbildes Persönlichkeit, Kompetenz und didaktische Fähigkeiten in der Lehre entscheidend. Wright und Carrese (2002) betonten für die Vorbild-Wirkung die Bedeutung guter klinischer Fähigkeiten und die Tatsache, sich als Lehrende/r der eigenen Vorbildfunktion bewusst zu sein.

Vorbilder wurden zudem als wichtiger Einfluss auf die medizinische Laufbahn von Studierenden bewertet. So beeinflussen sie die Wahl der Spezialisierung (Wright et al. 1997; Ibrahim 2004). Weissmann et al. (2006) fanden in einem qualitativen Forschungsansatz heraus, dass vorbildliches Verhalten die am meisten gebräuchliche Form ist, Werte, Ethik und die menschlichen Dimensionen der Patientenversorgung zu lehren. Dabei nutzte jeder Dozent (der zuvor von den Studierenden als Vorbild bewertet wurde) seine persönlichen, individuellen Lehrstrategien. Gemeinsam war innen, dass sie Selbstreflexion als Grundlage guter Lehre ansahen.

Vorbilder nehmen auch Einfluss auf die Entwicklung einer professionellen Identität (Gaufberg et al. 2010) sowie auf Einstellungen und Verhalten im späteren Berufsleben (Asghari et al. 2011). Darüber hinaus beeinflussen Vorbilder die Lernbedingungen und die Lernatmosphäre im Medizinstudium (Maudsley 2001).

Inzwischen scheint auch ein breiteres öffentliches Interesse an der Thematik zu bestehen: In einem Artikel des Deutschen Ärzteblatts wurde auf das Lernen am Vorbild als eines der zentralen Modelle der medizinischen Ausbildung hingewiesen (Terzioglu et al. 2003). In der Frankfurter Allgemeinen Zeitung wurde berichtet, dass exzellente Ärzte auf die Frage „Wer war Ihr bester Lehrer?" erfreut reagierten und stets von einer entsprechenden Person zu erzählen wussten (Mark 2014). Ähnliches scheint auch für Studierende zu gelten, die zu Erfahrungen im Medizinstudium an der UMG Göttingen befragt wurden (Gágyor et al. 2012). Dreiundachtzig Prozent der Befragten berichteten über Erfahrungen mit Vorbildern.

Dass Beziehungen zu unterschiedlichen Personen in und außerhalb der medizinischen Fakultät während des Studiums einen starken Einfluss auf die ärztliche Ausbildung haben, ist bekannt (Haidet et al. 2006). In Bezug auf die ,Subkultur' des Me- 
dizinstudiums waren Becker et al. (1977) der Ansicht, Verhalten sei ein Produkt der Interaktionen von Medizinstudierenden, vereint in der Konfrontation mit Problemen in medizinischen Hochschulen.

Die Bedeutung von Kommilitonen für Medizinstudierende wurde hinsichtlich verschiedener Aspekte untersucht, kaum jedoch in Bezug auf eine mögliche Vorbildfunktion. Studien zum Thema „Near-Peer-Teaching“ konzentrierten sich v.a. auf den Wert und die Effizienz der Lehre älterer Studierender (Hudson und Tonkin 2008; Lockspeiser et al. 2008; Ten Cate und Durning 2007). Ein anderes Thema war die Stärkung des Selbstbewusstseins und der Motivation von Medizinstudierenden durch Kommilitonen (Ten Cate und Durning 2007) oder die Rolle von Kommilitonen als Informanten, Unterstützer und Vorbilder (Bulte et al. 2007). Weiterhin ging es um den Nutzen von Studierenden in der Auswahl und Rekrutierung neuer Medizinstudierender für eine Fakultät und den Effekt von Kommilitonen, das Interesse der Studierenden an der Medizin und dem Arztberuf zu steigern (Drouin et al. 2006). Gerade was den Kontext und die Bedeutung studentischer Vorbildwirkungen angeht, mangelt es an detaillierteren Betrachtungen.

Die Mehrheit der oben genannten Studien ist, wie Kenny et al. (2003) bemerkten, ,beschreibender Natur'; was sich hinter dem Begriff ,Vorbild' verbirgt, gleicht weiterhin einer „Black Box“. Wer Vorbilder für Medizinstudierende sind und welche Bedeutung sie haben, schon gar nicht aus Exploration erlebter Situationen im Studium, ist in Deutschland bisher wenig untersucht worden. Das ist Thema der vorliegenden Arbeit. 


\section{$3 \quad$ Fragestellung und Ziel}

Um das Phänomen „Vorbild“ im Kontext des Studiums und der Lebenssituation von Studierenden zu verstehen, sollen in dieser Arbeit die Erfahrungen von Studierenden mit Vorbildern und deren Wirkung exploriert werden. Folgende Forschungsfragen standen zunächst im Mittelpunkt:

- Wer sind Vorbilder im Medizinstudium und was vermitteln sie?

- Was zeichnet diese Vorbilder und die Situationen aus, in denen sie den Studierenden begegnen?

Dies sollte zunächst durch eine Sekundärauswertung der ELSAS-Daten ${ }^{7}$ geschehen. Zusätzlich wurden in Form offener Interviews Informationen über konkrete Situationen gesammelt, in denen Menschen für Medizinstudierende zu Vorbildern werden: ob dies beispielsweise in Gesprächen oder vor allem über Beobachtungen geschah, in isolierten Begegnungen oder längerfristigen Beziehungen. Dadurch sollten auch Einsichten in den ,geheimen Lehrplan ${ }^{18}$ (hidden curriculum) in Ausbildungseinrichtungen für Medizinstudierende gewonnen werden. In diesem Sinne lauteten nun die erweiterten Forschungsfragen:

- Was wird neben regulären Lerninhalten im informellen Austausch mit Vorbildern vermittelt?

- Was ist aus der Sicht der Studierenden vorbildliches Verhalten? Welches Verhalten wird wie bewertet?

Im Verlauf des Auswertungsprozesses zeigte sich, dass neben „typischen“ Vorbildern im Medizinstudium auch andere prägende Personen von den Studierenden als wichtig erachtet wurden, die zumindest nicht bewusst als direkte Vorbilder wahrgenommen wurden: vor allem Menschen im engeren Umfeld, wie Freunde und Lernpartner. Daher wurde die Untersuchung nochmals um folgende Forschungsfragen erweitert:

- Welche Personen sind Medizinstudierenden im Rahmen ihres Studiums und für sie persönlich wichtig?

7 „ELSAS" (der Name der Studie) steht für „Erfahrungen mit Lehrenden, Studierenden und anderen Personen während des Studiums".

${ }^{8}$ Zur Begriffserklärung s. Kapitel 2: Stand der Forschung. 
- Welche Bedeutung und Funktion haben diese Personen für Studierende und in welchem Kontext begegnen sie innen?

- Spielen Vorbilder für Medizinstudierende noch eine Rolle?

- Welche Erwartungen haben Studierende an Vorbilder aus unterschiedlichen Personengruppen?

- Wie wird ,Medizinkultur durch prägende Personen und Vorbilder vermittelt? Und welches Bild vom späteren Beruf wird durch sie vermittelt?

- Welchen Einfluss haben Vorbilder und prägende Personen auf Medizinstudierende? Inwiefern tragen sie insbesondere dazu bei, dass Studierende sich mit dem Studium und mit den Rollen identifizieren, die mit dem zukünftigen Beruf verbunden sind?

Zusammengefasst zielt die Untersuchung auf die Funktion und Wirkung von Vorbildern und prägenden Personen für Medizinstudierende und die Situationen bzw. Kontexte, in denen sie bedeutsam werden oder auch umgekehrt: ihre Wirkung verfehlen. 


\section{$4 \quad$ Studiendesign und Methoden}

\subsection{Erster Studienteil: Auswertung der ELSAS-Daten}

Im ersten Teil dieser Arbeit wurde bereits vorliegendes Textmaterial ausgewertet, das im Rahmen der ELSAS-Studie (vgl. Fußnote 7) des Instituts für Allgemeinmedizin der Universitätsmedizin Göttingen im Wintersemester 2008/2009 erhoben wurde (Hilbert 2012). Die Studie ergab, dass unter allen erfragten positiven Erfahrungen der Medizinstudierenden das Erleben einer Vorbildfunktion die häufigste positive Erfahrung war. Die offensichtlich hohe Bedeutung von Vorbildern im Studium veranlasste uns, ein Folgeprojekt zu diesem Thema zu konzipieren (s. 4.25.1.

Zweiter Studienteil: Narrative Interviews), das sich eingehender mit Vorbildern und ihrer Wirkung im Medizinstudium befasst. An der ELSAS-Studie beteiligt waren Ildikó Gágyor und Wolfgang Himmel, die auch die Arbeitsgruppe für diese Dissertation leiteten.

\subsubsection{Studienteilnehmer und Fragebogen der ELSAS-Studie}

An der ELSAS-Studie (Hilbert 2012) nahmen Medizinstudierende aus allen Semestern der UMG teil. Die Vorbildwirkung von Personen war einer von zehn Themenbereichen des Online-Fragebogen. Bejahten die Studierenden Erfahrungen auf einem Gebiet, bestand die Möglichkeit, in einem Freitextfeld Erfahrungen mit eigenen Worten zu beschreiben. Dieses von Nadine Hilbert nicht weiter ausgewertete Textmaterial bot das Datenmaterial für den ersten Teil der vorliegenden Studie.

\subsubsection{Wahl der Auswertungsmethode des ersten Teils}

Zur Auswertung der Freitext-Daten der ELSAS-Studie wurde die zusammenfassende Inhaltsanalyse nach Mayring (2010) gewählt (s.a. Flick et al. 2007). Sie beinhaltet im Wesentlichen eine "Materialreduktion durch Zusammenfassung" (Marx 2009b), die durch Abstraktion ein überschaubares Korpus schafft, das immer noch ein Abbild des Grundmaterials ist (Mayring 2002). Dieses Vorgehen ermöglicht eine systematische, regelgeleitete Textbearbeitung, indem am Material schrittweise Kategorien entwickelt werden. 


\subsubsection{Charakterisierung des Materials}

Die Teilnehmer/innen der ELSAS-Studie beantworten in Freitexten die Frage: „Sind Ihnen im Studium Personen begegnet, die eine Vorbildwirkung auf Sie hatten? Bitte beschreiben Sie nun etwas genauer, durch wen, wo, wann und wie oft bestimmte Personen eine Vorbildwirkung auf Sie hatten. Schildern Sie die Situation, die Ihnen am stärksten in Erinnerung geblieben ist." Die Daten lagen zu Beginn der vorliegenden Arbeit bereits in digitalisierter, schriftlicher Form als Tabelle vor. Es standen insgesamt 200 ausgefüllte Freitextfelder für die Analyse zur Verfügung.

\subsubsection{Zusammenfassende Inhaltsanalyse nach Mayring}

Das methodische Vorgehen orientierte sich an der von Mayring (2010) beschriebenen ,Zusammenfassenden qualitativen Inhaltsanalyse‘ mit folgenden Auswertungsschritten:

\section{Bestimmen von Analyseeinheiten}

,Kodiereinheiten' als Analyseeinheiten definieren, was als minimaler Textbestandteil unter eine Kategorie fällt, und legen das zu analysierende Textmaterial fest. Als Kodiereinheit wurden hier alle Textstellen definiert, die auf die Fragestellung „Was sind Vorbilder und was vermitteln sie?" antworten. Jede Aussage mit vollständigen Angaben über die Person, Erlebnisse, Wirkung und Bewertungen von Vorbildern wurde übernommen, bedeutungslose und -gleiche Paraphrasen wurden gestrichen (Lamnek 2005). Antworten, die unverständlich oder unklar waren und z.B. aufgrund ihrer Kürze (wenige Worte) keine Analyse mit der gewählten Methode zuließen, wurden ausgeschlossen.

\section{Einschlusskriterien}

1. Angaben zur Person von Vorbildern (Position und Fach) und zum Kontext (Ort, Zeit und Situation).

2. Schilderung von Erfahrungen mit Vorbildern, also die Beschreibung von Situationen, die mit innen erlebt wurden, speziell das Verhalten des Vorbilds.

3. Nennung von Eigenschaften der Vorbilder oder Charakterisierungen von Vorbildern. 


\section{Ausschlusskriterien}

1. Wiederholungen.

2. Textteile, in denen unklar blieb, was das Vorbildliche oder die Vorbildfunktion für den/die Studierende/n ausmachte, z.B. „seine Sichtweise auf den Arztberuf“ $(319)^{9}$, wobei nicht gesagt wurde, worin diese genau besteht.

3. Aussagen ohne konkrete Informationen, interpretierbaren Inhalt, (z.B. „eine auBerordentlich gute Famulatur").

4. Argumentationen und Meinungsäußerungen ohne Zusammenhang mit der Forschungsfrage, (z.B. „sind eigentlich alles selbstverständliche Verhaltensweisen, die jedoch nicht immer zu finden sind."). Häufig waren diese auch sprachlich gut erkennbar (,ich finde“ $\left.(52)^{10}\right)$.

\subsubsection{Reduktion des Materials}

Die Reduktion erfolgte in mehreren Schritten. Im Anhang (8.6) findet sich eine ,Reduktionstabelle der Hauptkategorien“ mit Wiedergabe der zur Bildung der Hauptkategorien relevanten Kodiereinheiten und deren Analyseschritte. Ziel war die sprachlich vereinheitlichte Kurzfassung der analytisch relevanten Inhalte.

\section{Paraphrasierung}

Inhaltstragende Teile wurden im Zuge der so genannten Paraphrasierung auf eine einheitliche Sprachebene und in grammatikalische Kurzform gebracht durch Auslassung aller „ausschmückenden“ Textbestandteile (Mayring 2010) und Füllwörter (z.B. eigentlich) und Sonderzeichen. Für eine verständliche Satzstruktur wurden fehlende Satzteile (z.B. Personalpronomen) in eckigen Klammern ergänzt. Während des Paraphrasierungsschrittes wurden zudem Rechtschreib- und Grammatikfehler korrigiert und direkte in indirekte Rede umgewandelt. Bei der namentlichen Erwähnung von Personen wurden der Zusatz „[konkret genannte/r...]“ und die Person oder Funktion z.B. [ranghoher Arzt/Professor] in der Paraphrase aufgeführt.

Unterschiedliche Sinneinheiten, die zumeist zur Aufnahme in unterschiedliche Kategorien führten, wurden bereits auf der Ebene der Paraphrasen getrennt. (Tabelle 1).

\footnotetext{
${ }^{9}$ Die Zahl in der Klammer entspricht der Nummer des Zitates im Originaltext (den von den Studienteilnehmenden in den Fragebögen formulierten Freitexten).

${ }^{10} \mathrm{~S}$. Fußnote 9.
} 


\begin{tabular}{|l|l|}
\hline Zitat & Zugehörige Paraphrasen \\
\hline $\begin{array}{l}\text { „Ich habe einen Arzt kennen gelernt, der nicht nur } \\
\text { fachlich top war, sondern trotz Zeitmangel zu } \\
\text { allen, ob Patient oder Student immer freundlich }\end{array}$ & [Vorbildlich war:] \\
$\begin{array}{l}\text { war. Plus: Souveränität, sich nicht sicher zu sein, } \\
\text { nochmal nachschauen zu müssen.“ }\end{array}$ & $\begin{array}{l}\text { auch trotz Zeitmangel zu allen, ob Patient oder } \\
\text { Student immer freundlich war. }\end{array}$ \\
& $\begin{array}{l}\text { Der Arzt hatte die Souveränität, noch einmal } \\
\text { nachzuschauen, wenn er sich nicht sicher war. }\end{array}$ \\
\hline
\end{tabular}

Tabelle 1: Beispiel für die Aufteilung eines Zitats auf zwei Paraphrasen anhand des Zitates Nr. 138.

\section{Reduktion}

Im Rahmen der Reduktion wurden Textstellen zusammengefasst. Für den ersten Reduktionsschritt wurde ein einheitliches Abstraktionsniveau festgelegt. Dieses wurde so gewählt, dass es weiterhin aussagekräftige Generalisierungen ermöglichte. Formulierungen, die bereits dem festgelegten Abstraktionsniveau entsprachen, wurden in zusammengefasster Form übernommen (s. Zitat 3 in Reduktionstabelle der Hauptkategorien, Anhang 8.6), ebenso Paraphrasen, die über dem festgelegten Abstraktionsniveau lagen. Die Paraphrasen, die unter dem festgelegten Abstraktionsniveau lagen, wurden hingegen auf ein höheres Abstraktionsniveau gebracht (Mayring 2010).

Da es sich bei dem vorliegenden Material um große Textmengen handelte, erfolgte durch Wiederholen dieses Prozesses auf höherem Abstraktionsniveau eine zweite Reduktion (vgl. Anhang Nr. 8.6 Reduktionstabelle der Hauptkategorien).

Umgangssprache, Redewendungen und metaphorische Sprachelemente wurden einem höheren, formalen Sprachniveau angepasst. Angaben zum Ort und zur Situationen wurden ebenfalls in einheitlicher Form verallgemeinert (Ereignisse aus dem Praktischen Jahr, Famulaturen und Untersuchungskurse wurden als „klinische Tätigkeit“" wiedergegeben; Unterricht am Krankenbett und Seminare als "Lehrveranstaltungen“).

\section{Selektion}

Im Rahmen der Selektion wurden inhaltsgleiche Paraphrasen gestrichen (vgl. Mayring 2010) und Aussagen, die weiterhin als zentral oder inhaltstragend erachtet 
Studiendesign und Methoden

wurden, übernommen. Eine weitere Reduktion erfolgte durch Bündelung und Integration. Die Generalisierungen wurden so sortiert, dass inhaltsähnliche Aussagen nebeneinander standen und Paraphrasen mit gleichem oder ähnlichem Gegenstand in eine Aussage integriert wurden.

\section{Kategoriensystem}

Die im vorigen Schritt dargestellte Selektion mit Zusammenfassung inhaltsgleicher oder - ähnlicher Aussagen bildete die Grundlage des Kategoriensystems. Zitate, die zu mehreren Kategorien passten, wurden entweder der inhaltlich überwiegenden Kategorie oder mehreren Kategorien zugeordnet. Ein Beispiel ist die Aufteilung des Zitates Nr. 285 (Tabelle 2). Das Zitat beinhaltet Aussagen zu drei unterschiedlichen Themen. Da keine Aussage überwog, wurden die unterschiedlichen Teile drei verschiedenen Kategorien zugeordnet.

\begin{tabular}{|c|c|}
\hline Zitat & Kategorie \\
\hline $\begin{array}{l}\text { Ein Arzt, der mit uns den Untersuchungskurs } \\
\text { Chirurgie gemacht hat [...]. Der Arzt hat es ver- } \\
\text { standen, eine angenehme Situation zu schaffen, } \\
\text { auch wenn Patienten dabei waren und wir viele } \\
\text { Studenten waren. }\end{array}$ & $\begin{array}{l}\text { Eingehen auf Bedürfnisse der Studierenden und } \\
\text { Unterstützung }\end{array}$ \\
\hline Er hat Ruhe und Souveränität ausgestrahlt. & $\begin{array}{l}\text { Selbstbewusste und kompetente Wirkung durch } \\
\text { Auftreten und Verhaltensweisen von Ärzten }\end{array}$ \\
\hline $\begin{array}{l}\text { Auch gab es schon Vorlesungen, v.a. in den Mo- } \\
\text { dulen perioperatives Management und Mikrobio- } \\
\text { logie, die mich einfach mitgerissen haben, weil } \\
\text { die Dozenten für ihr Fach zu leben scheinen und } \\
\text { absolut die Faszination rüberbringen können. Es } \\
\text { ist so schön, wenn man am Ende einer Vorlesung } \\
\text { überrascht ist, dass sie schon vorbei ist. Toll ist } \\
\text { es auch, wenn die Dozenten ganz offensichtlich } \\
\text { gerne erklären und lehren. }\end{array}$ & $\begin{array}{l}\text { Ärzte, die in Lehrsituationen Studierende für den } \\
\text { Beruf begeistern }\end{array}$ \\
\hline
\end{tabular}

Tabelle 2: Zuordnung von Zitaten zu unterschiedlichen Kategorien am Beispiel des Zitates Nr. 285.

Kurze Aufzählungen von Eigenschaften in Zitaten erschwerten die Kategorienbildung. Die Zuordnung wurde dann nach der zentralen Aussage entschieden (Marx 
Studiendesign und Methoden

2009a). Sich überschneidende Kategorien wurden gestrichen bzw. in neue überführt. Hauptkategorien wurden dann durch erneute Zusammenfassung gebildet. Im Verlauf der Analyse wurden die Hauptkategorien mehrfach revidiert und überarbeitet (das Abstraktionsniveau musste z.B. mehrfach angepasst werden, da es anfänglich zu hoch war). Am Ende dieses Prozesses stand ein „neues, allgemeineres und knapperes Kategoriensystem“, das wiederum überprüft wurde (Mayring 2010). Die endgültigen Hauptkategorien finden sich in Ergebniskapitel 5.1.

\subsection{Zweiter Studienteil: Narrative Interviews}

\subsubsection{Begründung für die Ausweitung der Studie}

Das Material der ELSAS-Studie gab inhaltlich zwar Einblicke in das, was die Studierenden als vorbildlich erleben, es blieb jedoch bei abstrakten Nennungen von Personen und deren Eigenschaften (vgl. Kapitel 4.1.3: Charakterisierung des Materials). Auch bot das knappe Datenmaterial keine detaillierte Einsicht in konkrete Situationen, die eine „Interpretation tiefer liegender Sinn- und Bedeutungszuschreibungen“ (Marx 2009a) erlaubt. Zusätzliche Daten aus Interviews sollten helfen, genauere Erkenntnisse über die Umstände zu gewinnen, in denen Studierende vorbildliches Verhalten beobachteten.

Die ,Inhaltsanalyse nach Mayring' sollte durch eine ergebnisoffenere Methodik ergänzt werden, die an die Grounded Theory angelehnt ist. Die Analyse erfolgt bei Mayring eng am Text, auf einer am Wortlaut der Daten orientierten Ebene (Marx 2009b). Die Grounded Theory, als analytisch interpretatives Verfahren hingegen, rekonstruiert aus den Daten die Interpretationen der Handelnden selbst (Strauss und Corbin 1996). Da in dieser Studie die Analyse von individuellen Erfahrungen, Wahrnehmungen, Verarbeitung und Bedeutungszuschreibung von Studierenden im Vordergrund stehen sollte, erschien diese Auswertungsmethode am geeignetsten.

Wie bei qualitativen Studien üblich, wurde im Verlauf des Auswertungsprozesses die Forschungsfrage überarbeitet. So wurde unter Berücksichtigung erster Ergebnisse das Forschungsinteresse auf prägende Personen erweitert (s. Kapitel 3: Fragestellung und Ziel). 


\subsubsection{Narratives Interview}

Das Narrative Interview schien für den zweiten Teil der Studie als methodischer Zugang geeignet, da es den Interviewten die Möglichkeit gibt, ihre Erzählung nach eigenem Ermessen zu strukturieren und ihre Wirklichkeitsdefinitionen dem Forscher mitzuteilen (Lamnek 1989). Das Narrative Interview hat gegenüber standardisierten Methoden (z.B. mit Leitfaden) den Vorteil, keine Antworten vorzugeben. Es besteht aus zwei Phasen: Die erste Phase beginnt mit der Erzählaufforderung (s. 4.2.4: Durchführung der Interviews), worauf bestenfalls eine längere freie Erzählung folgt. Durch die Erzählaufforderung wird versucht, eine Erzählung zu generieren, die in ihrer Struktur den Orientierungsmustern und dem Handeln der befragten Person am nächsten kommt (Bohnsack et al. 2003). Die zweite Phase bildet der Nachfrageteil, in dem ,interne Nachfragen' zum Gesagten und zusätzliche für das Forschungsthema relevante ,externe Nachfragen' gestellt werden. Besonders hierfür konnten die Ergebnisse der Zwischenauswertung der ELSAS-Studie für die Gestaltung der Interviews genutzt werden.

\subsubsection{Rekrutierung und Vorbereitung der Studienteilnehmer/innen}

Voraussetzung zur Teilnahme an den Interviews war die Immatrikulation im Studienfach Humanmedizin an der UMG Göttingen. Die Studienteilnehmerinnen und Studienteilnehmer wurden über Dritte vermittelt rekrutiert ${ }^{11}$, um ein zu enges Verhältnis in der Interviewsituation zu vermeiden. Die Studierenden wurden dann persönlich oder per Telefon über das Forschungsvorhaben und den Ablauf der Interviews informiert (siehe Anhang 8.1). Eine zu weitgehende Präzisierung des Forschungsthemas wurde vermieden, um eine Vorbereitung auf Fragen zu verhindern und eine spontane Erzählung zu ermöglichen.

${ }^{11}$ Damit ist gemeint, dass keine Personen aus dem direkten Freundeskreis für die Studie befragt wurden. „Über Dritte vermittelt" bedeutet, dass der Kontakt zu den Personen, die an der Studie teilnahmen über Bekannte (Kommilitonen, zu denen z.B. über Lehrveranstaltungen ein Kontakt bestand oder Studierende, zu denen der Kontakt über andere bereits interviewte Personen) hergestellt wurde. 


\section{Sample}

Um eine möglichst große Bandbreite an Studienerfahrungen zu erhalten, wurden Studienteilnehmer und -teilnehmerinnen zufällig ausgewählt, unter Berücksichtigung des Semesters und Geschlechts.

Aufgrund des zweizeitigen Vorgehens (die ELSAS-Studie wurde im Jahr 2008; die Interviews wurden 2012 durchgeführt), gab es im Sample keine Überschneidung. Im Sinne von Esser (1986) wird jedoch davon ausgegangen, dass Studierende als ,Agenten von fixen Rollenerwartungen' die Frage nach Vorbildern als soziales Phänomen auch dann ,repräsentativ' beantworten können, wenn die Gruppe kleiner ist und es sich um andere Personen handelt als in der ursprünglichen Befragung.

\subsubsection{Durchführung der Interviews}

Insgesamt wurden 15 Interviews durchgeführt, aufgezeichnet und anschließend transkribiert. Das Interview war in seinem Ablauf durch die beiden Teile - Erzählaufforderung mit Einstiegsfrage und Nachfrageteil - festgelegt und wurde wie folgt eingeleitet:

„Ich hatte Dir ja schon gesagt, dass ich mich für Medizinstudierende und ihre Erfahrungen interessiere, die sie im Medizinstudium gemacht haben mit Menschen, die wichtig waren für sie und für ihre Ausbildung - mich interessieren alle Erlebnisse und Situationen, die Dir einfallen, z.B. Gespräche oder einfach Begegnungen auf Station, während der Pause in der KaffeeEcke, im Hörsaal, während Tutorien oder in der Lerngruppe. Ich würde Dich bitten, an den Beginn Deines Studiums zurückzudenken, welche Begegnung fällt Dir ein? Und Du erzählst dann davon, wie es dann weiterging am besten bis heute."

Die dann einsetzende Erzählung wurde nicht durch Fragen seitens der Interviewerin unterbrochen. Zum Abschluss des ersten Blockes wurden Ergänzungen zur bisherigen Erzählung erbeten. Das Erzählte wurde für den nun folgenden Nachfrageteil durch Notizen festgehalten. Die Nachfragen orientierten sich an den Inhalten der bisherigen Erzählung. Mit Hilfe der Notizen wurden interne Nachfragen formuliert, die detaillierter auf angesprochene Themenbereiche oder erwähnte Personen eingingen. Auch die Rückfragen wurden erzählgenerierend gestaltet unter Verzicht auf Suggestivfragen. Hier zwei Beispiele für den Typus von Nachfragen:

- „Du hast erwähnt, dass... Könntest Du darüber noch mehr erzählen?“

- „Kannst Du mir diese Situation/diese Person noch einmal genauer beschreiben?“ 
Studiendesign und Methoden

Externe Nachfragen sollten sicherstellen, dass bestimmte Themengebiete angesprochen werden. Wenn unklar blieb, ob Personen als Vorbilder angesehen wurden, wurde z.B. gefragt:

- „Andere Studierende haben erzählt, dass sie im Studium auch einige Vorbilder hatten. Fallen Dir auch Situationen mit Personen ein, die für Dich Vorbilder waren?“

Ablauf und Aufnahme der Interviews wurden nur dann unterbrochen, wenn die Studienteilnehmer eine Pause wünschten.

\subsubsection{Auswertung}

\section{Charakterisierung des Materials}

Das Datenmaterial besteht aus 15 Narrativen Interviews, die von Juni bis August 2012 geführt wurden und zwischen 23 und 85 Minuten lang sind. Die Audiodateien und die zugehörigen Transkripte werden sicher verwahrt. Die für den Ergebnisteil verwendeten Zitate finden sich im Volltext im Anhang 8.4; eine Liste der Transkriptionsregeln im Anhang 8.5. Die Auswertung der Interviews erfolgte mit Unterstützung des Computerprogramms Atlas.ti. Diese Software - im Sinne einer Computer Assisted Qualitative Data Analysis (CAQUDAS) - ist geeignet, größere Datenmengen analytisch zu bearbeiten und die einzelnen Auswertungsschritte zu systematisieren.

\subsubsection{Durchführung der Auswertung}

Die Analyse erfolgte als fortlaufender Prozess „im ständigen Wechsel“ (vgl. Marx 2009b).

\section{Offenes Kodieren}

,Kodieren' meint das Benennen von Sinneinheiten oder Phänomenen anhand der vorliegenden Daten. Corbin und Strauss beschrieben dieses Verfahren als „die kreative Erarbeitung von Begriffen" für allgemeine, in den Daten enthaltene Phänomene (vgl. Strauss und Corbin 1996). Im Rahmen des offenen Kodierens werden „die Daten in einzelne Teile aufgebrochen, gründlich untersucht [und] auf Ähnlichkeiten und Unterschiede hin verglichen“ (Strauss und Corbin 1996). Begriffe für die theoretischen Codes wurden teilweise in den Äußerungen der Informanten selbst gefunden, die dann als „In-vivo-Codes“ (Strauss und Corbin 1996) bezeichnet werden (Abbildung 1). 
P: Äh ... also es ist eigentlich so dass ich ähm als ich hier angefangen habe nicht so viele Leute kannte ähm ich hab erst mal mit also erst mal Kontakte mit Leuten aus //l: $\mathrm{mhm} / /$ also aus meinem Heimatland verknüpft und ja das war halt ganz wichtig für mich weil das hat mir ein (Gefühl) von Zugehörigkeit //l: $\mathrm{mhm} / /$ genau beigebracht und ich meine es ist ja so dass man ja in einem Land ist wo man erst mal ganz allein ist ich hab zwar Cousins hier aber ähm die sind in anderen Städten //l: $\mathrm{mhm} / /$ und ähm das war halt so erst mal ganz wichtig für mich dass ich Leute hier getroffen habe mit denen ich mich ja gleich richtig verstanden habe //l: $\mathrm{mhm} / /$ Leute die kulturell äh ja genau so drauf sind wie ich und so //l: $\mathrm{mhm} / /$ und ich glaube das war halt oder das sind (wichtige Begegnungen) die ich bis jetzt gehabt hab weil bis heute sind noch die Kontakte eben gut auch wenn andere nicht mehr Medizin studieren $/ / \mathrm{l}: \mathrm{mhm} / /$ aber das ist halt wie soll ich sagen trotzdem ja wichtig für mich //I: $\mathrm{mhm} / /$ genau und ja .. \#00:02:23-9\#

ähm meine Freundin auch (X- Name der Partnerin) aber ich hab die erst in der Klinik kennen gelernt //l: $\mathrm{mhm} / /$ das heißt die Zeit äh in der Vorklinik hat hat sie äh kein

Einfluss drauf gehabt und ähm (4) na ich würd mal sagen das sind halt die wichtigsten Leute //: $\mathrm{mhm} / /$ soll ich auch die Namen geben oder \#00:02:53-0\#
2.: Zu Beginn des Studiums nach sozialem Rückhalt und 'Zugehōrigkeit'

E Zugehorigkeit

Abbildung 1: Textstelle S10 003 als Beispiel eines In-vivo-Codes.

Die offenen Codes waren vorläufig und stellten mögliche Lesearten der Textstellen dar. Ziel war die konzeptuelle bzw. theoretische Eröffnung eines Raums möglicher Deutungen eines Phänomens.

\section{Benennen von Phänomenen und Konzeptualisieren}

Die Suche nach Phänomenen im betreffenden Text wird auch als „Konzeptualisieren“ bezeichnet und dient dem Zweck, analytisch Konzepte zu entwickeln (Rosenthal 2005). Nachdem die Interviews offen kodiert waren, wurde ein Auszug aller bisher gefundenen offenen Codes im Gesamten betrachtet: Die Codes wurden untereinander verglichen und nach ,Überthemen' gruppiert. Daraus wurden Phänomene ersichtlich, die in Form von Memos (s.u.) beschrieben wurden. Bei der Analyse weiterer Interviews wurden die bereits erarbeiteten Hypothesen, Codes und Memos (s.u.: Axiales Kodieren und Paradigmatisches Modell) bedacht und neue Textstellen mit den bereits kodierten Daten verglichen, auf neue Phänomene untersucht und kategorisiert (Strauss und Corbin 1996). Anschließend wurden alle gefundenen Phänomene

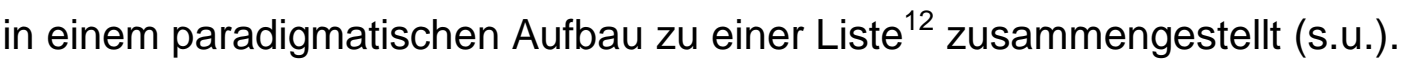

\footnotetext{
12 Im Folgenden als „Phänomen-Liste“ bezeichnet.
} 


\section{Axiales Kodieren und Paradigmatisches Modell}

Im Zuge des axialen Kodierens wurden die Daten „auf neue Art zusammengesetzt“ (Strauss und Corbin 1996). Dies geschah unter Einsatz des Kodier-Paradigmas ${ }^{13}$. Jedem gefundenen Phänomen wurden dabei offene Codes in Form von ursächlichen und intervenierenden Bedingungen, Kontexten, Handlungsstrategien und Konsequenzen zugeordnet (vgl. Bohnsack et al. 2003): Der ,Kontext' beinhaltet die spezifischen Eigenschaften, die zu einem Phänomen gehören (Strauss und Corbin 1996). ,Ursächliche Bedingungen“ sind „Ereignisse [...], die zum Auftreten oder der Entwicklung eines Phänomens führen" (Strauss und Corbin 1996). Intervenierende Bedingungen stellen strukturelle Bedingungen dar, die auf interaktionale Strategien einwirken oder einen intervenierenden Einfluss auf das Phänomen nehmen. ,Handlungsstrategien' beziehen sich auf Inhalte, die dazu dienen, Bedingungen zu erfüllen, um das spezifische Phänomen zu bewältigen oder zu ermöglichen. „Konsequenzen“ werden „Ergebnisse oder Resultate von Handlung und Interaktion“ genannt (Strauss und Corbin 1996). ,Das Phänomen' als zentrale Idee, auf die sich die bisher beschriebenen Bestandteile des Kodier-Paradigmas beziehen, ist eine Einheit von „Beobachtungen“, die konzeptuell zueinander passen (Strauss und Corbin 1996). Abbildung 2 ist ein Beispiel für ein Kodier-Paradigma zum Phänomen „Distanz zu Lehrenden ${ }^{14 ،}$. Jeweils am Ende der Beschreibung einer Hauptkategorie im Ergebniskapitel werden die Ergebnisse in einer Abbildung im Sinne des Kodier-Paradigmas zusammengefasst.

Im Zuge des axialen Kodierens wurden die Phänomene so gruppiert, dass sie einer (Über-)Kategorie ${ }^{15}$ zugeordnet wurden. Anschließend erfolgte eine Überarbeitung der Hypothesen und Kategorien, wobei diese wiederholt anhand der Daten überprüft, teilweise verworfen, weiterentwickelt und kombiniert wurden. Vorläufig erstellte Hypo-

\footnotetext{
${ }^{13}$ Gleichbedeutend mit „Paradigmatisches Modell“.

${ }^{14}$ Zu den Überschriften des Kodier-Paradigmas wurden passende Zitate gesucht. Um den Ursprung des Zitates nachvollziehbar zu machen, wurden sie (wie im Kapitel 4.3: Datenschutz und Genehmigung durch die Ethikkommission erklärt) mit zwei Zahlen versehen. Die erste Zahl hinter dem S (das für Studienteilnehmer/in steht) bezieht sich auf das Interview, die zweite Zahl auf die Textstelle im Transkript.

${ }^{15}$ Der Begriff ,Überkategorie‘ ist hier im Sinne eines Überthemas gemeint, das für mehrere Phänomene gefunden wurde.
} 
Studiendesign und Methoden

thesen ${ }^{16}$ und Konzepte mussten überprüft werden, indem beispielsweise mehrere Textstellen miteinander verglichen und nach Gegenbeispielen gesucht wurden. Konnten Hypothesen nicht in den Daten bestätigt werden, wurden sie verworfen.

16 „Hypothesen" sind hier in Anlehnung an die Grounded Theory als die unterschiedlichen Lesarten einer Textstelle verstanden, die es während der Analyse in Bezug auf das gesamte Textmaterial zu überprüfen gilt (vgl. Strauss und Corbin 1996). 
Phänomen „Distanz zu Lehrenden“

Kontext: Studierende haben wenig Kontakt zu Lehrenden (S12 04)

Ursächliche Bedingung:

1. Dozenten zeigen wenig Interesse an den Studierenden (S08 10)

2. Distanz scheint als professionelle Grundhaltung von Dozenten Studenten gegenüber gesehen zu werden (S04 007, S03 31)

3. Die große Zahl der Studierenden schafft ein Gefühl von Anonymität (S12 04, S03 63)

4. Die Studierenden fühlen sich von Dozenten eingeschüchtert (S12 04)

Intervenierende Bedingung für Minderung der Distanz zwischen Studierenden und Dozenten:

1. Trotz großer Zahl an Studierenden Interesse von Dozenten am Einzelnen (S05 003)

2. Gezielte und engagierte Kontaktaufnahme mit Professoren durch Studierende

Strategie: Offenheit in Interaktionen mit Studierenden identifiziert (S01 04); Dozenten gezielt zur Kontaktaufnahme auswählen, die in vergangenen Interaktionen einen offenen und interessierten Eindruck gemacht hatten.

\section{Konsequenz:}

1. Wunsch nach mehr persönlichen Kontakten zu Dozenten (S07 04, S03 63 und 97)

2. Studierende schreiben selbst kurzen Interaktionen mit Dozenten große Bedeutung zu (S16 23)

3. Vermehrte Suche nach Unterstützung von Kommilitonen (S12 03)

4. Wenig Kenntnis des Dozenten als Person (S01 04)

5. Unsicherheit darüber, ob Dozenten Vorbilder sind, wenn kein persönlicher Kontakt besteht (S07 50)

6. Übergeordnete Konsequenz: Zum Definieren oder Ansehen eines Dozenten als Vorbild zählen für Studierende die Art der Interaktion und ihre Persönlichkeit

Abbildung 2: Beispiel-Kodier-Paradigma zu dem Phänomen ,Distanz zu Lehrenden. 


\section{Memos}

"Memos" sind theoretische Überlegungen in Form von Gedankenprotokollen, in denen Erkenntnisschritte des Analyseprozesses festgehalten werden (Marx 2009b). Während des gesamten Prozesses wurden alle Ideen und Hypothesen, die in der Auseinandersetzung mit den Daten formuliert wurden, in Form solcher Memos festgehalten (Strauss und Corbin 1996). Dabei kann zwischen verschiedenen Typen von Memos unterschieden werden:

- Fallmemos

- Memos zur Vorgehensweise

- Memos zu einzelnen Wörtern ${ }^{17}$ und Codes

- Memos zu Vergleichen zwischen Codes oder Fällen

- Memos zu Kategorien und Kernkategorien

Als Beispiel findet sich im Anhang 8.3 ein Memo zum Phänomen ,Distanz'.

\section{Selektives Kodieren}

Durch ,selektives Kodieren' erfolgte die Integration aller bisher durch axiales und offenes Kodieren erarbeiteten Konzepten und Kategorien in Hauptkategorien. Im Rahmen der Selektion wurden die Codes erneut sortiert, gruppiert und (z.B. doppelte Codes) gestrichen. Um dabei wesentliche Inhalte nicht zu verlieren, wurden Fragen gestellt wie „Was ist in diesem Untersuchungsbereich am auffälligsten“ oder "Was ist der rote Faden der in den Daten enthaltenden Geschichte?", (vgl. Strauss und Corbin 1996). Diese Überlegungen wurden ebenfalls in Form von Memos und Mind-Maps festgehalten (vgl. Anhang 8.7).

\section{Kategorienbildung}

Zur Bildung der Hauptkategorien wurde die (unter Axiales Kodieren und Paradigmatisches Modell) beschriebene Phänomen-Liste erneut mit dem Fokus auf der Suche nach Überthemen durchgegangen. Die entdeckten zentralen Phänomene wurden dann wiederum mit Hilfe des Kodier-Paradigmas ausführlich beschrieben. Das Ergebnis sind die einzelnen Kapitel des Ergebnisteils (die zentralen Phänomene werden in den Überschriften benannt).

\footnotetext{
${ }^{17}$ Vergleichbar mit In-vivo-Codes.
} 


\subsection{Datenschutz und Genehmigung durch die Ethikkommission}

Wie im Kapitel 4 beschrieben, besteht die vorliegende Arbeit methodisch und inhaltlich aus zwei Teilen. Im ersten Teil wurde vorliegendes Textmaterial der ELSASStudie ausgewertet (s. Kapitel 4.1); für diesen Teil lag bereits eine Bewilligung durch die Ethikkommission der Universitätsmedizin Göttingen vor (Antragsnummer 1/6/08). Die Durchführung der Interviews für den zweiten Teil erfolgte nach Prüfung des Ethikantrags durch die Ethikkommission der Universitätsmedizin Göttingen (Antragnummer 28/7/12). Dieser Teil des Forschungsprojektes wurde als nicht beratungspflichtig bewertet.

Bei der Aufzeichnung auf Tonband wurde auf eine Nennung der Namen der Interviewten verzichtet. Auch die Auswertung erfolgte anonym. Die im Zuge der Datenerhebung erstellten Code-Listen mit den Namen der Studienteilnehmenden wurde getrennt von den Transkripten archiviert. Die Namen der Studierenden wurden durch Nummern (z.B. S04) oder der Lesbarkeit wegen durch Pseudonyme in den transkribierten Interviews ersetzt. In der Auswertung wurden keine Namen von Ärzten, Einrichtungen, Städten, anderen Personen etc. übernommen. Die Audiodateien mit den Interviews werden in der Abteilung Allgemeinmedizin archiviert. Die Weitergabe an Dritte ist strikt untersagt.

Das Einverständnis der Studierenden wurde vor der Durchführung der Audioaufzeichnung des Interviews durch eine Einverständniserklärung eingeholt und die Studienteilnehmer/innen über Datenschutzregelungen informiert. Die Studierenden erhielten außerdem ein Informationsblatt (siehe Anhang 8.1), in dem der Forschungsgegenstand erklärt wurde. Dabei wurde insbesondere deutlich gemacht:

- Das Interview ist mit keinerlei Risiko verbunden, die potentiellen Teilnehmer wurden schriftlich zur Teilnahme eingeladen und in einem persönlichen Vorgespräch über die Durchführung der Studie informiert.

- Das Interview wird erst nach Vorlage des „informed consent“ durchgeführt.

- Es erfolgte eine Pseudonymisierung der Transkripte, sodass kein Bezug zur interviewten Person, zu genannten Personen, Orten oder Kliniken hergestellt werden kann.

- Die Audioaufnahmen werden zehn Jahre in einem verschlossenen Schrank mit beschränktem Zugang gesichert aufbewahrt. 
- Bei Widerruf der Zustimmung würden die Daten sofort und endgültig gelöscht werden. 


\section{$5 \quad$ Ergebnisse}

Die Befragung der ELSAS-Studie fand zwischen Dezember 2008 bis April 2009 statt. Zunächst folgen in Kurzform die Ergebnisse, soweit sie in der Vorgängerarbeit (Hilbert 2012; Gágyor 2012) bereits veröffentlicht wurden und das Thema „Vorbild“ berühren: Von 1.222 Studierenden, denen Onlinefragebögen zugesandt wurden, füllten sie 391 vollständig aus. Die „Vorbildfunktion“ war unter allen in der Studie erfragten Erfahrungen mit 83,4\% die häufigste. Stationsärzte $(25,3 \%)$ und Professoren $(23,6 \%)$ waren die am häufigsten genannten Vorbilder und die Personen, von denen vornehmlich Wertschätzung ausging. Die Studienteilnehmer/innen nannten konkrete Personen häufig mit Namen, Dienstrang oder akademische Titel und Funktion (z.B. Chefarzt oder Professor). Neben Ärzten waren weitere Personengruppen Pflegekräfte und Kommilitonen.

Der erste Schritt in der vorliegenden Studie war die Durchsicht und Ordnung des Materials in Hauptkategorien hinsichtlich der Frage „Wer ist Vorbild für Medizinstudierende und was vermitteln diese Personen?" (s. Kap. 5.1).

\subsection{Hauptkategorien der ELSAS-Studie}

Die Analyse der ELSAS-Daten zum Thema „Vorbild" im Rahmen des ersten Teiles dieser Studie zeigte vor allem die Bedeutung von Vorbildern in Bezug auf die Vermittlung von Lerninhalten und das Erteilen von Ratschlägen. Hinsichtlich der Funktion von Vorbildern wurden v.a. ärztliche Eigenschaften geschildert, die die Studierenden als erstrebenswert beschrieben wie ihre professionelle Arbeitsweise. Dazu gehörte z.B. ein gutes Zeitmanagement. Daneben wurde auch von Einstellungen und Haltungen zur Medizin berichtet, die als vorbildlich erachtet wurden wie z.B. „ein wissenschaftlicher Anspruch“. Das Material konnte zu 15 Hauptkategorien (s. Anhang 8.2) zugeordnet werden, die zusammenfassen, welche Personen für die Befragten als Vorbilder gesehen werden:

1. Ärzte, die in herausfordernden Berufssituationen professionell handeln

2. Ärzte mit großem Fachwissen und Kompetenz bei praktischen Tätigkeiten

3. Ärzte, die neben fachlicher Kompetenz als Person besondere Eigenschaften haben

4. Ärzte, die im professionellen Kontext selbstbewusst und kompetent auftreten 
5. Ärzte mit sozialen Kompetenzen im Umgang mit Patienten

6. Pflegekräfte mit Bereitschaft, unter schwierigen Arbeitsbedingungen gute Arbeit zu leisten

7. Ärzte, die einen hohen Anspruch an den Beruf vermitteln

8. Ärzte, die Studierende wertschätzen und unterstützen

9. Ärzte, die die Ausbildung der Studierenden ernst nehmen

10. Ärzte, die in Lehrsituationen Studierende für den Beruf begeistern

11. Ärzte, die Führungskompetenz und Kollegialität vereinen

12. Ärzte, die ethische Grundprinzipien medizinischen Handelns implizit vermitteln

13. Ärzte, die in der medizinischen Hierarchie oben stehen

14. Ärzte, die trotz ihres hohen Ranges Interesse an Studierenden haben und durch ihre Persönlichkeit Vorbilder sind

15. Kommilitonen, die engagiert, kompetent und unterstützend sind.

Im Folgenden soll detaillierter auf die Inhalte der durch Auswertung der OnlineFragebögen gefundenen Hauptkategorien eingegangen werden: Die unter der ersten Hauptkategorie („Ärzte, die in herausfordernden Berufssituationen professionell handeln“) zusammengefassten Eigenschaften und Fähigkeiten bezogen die Studierenden v.a. auf das Verhalten von Ärzten in herausfordernden Situationen wie z.B. Notfallsituationen, in denen sie unter Stress Ruhe bewahrten. Dazu zählten die Studierenden auch, wenn Ärzte unter hoher Arbeitsbelastung Zeit und Geduld für Gespräche aufbrachten, die für Patienten bedeutungsvoll waren (wie das Mitteilen einer schwerwiegenden Diagnose). Im Hinblick auf Arbeitsbelastungen war für die Studierenden zudem ein effektives Zeitmanagement vorbildlich: z.B. bei der Handhabung von mehreren Tätigkeiten zur gleichen Zeit (Multitasking) oder die Bewältigung unterschiedlicher Anforderungen wie die Ausbildung von Studierenden und die Stationsarbeit.

„Fachwissen und Kompetenz" wurden ebenfalls häufig genannt und wurden als wichtige Attribute „professionellen Verhaltens“ von Ärzten erachtet. „Ärzte mit großem Fachwissen und Kompetenz bei praktischen Tätigkeiten" bezog sich auf vorbildlich effektives Arbeiten in der Klinik oder gutes Allgemeinwissen.

Die Studierenden betonten, dass für die Vorbildfunktion von Ärzten gleichzeitig fachliche und persönliche Kompetenzen wichtig seien - ausgedrückt in der Hauptkatego- 
Ergebnisse

rie „Ärzte, die neben fachlicher Kompetenz als Personen besondere Eigenschaften aufweisen". Das sind Vorbilder, die unterschiedliche Kompetenzen in sich vereinen, wobei persönliche Eigenschaften (wie „Bescheidenheit, Freundlichkeit, Höflichkeit, Geduld und Herzlichkeit“) den Studierenden besonders wichtig waren.

Die Hauptkategorie „Ärzte, die selbstbewusst und kompetent auftreten“ drückt aus, dass die Studierenden unter „Professionalität“ auch ein bestimmtes ärztliches Auftreten verstanden. Als Arzt ruhig, kompetent und selbstsicher zu wirken, schien dabei wesentlich zu sein. Bei ranghohen Ärztinnen wurde ein „taffes und kompetentes“ Auftreten für vorbildlich gehalten.

Die Hauptkategorie „Ärzte mit sozialen Kompetenzen im Umgang mit Patienten“ bezieht sich auf die häufige Nennung von Fähigkeiten für einen vorbildlichen Patientenumgang. Diesen beschrieben Studierende im Wesentlichen als „respektvoll und rücksichtsvoll“. Wichtige Eigenschaften waren dabei auch „Feingefühl“ und ein „Geschick" im Umgang mit schwierigen Patienten.

Eine Besonderheit ist die Hauptkategorie 6 „Bereitschaft, unter schwierigen Arbeitsbedingungen gute Arbeit zu leisten"; sie bezieht sich auf einen Einzelfall, in dem das Pflegepersonal als Personengruppe genannt und deren Professionalität als vorbildlich beschrieben wurde.

Die nächste Hauptkategorie „Ärzte, die einen hohen Anspruch an den Beruf vermitteln" schloss sowohl Charaktereigenschaften wie "Gewissenhaftigkeit" als auch Einstellungen zum Beruf wie einen „wissenschaftlichen Anspruch“ ein.

Da bei der Bewertung von Ärzten als Vorbilder auch deren Umgang mit Studierenden eine Rolle spielte, wurde die Hauptkategorie „Ärzte, die Studierende wertschätzen und unterstützen" gebildet. Im Umgang mit Studierenden zählten v.a. ein faires, gleichberechtigtes und nicht hierarchisches Verhalten und Freundlichkeit. Entscheidend für ein vorbildliches Verhalten Studierenden gegenüber war auch eine gute Betreuung in Lehrveranstaltungen, Studierende dabei wertzuschätzen, auf den Einzelnen einzugehen und Selbstvertrauen und Selbstständigkeit zu fördern:

„Der Dozent hat die Studenten als Person wahrgenommen. Mit provokativen Fragen erreicht, dass man zum Nachdenken kommt." (134) ${ }^{18}$

\footnotetext{
${ }^{18}$ Nummer des Zitates (vgl. Fußnote 9).
} 
Ergebnisse

„Im PJ hatte ich eine enge Betreuung durch meinen Aufenthalt in der Allgemeinmedizin. Da diese sehr gut war hatte ich die Möglichkeit sehr viel theoretisch aber vor allem praktisch und in der Gesprächsführung zu lernen. Ein Oberarzt in X-(Name eines Ortes) hat unserer Blockpraktikanten-Gruppe das Erheben eines Neurologischen Status richtig ausführlich beigebracht. Er hat jeden die einzelnen Untersuchungen persönlich durchführen lassen und dann nach jedem die Technik korrigiert." (173)

Die Hauptkategorie „Ärzte, die die Ausbildung der Studierenden ernst nehmen“ umfasst Aussagen, die die Vorbildlichkeit von lehrenden Ärzten betonten, die der Lehre einen hohen Stellenwert beimaßen und qualitativ hochwertige Lehrveranstaltungen anboten, und neben theoretischen besonders auch soziale Lerninhalte vermittelten. In der Hauptkategorie zeigen die Aussagen „Ärzte, die in Lehrsituationen Studierende für den Beruf begeistern", dass als vorbildlich gewertete Lehrveranstaltungen den Studierenden nicht nur Freude bereiteten, sondern auch eine motivierende Wirkung hatten und ihr Interesse für den Beruf weckten. Vorbildlich war für die Studierenden auch eine große Berufszufriedenheit von Ärzten. So antworteten mehrere Studierende, es habe eine Vorbildfunktion für sie, wenn Ärzte „Liebe zum Beruf“ zeigten.

In der Hauptkategorie „Ärzte, die Führungskompetenz und Kollegialität vereinen“ geht es um den Führungsstil ranghoher Ärzte, die ein gutes Verhältnis zu Mitarbeitern wahren. Einen respektvollen Umgang mit rangniedrigeren Mitarbeitern und Interesse an ihrer Ausbildung war für die Studierenden ein Vorbild.

Die Hauptkategorie „Ethische Grundprinzipien medizinischen Handelns implizit vermitteln" beinhaltet das Vorleben humanitärer Einstellungen wie "Gleichberechtigung aller Menschen“, ein Patientenumgang mit "Würde und Menschlichkeit" und der Einsatz für "das Wohl der Patienten“. Studierende erachteten es ebenfalls als vorbildlich, wenn Ärzte „Unsicherheit, Fehler und die Begrenztheit des eigenen Wissens eingestehen“.

Eine Besonderheit ist die Hauptkategorie „Ärzte, die in der medizinischen Hierarchie oben stehen", da Ärzte (wie oben erwähnt) teilweise aufgrund ihrer Position als Vorbilder gewertet wurden. Im Speziellen fanden einige namentlich genannte Professoren Erwähnung, ohne dass jedoch erklärt wurde, weshalb sie vorbildlich waren. Hier wurde auch die Kategorie „Vorbildfunktion von Ärzten aufgrund ihres Wissensvorsprungs gegenüber Studierenden" verortet, da auch diese Bezug auf Unterschiede der Positionen innerhalb einer Hierarchie nimmt. 
Ergebnisse

Die von den Studierenden hervorgehobene Eigenschaft ranghoher Ärzte, trotz ihrer Position Interesse an Studierenden und besondere persönliche Eigenschaften zu bewahren, führte zur Bildung einer zusätzlichen Hauptkategorie: „Ärzte, die trotz ihres hohen Ranges Interesse an Studierenden haben und durch ihre Persönlichkeit Vorbilder sind“; hierunter fiel auch ihr „persönliches Engagement“ für die Lehre und "Studentennähe".

Unter der Hauptkategorie „Kommilitonen, die engagiert, kompetent und unterstützend sind" erscheint eine neue Personengruppe als Vorbild. Kommilitonen waren sowohl aufgrund von Charaktereigenschaften wie „Fleiß und Hilfsbereitschaft", als auch wegen „fachlicher" Kompetenzen und effektiver Lernstrategien Vorbild. Die Vorbildfunktion studentischer Hilfskräfte bezog sich auf inr Wissen und ihre Bereitschaft, Wissen und Erfahrung bereitwillig mit Jüngeren zu teilen. Vorbilder wurden Kommilitonen auch wegen sozialer Kompetenzen und besonderer Einstellungen zur Medizin, z.B. nachahmenswerter Motivation fürs Studium.

\subsection{Ergebnisse der Interviewauswertung}

Die Ergebnisse der ELSAS-Studie waren eine Voraussetzung bzw. Anregung für Themen, die im Rahmen von Interviews detaillierter exploriert wurden. Deren Analyse führte zu der Identifizierung zentraler Phänomene, auf die in den folgenden Kapiteln einzeln eingegangen wird. Für den Aufbau dieser Kapitel wurde eine einheitliche Struktur gewählt, orientiert an der Grounded Theory am Kodier-Paradigma (Strauss und Corbin 1996), (s. Kap. 4.2.6: „Durchführung der Auswertung“). Zu Beginn jedes Kapitels steht eine kurze Beschreibung des Phänomens. Im Anschluss folgen Bestandteile des Kodier-Paradigmas (Kontext, ursächliche Bedingungen, intervenierende Bedingungen, Strategien und Konsequenzen).

\subsubsection{Orientierungshilfen durch Vorbilder: Die Rolle der Kommilitonen}

\section{Das Phänomen}

Auf die Frage nach Erfahrungen mit Menschen, die für Medizinstudierende und ihre Ausbildung von Bedeutung waren, wurden zumeist erste Kontakte zu Kommilitonen während der Orientierungsphase genannt. Die Kontinuität dieser Kontakte während des Studiums war den Studierenden wichtig und blieb im Gegensatz zu Kontakten zu Dozenten auch häufig erhalten. 
Ergebnisse

Besonders vermittelten ältere Studenten Umgangsformen und bestimmte Denk-, Handlungs- und Wertmuster, die im Medizinstudium vorherrschen. Auch in Bezug auf andere Bereiche des Studiums (z.B. Unipolitik und Forschung S08 09) waren sie hilfreich. Dies blieb nicht auf fachliche Inhalte des Studiums beschränkt, sondern bezog auch z.B. die Kontaktaufnahme mit anderen Studenten oder Einstellungen zum Studium mit ein:

„Dann kam eben X-(Name der Freundin 5) die das alles ein bisschen leichter gesehen hat als ich [...] und sie war so ein bisschen unbeschwerter einfach //l:mhm// was das alles anging also sie war auch mit der Sprache nicht so sie hatte das noch nicht so drauf wie ich und es war ihr völlig egal und ähm .. ja und das hat mich glaub ich zunächst so fasziniert" (S11 055) ${ }^{19}$.

\section{Kontext}

Viele Studierende berichteten, zu Beginn des Studiums in Anbetracht mangelnder Erfahrungen mit Anforderungen unsicher und überfordert gewesen zu sein (S15 007). Student S07 erlebte das Studium als anonym und sah den Austausch mit Kommilitonen als Möglichkeit, "das Soziale“ ins Studium zu bringen (S07 10). S10 beschrieb, wie er über Gespräche mit Vertrauenspersonen im Studium „Stress abbauen" konnte (S10 052). Auch andere Studenten berichteten von ihrem Bedürfnis nach Verständnis für die eigene Situation im Studium. Dies wurde vor allem von Kommilitonen erfült, die das Studium aus einer gemeinsamen Erfahrungswelt heraus erlebten (S07 10) und daher den Mitstudierenden besonders zu Beginn eine erste Orientierung und Sicherheit im Studium geben konnten (S16 003).

\section{Ursächliche Bedingungen}

Die Studierenden hatten ein Bedürfnis nach Unterstützung im Studium. Oft bestand Zweifel bezüglich des eigenen Könnens und der Bewältigung des Studiums, und sie litten unter Leistungsdruck (vgl. S15 027). Sie berichteten von mangelnden Kontakten zu Dozenten und dem Gefühl, von ihnen nicht wahrgenommen zu werden. Ihre Anwesenheit in Lehrveranstaltungen schien gleichgültig zu sein. Dozenten wurden häufig als Prüfer erlebt, Kommilitonen hingegen stünden auf derselben Seite.

Die Angst, im Studium zu versagen, wurde durch einige der Dozenten eher verstärkt, als dass sie innen bei der Bewältigung halfen (S12 04).

\footnotetext{
${ }^{19}$ Nummer des Zitates (vgl. Fußnote 14).
} 
Ergebnisse

„vielleicht müssen sie es so machen um zu selektieren aber ähm ich denke gerade am Anfang vom Studium ist man noch relativ jung und vielleicht noch nicht so abgehärtet und //I:mhm// ähm... ja also ich finde da musste man schon lernen“ (S05 091).

Die Bereitschaft der Studierenden, sich an Kommilitonen zu orientieren, schien ein Vertrauen in diese Person vorauszusetzen. Dies beinhaltete zum einen, offen mit Wissenslücken oder mit eigenen Fehlern umgehen zu können (S13 076). S15 berichtete, eine gute Vertrauensbasis mit ihren Hiwis zu haben, die es ihr erlaubte, ihre Hilfe in Situationen am Präparier-Tisch anzunehmen, in denen es ihr schlecht ging (vgl. S15 031). Eine Vertrauensbasis mit Kommilitonen beinhaltete für die Studierenden auch, sich verstanden und unterstützt zu fühlen:

„ich wüsste nicht genau wie das wäre wenn ich jetzt äh diese Leute nicht hätte //l:Ja// also dann glaub ich würd ich mich sehr unwohl fühlen ähm genau also geben mir ne soziale Sicherheit //l:Ja // ähm und dadurch dass sie das Gleiche tun wie ich verstehen sie eventuell auch in der Situation ähm gut was ich eben empfinde (S07 10)."

\section{Intervenierende Bedingungen}

Ältere Studierende wurden in verschiedenen Funktionen erlebt, z.B. als Tutoren und Hilfswissenschaftler in ihrer Funktion als studentische Lehrkräfte. Häufig wurden Hiwis wie auch Kommilitonen als „Verbündete“ erlebt und war ihr Rat und Antrieb zum Lernen aufgrund der bestehenden Vertrauensbasis leichter annehmbar als von Dozenten (S07 42).

Die Studienteilnehmer benannten ebenso negative Erfahrungen mit Kommilitonen: beispielsweise Konkurrenzverhalten (S06 11) und berichteten auch von Unterschieden bezüglich der eigenen Stellung in der medizinischen Hierarchie innerhalb der Studentenschaft:

„....und hab mir sehr viel Stress gemacht und das war auch nicht gut und dann waren viele $\mathrm{Hi}$ wis dabei die den Stress einfach nur noch größer gemacht haben [...] ich find das unmöglich ich weiß nicht warum die so sind die waren ja auch nur ein zwei Semester weiter s.o.[...] also ich finde Hierarchien gibt's ja genug //l:ja// die muss man ja nicht noch künstlich aufbauen indem man sich aufspielt nur weil man zwei Semester weiter ist (S15 072).“

Lernpartner wurden auch nach Wissensstand oder Zuverlässigkeit ausgesucht. S16 beschreibt seine Lernpartner beispielsweise als „pflichtbewusst“ und "gewissenhaft“:

„genau ja also äm wir haben halt gemerkt dass es also wir sind so äh drei vier Mann wir sind so relativ der gleiche Lerntyp und wir haben halt gemerkt dass es also für uns wir sind so drei 
Ergebnisse

vier Jungs und für uns ist es halt sinnvoller zusammen zu lernen äm weil wir meistens unser Wissen ergänzen können //l: $\mathrm{mhm} / /$ und das ist dann äm halt effektiver und schneller" (S16 $15)$.

\section{Handlungsstrategien}

Häufig verglichen die Studierenden sich untereinander und orientierten sich am beobachteten Verhalten anderer Studierender. Vorbildhafte Kommilitonen verfügten häufig über bestimmte Fähigkeiten oder Eigenschaften, die besonders geschätzt wurden (wie z.B. ihre Kompetenz S08 84, ihr Engagement für das Studium und für andere, ihre Erfahrenheit S07 55 oder ihr Organisationstalent S14 143). Von innen und von älteren, erfolgreichen Kommilitonen schauten sich die Studierenden Strategien zur Bewältigung des Studiums ab. Dazu gehörte es, Ratschläge in Anspruch zu nehmen (beispielsweise was die Vorbereitung auf eine Klausur angeht $S 10$ 091) und Lernstrategien zu übernehmen. Dazu gehörte auch die gemeinsame Bewältigung von Anforderungen. S13 vertraute der Kompetenz seiner Lernpartner und der Effizienz seiner Lerngruppe. Das gemeinsame Lernen in der Lerngruppe wurde von inm als effektiver wahrgenommen als offizielle Lehrveranstaltungen wie Vorlesungen.

Zu den Strategien der Studierenden gehörte es auch, sich Hilfe bei der Bewältigung von Problemen im Studium zu holen (S05 095). Neben Lernstrategien gehörte dazu auch der Umgang mit Zeitnot und Stress. Einige erlebten es als hilfreich, zusammenzuhalten und das Studium gemeinsam zu bewältigen (S09 07). Andere gaben an, auch Konkurrenzverhalten von Kommilitonen erfolgreich gelernt zu haben. S04 bewunderte seinen Freund, den er als „Anpacker“ bezeichnete, für seinen Ehrgeiz und seine Einstellung, sich Herausforderungen zu stellen und sich auch mal "durchzuboxen" (S04 003). Auch andere Studierende empfanden es als wichtig, sich gegenseitig anzuspornen und zum Lernen zu motivieren (S13 080).

\footnotetext{
„also wir haben.. zusammen .. als wir lernen mussten einfach recht viel Zeit in der Bibliothek verbracht zusammen und haben dann so uns so gegenseitig motiviert //l:mhm// und.. wir haben ein bisschen die Metapher gefunden für die Anatomie als Drachen den wir so bezwingen können“ (S04 031).
}

Die Orientierung z.B. an Kommilitonen in Bezug auf die eigene „Rolle“ als Studentin/Student, wird im Kapitel 5.2.5: Rolleneinübung: Auseinandersetzung mit der Rolle als Medizinstudent/in und Antizipation der Arztrolle beschrieben. 


\section{Konsequenzen}

Die Studienteilnehmer waren sich vor allem der Wichtigkeit von Kommilitonen in Bezug auf Unterstützung und sozialen Rückhalt bewusst. So berichteten sie, dass persönliche Kontakte zu Freunden und Kommilitonen zu einem Gefühl von Sicherheit im Hinblick auf die eigene Situation im Studium führten. Sie vermittelten innen den Eindruck, Anforderungen nicht allein bewältigen zu müssen (S07 10) und motivierten sie, zu lernen und durchzuhalten:

„....uns dann sozusagen zu viert dann eigentlich durch die Vorklinik geschlagen haben und.. ich dann vor allem mit einer Freundin also X-(Name der Freundin) heißt sie ähm... ja.. also ja.. sie für mich einfach ne ganz wichtige Person geworden ist und ähm ich denke wenn man.. das wenn ich das irgendwie alleine gemacht hätte so.. dann wär's einfach... ähm viel anstrengender gewesen und es hätte... einen einfach viel mehr mitgenommen auch mehr Unsicherheit geschaffen also wenn man da zusammen durchgeht dann ist das einfach was anderes (S03 03).“

Die Bewältigung des Studiums wurde in der Wahrnehmung der Studierenden durch ihre Kommilitonen erleichtert. Neben der Vermittlung von Zuversicht, das Studium schaffen zu können, konnten Kommilitonen auch dabei halfen, Selbstzweifel und Unsicherheiten abzubauen (S01 41). Neben persönlicher Hilfestellung und Unterstützung vermittelten Kommilitonen auch den „richtigen“ Umgang mit dem Studium.

Die Studierenden erhielten durch ältere Studenten ein realistisches Bild von Anforderungen im Studium (S16 003) und lernten von Kommilitonen einen besseren Umgang mit sich selbst im Kontext des Studiums. S03 berichtete, von ihrem Freund gelernt zu haben, „auf sich selber zu achten“ (vgl. S03 43). S03 erzählte, dass sie sich mit ihrer Lernpartnerin gegenseitig darin bestärkte, einen Ausgleich zum Studium zu suchen, den sie sich ohne sie aus Zeitmangel nicht erlaubt hätte (S03 27). Eine andere Studentin empfand es als besonders wichtig zu lernen, sich nicht durch das ,GestresstSein' anderer Kommilitonen anstecken zu lassen. Ihre Freundin habe sie mit ihrer "Coolness" angesteckt (S11 031-39).

Aber auch andersherum konnte es sein: Hiwis, die den „Stress (für die Studenten) einfach nur noch größer gemacht haben“ (S15 027), oder Medizinstudierende, die als „super straight" beschrieben wurden und durch ihre besonders ehrgeizige Einstellung zum Studium vorbildhaft waren.

„[...] es gibt nur noch das und ich muss einfach alles verstehen" (S11 027). 
Ergebnisse

Die Vorbildwirkung von Kommilitonen zielte folglich nicht allein auf Stressabbau und bewältigung, sondern konnte durch bewunderten Ehrgeiz, Selbstzweifel und das Gefühl, unter Druck zu stehen, auch steigern.

Der Austausch mit Kommilitonen hatte für die Studierenden nicht nur im Hinblick auf das Studium, sondern auch auf ihre berufliche Entwicklung Bedeutung (S13 080). Im Austausch mit Kommilitonen lernten sie ihre Einstellungen, Schwerpunkte und Ziele kennen, die ihre eigene Wahrnehmung erweiterten:

„....dass wir uns da eigentlich ham wir uns immer gut austauschen können über Dinge die wir erlebt haben //I:mhm// hat er auch manchmal ähnlich gesehen und das hab ich auch viel von inm lernen können dass er .. wie soll ich sagen vielleicht noch viel mehr als ich hat er versteht er Medizin glaub ich sehr zwischenmenschlich sehr auf den Menschen bezogen während ich vielleicht die Sachen eher biologischer sehe //l: ja// konnt ich da viel von ihm lernen glaub ich wie man halt irgendwie auf die Menschen sehr achten muss bei den Dingen (S08 28)."

Ältere Studierende schienen einen Einfluss darauf zu haben, dass Student S13 sich von Beginn an dem Kreis der Medizinstudenten zugehörig fühlte. Auch S16 berichtete davon, von ihnen viel Hilfe und eine erste Orientierung erhalten zu haben:

„von daher ist diese O-Phase ausschlaggebend für das was da zur Zeit so los ist bei mir“ (S16 03).

Zudem schien er sich auch mit seinen Kommilitonen und Lernpartnern zu identifizieren (vgl. S16 15). S12 hingegen identifizierte sich weder mit dem Studium noch mit ihren Kommilitonen (vgl. auch Kapitel 5.2.3: Identifikation mit Ärzten, Studium und Beruf)

,ja und ich bin halt auch irgendwie in diesem Medizinstudium gelandet ((lacht)) //l:mhm// und steh da aber auch nicht ganz hinter" (S12 46).

Sie fühlte sich „anders“ als ihre Kommilitonen und meinte, dass solche „Zweckkontakte" nicht für eine Freundschaft ausreichen (S12 13, 15 und 04). Andere Studierende, die nicht zwischen Freundschaft und Lernbeziehung trennten, berichteten, dass Freunde ganz automatisch auch zu Lernpartnern wurden (vgl. u.a. S07 10, S09 15). S12 schien sich nicht an ihren Kommilitonen orientieren zu wollen. Abbildung 3 fasst die Ergebnisse zur 1. Hauptkategorie im Sinne des Kodier-Paradigmas zusammen: 


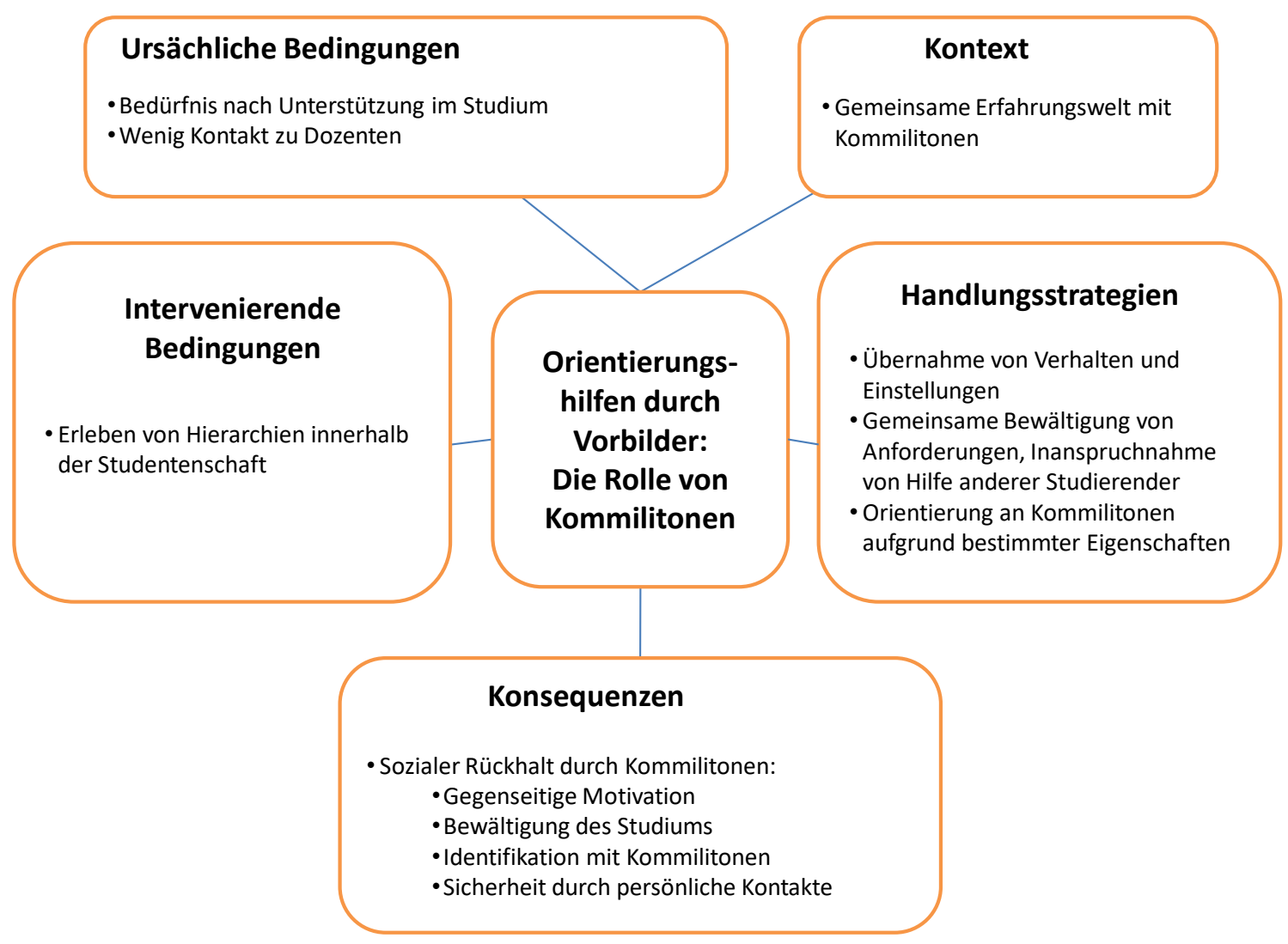

Abbildung 3: Kodier-Paradigma zum Thema „Orientierungshilfen durch Vorbilder: Die Rolle von Kommilitonen“.

\subsubsection{Orientierungshilfen durch Vorbilder: Die Rolle von Lehrenden im Studi- um und für den Beruf}

\section{Das Phänomen}

Mehrere Studierende berichteten von Orientierungslosigkeit und Unsicherheit zu Beginn des Studiums. Sie schilderten mit Blick auf die Anforderungen im Studium, dass sie noch nicht wussten, „worauf sie sich eingelassen“ haben (S15 007) und dass sie lernen mussten, „sich durchzukämpfen“ (S05 091). Einige berichteten von Unsicherheit und Orientierungslosigkeit aufgrund mangelnder Erfahrungen im Krankenhaus und einer fehlenden Betreuung bei ersten Kontakten mit der klinischen Arbeit im Rahmen von Praktika (vgl. S06 55 und S15 055).

In dieser neuen Situation hatten häufig Kommilitonen eine unterstützende Rolle (vgl. voriges Kapitel: Orientierungshilfen durch Vorbilder: Die Rolle von Kommilitonen). Dozenten hatten in dieser Phase weniger direkte Kontakte zu den Studierenden. 
Ergebnisse

Trotzdem spielten auch sie eine Rolle als Orientierungshilfe. Häufig waren sie diejenigen, die den Studierenden während ihrer ersten klinischen Erfahrungen reale Eindrücke des Berufs vermittelten. Sie dienten den Studierenden als Beispiele für ärztliches Verhalten in der neuen Umgebung des Krankenhauses. Wenn sie gelungene Interaktionen zwischen Patient und Arzt beobachteten, war dies für Studierende eine "einschneidende" und motivierende Erfahrung (vgl. u.a. S06 03). Darüber hinaus fungierten Ärzte und Dozenten als Orientierungshilfe für die eigene berufliche Zukunft der Studierenden, besonders wenn ein klares und zutreffendes Bild des angestrebten Berufes fehlte.

\section{Kontext}

Mehrere Studierende artikulierten ein deutliches Bedürfnis nach Orientierungshilfe; dies richtete sich besonders an Ärzte im Krankenhaus und Dozenten ${ }^{20}$ in Lehrveranstaltungen z.B. in Form von direkten Gesprächen im Anschluss an ein Seminar. Zu solchen persönlichen Gesprächen zwischen Student und Vorbild kam es selten, häufig aber wurden Ärzte während ihrer Arbeitsroutine beobachtet. Ein Bedürfnis nach Orientierungshilfen entstand oftmals in überfordernden und als belastend erlebten Situationen, insbesondere zu Beginn des Studiums und während erster Erfahrungen im Krankenhaus, aber auch in Lern- und Prüfungssituationen (vgl. 5.2.1: Orientierungshilfen durch Vorbilder: Die Rolle von Kommilitonen und 5.2.4: Auseinandersetzung mit Idealen der ärztlichen Profession).

\section{Ursächliche Bedingungen}

Distanz zu ihren Dozenten veranlasste die Studierenden dazu, Unterstützung bei der Bewältigung des Studiums vor allem bei ihren Kommilitonen zu suchen (vgl. Kapitel 5.2.1: Orientierungshilfen durch Vorbilder: Die Rolle von Kommilitonen), da sie „als auf der gleichen Seite stehend" wahrgenommen wurden. Die Studierenden äußerten jedoch auch den Wunsch nach einem persönlicheren Kontakt zu ihren Dozenten. Sie interessierten sich für die Persönlichkeit von Ärzten (vgl. Kapitel 5.2.3: Identifikation mit Ärzten, Studium und Beruf) und ihr Verhalten im Privaten. So war dann für S14 eine befreundete Radiologin, die sie als "nette Frau“ beschrieb, deren „stilvolles“ Leben jedoch nicht zu ihrem Lebensentwurf passte, kein Vorbild (vgl. S14 099). Ein

\footnotetext{
${ }^{20}$ Als „Dozenten“ werden hier sowohl Lehrkräfte der Fakultät in regulären Lehrveranstaltungen, als auch Lehrärzte in Kliniken bezeichnet, denen Studierende während Praktika begegneten.
} 
Ergebnisse

anderes Beispiel dafür, dass eine Orientierung an Lehrenden nicht nur in Bezug auf ihre professionelle Rolle erfolgte, ist das Interesse von S04 am Verhalten eines Professors seiner Frau gegenüber (vgl. S04 091).

Die Beobachtung von ärztlichen Vorbildern war auch eine Möglichkeit, ärztliches Verhalten unter realen Bedingungen kennen zu lernen (vgl. Kapitel 5.2.5: Rolleneinübung: Auseinandersetzung mit der Rolle als Medizinstudent/in und Antizipation der Arztrolle). Student S08 berichtete von Erfahrungen aus seinem Praktischen Jahr:

„in der Pathologie .. das war ne gute Zeit //I:mhm// .. da ham mich viel der X-(Name eines Arztes 2) heißt der und der hat sehr viel mit uns gemacht hat sich viel mit uns beschäftigt hat uns viel beigebracht //I:mhm// hat uns auch so das Wesen vom Pathologen so wie der einfach arbeitet uns viel gezeigt“ (S08 16).

Nicht so sehr Faktenwissen war für S08 in der Suche nach Vorbildern entscheidend, sondern das Charakteristische am Arztberuf kennen zu lernen. Sich ein konkretes Bild des Berufes zu machen, schien bereits Teil der Vorbereitung auf den Beruf zu sein. Unsicherheit und Zweifel bezüglich des eigenen Vermögens, die Rolle als Arzt auszufüllen, nahmen unmittelbar vor dem Ende des Studiums zu:

„Naja was einen zum Beispiel immer so ein bisschen ärgert dass man am Anfang vom Studium denkt man hätte ne Ahnung von allem so grob um umso näher das Ende des Studiums rückt umso mehr hat man das Gefühl man weiß irgendwie fast gar nichts“ (S01 42).

Auch S04 fühlte sich mit seinem derzeitigen Kenntnisstand dem späteren Beruf nicht gewachsen. Er bewunderte Kommilitonen, die bereits als Rettungssanitäter in der Notfallmedizin tätig waren; zugleich beschrieb er den zukünftigen Beruf als „bedrohlich“ und sagte, dass es ihm „ganz schön Respekt eingeflößt“ habe, „völlig allein in der Verantwortung zu stehen“ (S04 007).

\section{Intervenierende Bedingungen}

Dozenten als Orientierungshilfe zu finden, beschrieben die Studierenden als schwierig (vgl. Kapitel 5.2.4: Auseinandersetzung mit Idealen der ärztlichen Profession). Gründe hierfür waren die Distanz zu Lehrenden und die fehlende Beachtung durch sie. Von Dozenten in Lehrveranstaltungen wahrgenommen, verstanden und unterstützt zu werden, empfanden mehrere Studierende als Ausnahme (S05 095, S13 053).

Im Vergleich zur Beziehung der Studierenden zu Kommilitonen (s. Kapitel 5.2.1: Orientierungshilfen durch Vorbilder: Die Rolle von Kommilitonen) beschrieben die 
Ergebnisse

Studierenden das Verhältnis zu Mitgliedern der Fakultät, vor allem mit ",hohem Rang“21 häufig als distanziert. Sie fühlten sich Dozenten gegenüber häufig unterlegen. Dies bezog sich vor allem auf wahrgenommene Wissens- und Erfahrungsunterschiede. Viele Studierende erzählten, wenig persönlichen Kontakt mit Professoren zu haben und sich von innen „eingeschüchtert" zu fühlen (S12 04); besonders unter Prüfungssituationen erlebten sie Dozenten als „Prüfer“, die Studenten "selektierten“ (S05 091). Professoren würden sich innen gegenüber auch in Lehrveranstaltungen distanziert verhalten. S04 hatte den Eindruck, dass ein Professor in seinem Wahlfach seine Geschichte nicht „übermäßig persönlich“ erzählen wollte (S04 09) und lediglich persönliche Dinge preisgab, die einen fachlichen Zusammenhang mit den von ihm unterrichteten Lerninhalten hatten. Hinzu kam, dass Dozenten häufig als Negativbeispiele für ärztliches Verhalten erlebt wurden (s. Kapitel 5.2.4: Auseinandersetzung mit Idealen der ärztlichen Profession).

\section{Handlungsstrategien}

Orientierung im Studium und für die weitere berufliche Entwicklung zu finden, wurde als wichtiges Ziel angesehen. Im Zusammenhang mit Überlegungen, welche fachliche Spezialisierung für inn in Frage käme, äußerte S13:

„und so grabe ich mir da meinen Weg und hoffe dass ich dann irgendwie fertig bin //l:mhm// und dann weiß was ich machen will" (S13 003). ${ }^{22}$

Ob das Verhalten der beobachteten Ärzte nachahmenswert erschien, war entscheidend für die Eignung als Vorbild. Vorstellungen der Studierenden über „ärztlich korrektes Verhalten" und ihre Erwartungen an Lehrkräfte hatten Einfluss auf deren Bewertung als "Orientierungshilfe". In den Beschreibungen der Studierenden zeigten sich klare Vorstellungen darüber, welches Verhalten von Ärzten richtig oder falsch ist. Eine Studentin sagte beispielsweise über eine beobachtete Assistenzärztin, dass diese „falsch mit der Situation umgegangen“ sei (S11 083). Die Studierende schienen sich gezielt Personen auszuwählen, die sie inspirierten. S08 hatte ein Vorbild gefunden, dem es lohnt nachzueifern:

„Also der X-(Name eines Kommilitonen) ist sicherlich ein Vorbild für mich //I:mhm// der ist vielleicht sogar ja .. wie soll ich sagen der ist vielleicht so an der Stelle wo der so oder so ... weiß

\footnotetext{
21 „Rang“ meint die Position der Ärzte innerhalb der Hierarchie eines Krankenhauses (z.B. Oberarzt).

${ }^{22}$ Erklärung der Transkriptionsregeln siehe Anhang 8.5.
} 
Ergebnisse

ich nicht so gut einfach dass ich denke da will ich vielleicht gar nicht ganz drankommen //I:mhm// das was er tut //I: okay// aber deswegen vielleicht grade deswegen ist es auch Vorbild weil ich denke da kann ich zumindest ähm mich ran arbeiten //l:mhm// grad was Forschung angeht sozusagen oder wie auch sozusagen man so $n$ Weg gehen kann //I:mhm// innerhalb von Klinik und Forschung wann man welche Schritte machen muss .. das ist ganz sicher ein Vorbild für mich //l: ja// .. und also vielleicht ist ja doch ist das ein wichtiges Vorbild //l:mhm// weil das .. auch vielleicht noch so nah ist sozusagen schon dass ich schon noch verstehe was passiert //I:mhm// dass ich das nachempfinden kann vielleicht nicht jeden nicht alles schaffe nachzumachen sozusagen //l: ja// aber .. schon mir da viel ähm dran lernen konnte“ (S08 84).

Studentin S05 hatte ebenfalls klare Vorstellungen davon, wer für sie ein „guter" und wer ein „schlechter Arzt“ ist. Sie suchte den Kontakt zu einer Ärztin, die es schafft, die Studierenden mit der Begeisterung für ihr Fach anzustecken (vgl. S05 111). Auch der Entschluss, sich nicht an einem Arzt zu orientieren, den sie als Negativbeispiel identifizierte, wirkte bei ihr wie eine Handlungsstrategie und war Bestandteil einer aktiven Orientierungssuche. Das folgende Zitat ist eine Reaktion der Studentin auf eine Situation während ihres Pflegepraktikums, in der der Arzt das Zimmer, in dem sich eine Patientin gerade übergibt, sofort wieder verlässt, ohne sich um sie zu kümmern:

„Ja also ähm in meinen Augen egal was man macht denke ich ähm wenn's nen ob Patient oder jemand auf der Straße ist dem es schlecht geht //l:mhm// dann versucht man den zu unterstützen egal ob man im medizinischen Bereich ist oder nicht und äh wenn man schon im medizinischen Bereich tätig ist wie dieser Arzt von dem ich das Beispiel genannt hab //l:mhm// und der genau das Gegenteil macht von dem was man eigentlich als Mensch machen würde..//l:mhm// dann ist der in meinen Augen fehl am Platz und [...] dass er sich als was Besseres fühlt auf jeden Fall war das für mich so verletzend wie er mit diesem Kind was wirklich krank war //l:mhm// umgegangen ist [...] ja wirklich unmenschlich und auch so herablassend“ (S05 31).

Die emotionale Reaktion der Studentin („fehl am Platz“) spricht für den Wunsch, es selbst als angehende Ärztin anders machen zu wollen.

\section{Konsequenzen}

Kontakte zu Ärzten, die als Orientierungshilfe oder sogar Vorbilder wahrgenommen wurden, führten bei den Studierenden häufig zu Zufriedenheit und Motivation im Studium (vgl. u.a. S11 099, S14 111, S03 73). S13 beschrieb einen Arzt, der sich bemühte, ihn bei seiner Arbeit mit einzubeziehen, als „Auslöser“ dafür, Interesse am 
Patientenkontakt entwickelt und seine Motivation für das Studium grundlegend verändert zu haben. S05 berichtete von einer Professorin, die den Studierenden das Gefühl vermittelte, von Bedeutung zu sein und sie persönlich unterstützte. S05 sagte, sie habe ihr dabei geholfen, nicht „aufzugeben“ (S05 011) und Mut gemacht, indem sie ihr „Tipps“ gab, „wie sie selbst mit der Situation umgegangen ist“. Sie bewies ihr durch ihr Beispiel, dass es möglich ist, Schwierigkeiten im Studium wie Prüfungsangst zu überwinden. Dass wie in diesen Beispielen eine Person gefunden wurde, die alle von den Studierenden angestrebten Ziele und Erwartungen in sich vereinte, war jedoch selten.

Freiwilligkeit und Vertrauen schienen bei dem Entschluss, sich an bestimmten Verhaltensgrundsätzen von Dozenten zu orientieren, von Bedeutung zu sein. Ein Arzt, der auf die Unfähigkeit der Studentin, seine Frage zu beantworten, mit den Worten, „das müssen Sie [...] wissen“ reagierte, schien S14 nicht dazu zu motivieren, mehr Engagement für das Studium zu zeigen. Auch sein Verhalten am Operationstisch wertete die Studentin keineswegs als vorbildlich (vgl. S14 114-124). Die Ratschläge einer als engagiert wahrgenommenen studentischen Hilfskraft, über die der Student S07 sagte, sie habe inn zum Lernen „motiviert“, konnte er hingegen aufgrund einer bestehenden Vertrauensbasis und des Umgangs miteinander besser annehmen (S07 43). Auch ein Verhalten von Professoren, bei denen nicht Macht demonstriert und auf Maßnahmen verzichtet wurde, die die Studierenden unter Druck setzen, wurden geschätzt. Gerade nicht erzwungene Autorität zeichnete sie als Vorbilder aus.

Das Fehlen von Ärzten als Orientierungshilfe im Studium und besonders im Rahmen erster Patientenkontakte schien die oben beschriebene Unsicherheit der Studierenden noch zu verstärken. So fühlten sie sich in den entsprechenden klinischen Situationen überfordert und konnten sich nicht ihren eigenen Erwartungen gemäß „professionell“ verhalten (vgl. Kapitel 5.2.5: Rolleneinübung: Auseinandersetzung mit der Rolle als Medizinstudent/in und Antizipation der Arztrolle).

Sich am Verhalten und Erleben des Krankenhauspersonals im Arbeitsalltag zu orientieren, verhalf den Studierenden zu einem konkreteren Bild des angestrebten Berufes. Sowohl positive als auch negative Erfahrungen im Studium schienen die Ausrichtung der Studierenden zu beeinflussen und zu einer Reflexion eigener Vorstellungen und einer Veränderung von Erwartungen an den angestrebten Beruf zu füh- 
Ergebnisse

ren. Ärzte, an denen sich die Studierenden orientierten, wurden nicht unbedingt auch als Vorbilder angesehen. Prägend waren Erfahrungen mit innen jedoch trotzdem (vgl. Kapitel 5.2.5: Rolleneinübung: Auseinandersetzung mit der Rolle als Medizinstudent/in und Antizipation der Arztrolle). Wie im Fall von Studentin S14 vermitteln sie ein neues, möglicherweise realistischeres Bild des Arztberufes, das jedoch ambivalenter ist als die bisherigen Idealvorstellungen. S14 äußerte keine eindeutige Einstellung zu dem Verhalten eines Arztes, den sie zunächst als „total nett“ beschrieb, dessen Verhalten (während der Operation) und Umgang mit Studierenden sie jedoch als „fragwürdig“ bewertete, da er vorwurfsvoll darauf reagierte, dass sie seine Fragen nicht beantworten konnte (vgl. S14 111 und 119).

Abbildung 4 fasst die Ergebnisse zur 2. Hauptkategorie im Sinne des KodierParadigmas zusammen:

\section{Ursächliche Bedingungen}

- Unsicherheit zu Beginn des Studiums

- Kennenlernen ärztlichen Verhaltens unter realen Bedingungen

- Notwendigkeit der Vorbereitung auf den bevorstehenden Beruf

\section{Kontext}

- Bedürfnis nach Orientierungshilfen besonders in belastenden Situationen

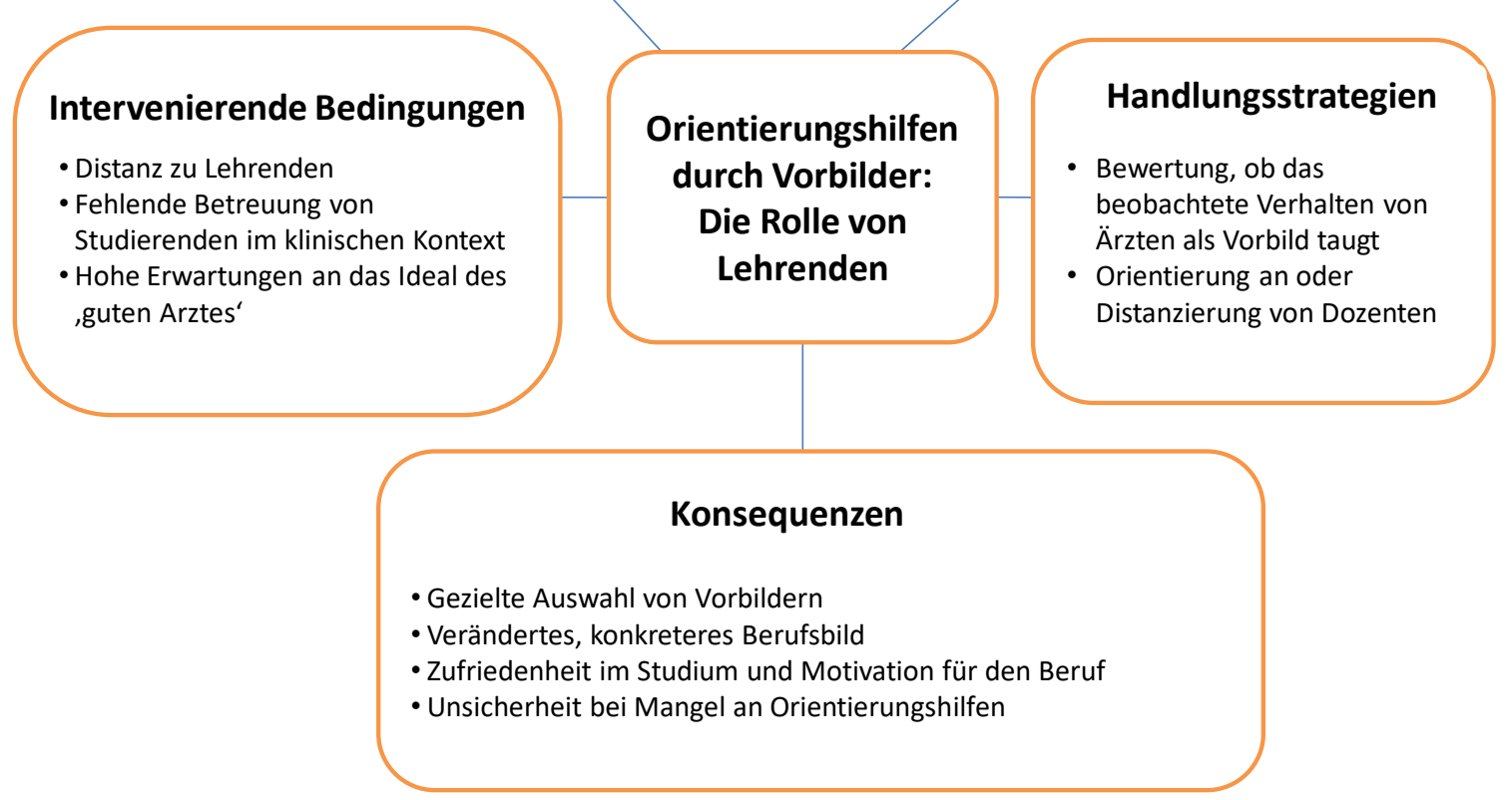

Abbildung 4: Kodier-Paradigma zum Thema „Orientierungshilfen durch Vorbilder: Die Rolle von Lehrenden im Studium und für den Beruf“. 


\subsubsection{Identifikation mit Ärzten, Studium und Beruf}

\section{Das Phänomen}

,Identifikation“ war ein wichtiges Thema für viele Studierende: sowohl mit prägenden Personen als auch um die Identifikation mit dem Studium und dem Arztberuf. Identifikationen wurden häufig im Gesamtzusammenhang ersichtlich, jedoch von den Studierenden selten klar benannt. Identifikation mit dem Studium z.B. beschrieben Studierende als Gefühl, am richtigen Ort zu sein, oder drückten sie in lobenden Worten oder Zufriedenheit mit dem Studium aus. Der Grad der Identifikation mit dem Studium hing damit zusammen, ob und wie stark sich die Studierenden mit anderen Mitgliedern der Fakultät identifizierten. Dasselbe galt für die Identifikation mit der Arztrolle (s. bes. Kapitel 5.2.5: Rolleneinübung: Auseinandersetzung mit der Rolle als Medizinstudent/in und Antizipation der Arztrolle). Bestimmte Eigenschaften und Qualitäten von Ärzten erleichterten die Identifikation und bezogen sich häufig auf das Verhalten der Ärzte den Studierenden und den Patienten gegenüber. Die Studierenden charakterisierten solches Verhalten Patienten gegenüber mit Begriffen wie "ganzheitlich“, „empathisch“, „auf einer Augenhöhe“ oder „authentisch“. Identifikation mit Dozenten und damit Vorbildfunktion setzte voraus, dass Ärzte Interesse an Studierende zeigten (vgl. S13 108 u. 52, S11 075), z.B. durch Engagement für die Lehre und Unterstützung bei der Bewältigung von Schwierigkeiten im Studium (vgl. S05 095).

Eine Identifikation mit Ärzten war Konsequenz eines Prozesses der Auseinandersetzung mit ihnen, gleichzeitig jedoch auch Voraussetzung weiterer Identifikationsprozesse, z.B. mit dem Studium und der Arztrolle (s. Kapitel 5.2.5: Rolleneinübung: Auseinandersetzung mit der Rolle als Medizinstudent/in und Antizipation der Arztrolle).

\section{Kontext}

Das Phänomen „Identifikation“ ließ sich in keinem spezifischen Kontext verorten. Die Suche nach Vorbildern, mit denen sich die Studierenden identifizieren konnten, erfolgte zum Teil zufällig über Begegnungen mit Ärzten, z.B. im Rahmen von Praktika, zum Teil suchten die Studierenden jedoch auch gezielt nach Vorbildern (s.u. „Handlungsstrategien“). Erleichtert wurde es den Studierenden, Ärzte (und auch Kommilitonen) als Vorbilder zu finden, wenn diese offen ihre eigenen Motive und Einstellungen artikulierten (S05 103). 


\title{
Ursächliche Bedingungen
}

Identifikationen hingen u.a. vom Verhalten der Dozenten im hierarchischen Gefüge der Medizin ab. Die Studierenden erlebten Ärzte in hohen Positionen eher als Vorbilder, wenn sie sich ,auf Augenhöhe‘ begegneten und auf mögliche Privilegien allein aufgrund ihres Ranges verzichteten:

\begin{abstract}
„Also der ähm ... ja äh.. das sind so Banalitäten oder so aber //I:mhm// da waren da vier Computer und jeder sitzt an einem Computer und ähm.. dann hatte ich nen Patienten aufgenommen und musste das eingeben und dann steht er auf obwohl .. er hätte da ja sitzen bleiben können“ (S05 079).
\end{abstract}

Der Arzt erkannte die Arbeit der Studentin während eines Praktikums an, indem er ihr seinen Arbeitsplatz überließ. Besonders positiv empfanden die Studierenden es, wenn Dozenten Interesse an innen zeigten und auch „Persönliches“ von sich selbst erzählten. Ein Beispiel hierfür ist eine Professorin, die - im Gegensatz zu dem zuvor beschriebenen distanzierten Verhalten einiger Professoren - auf einer persönlichen Ebene mit Studierenden in Kontakt zu treten versuchte. Sie merkte sich Details über ihre Studenten und erkundigte sich nach ihrem Befinden, was als Ausnahme berichtet wurde. Dies veranlasste auch die Studierenden dazu, sich ihr anzuvertrauen:

„ja äh das ist die die kennst du auch die X-(Name der Professorin2) aus der X-(Name einer Abteilung) genau ja ich finde die hält ja schon total gute Vorträge //l:ja// und ähm .. genau dann meinte ich irgendwie zu ihr ja mir fehlt da ein Punkt und ich bin durchgefallen weil ich immer so äh also ich war halt super aufgeregt //l:mhm// und dann meinte sie nur äh hat auch so den Arm um die Schulter gelegt und meinte es ist nicht so schlimm das war bei ihr auch so" (S05 095).

Die beschriebene Situation zeugt von Mitgefühl und Vertrautheit. Zu einer Identifikation und größeren Nähe schien auch das Mitteilen von Erfahrungen mit Prüfungsangst beizutragen. Die Dozentin schloss ihre eigene Person in die Beziehung zu ihren Studierenden mit ein. In der Art, wie die Dozentin mit der "Schwäche“ der Studentin bezüglich ihrer Prüfungsangst umging, signalisierte sie zudem eine besondere Einstellung zum Thema Leistung und Leistungsdruck. Damit begegnete sie einem Bedürfnis nach Verständnis und Empathie für die eigene Situation im Studium, das mehrere Studierende äußerten (s. Kapitel 5.2.1: Orientierungshilfen durch Vorbilder: Die Rolle von Kommilitonen). Sich wie die oben beschriebene Professorin in Studierende hineinversetzen zu können, wurde als „Vorbildliche Eigenschaft" erachtet. Für Belange von Studierenden erreichbar zu sein und die Bereitschaft, die Studierenden 
Ergebnisse

zu unterstützen, waren ebenfalls Beispiele dafür, sie als ernst zu nehmendes Gegenüber zu behandeln.

Über die Identifikation mit Einzelpersonen gelang Studierenden offenbar auch die Identifikation mit der Fakultät oder dem Studium. Dabei spielte neben einer Identifikation mit Ärzten auch ein Zugehörigkeitsgefühl anderen Studierenden gegenüber eine wichtige Rolle.

Eigenschaften und Verhalten der beobachteten Ärzte, die für viele Studierende eine Vorbildfunktion hatten, waren die Wertschätzung des einzelnen Studierenden, Engagement bei der Vermittlung von Wissen, das Anliegen, den Studierenden das Lernen zu erleichtern und ein wohlwollender und respektvoller Umgang mit Studierenden und Patienten (vgl. u. a. S05 075, S11 103). Zusammenfassend beschrieben einige Studierende solche Eigenschaften als „,besondere Persönlichkeit“. Für die Bewertung einer Person als Vorbild nannten sie explizit als Voraussetzung, deren Persönlichkeit zu kennen:

„I: Ähm würdest du so jemand jetzt auch als Vorbild bezeichnen //P: Ja schon //I:mhm// also als Vorbild äh als Professor ne ich weiß nicht wie der als Mensch ist“" (S07 50).

Bei der Übernahme von Aufgaben in der Patientenversorgung „etwas zugetraut“ zu bekommen (S11 103), verstärkte den Eindruck der Studierenden, einbezogen zu sein, und die Identifikation mit der späteren Rolle als Ärzte.

Einige Studierende berichteten von unerwarteten, positiven Erfahrungen mit Professoren, die ihr vorgefertigtes Bild eines Professors und des Arztberufes korrigierten bzw. ergänzten. Das Bild eines Professors und damit verbundene Erwartungen von S07 wurden durch ein Zusammentreffen mit seinem Tisch-Dozenten revidiert; einen Professor im direkten Kontakt zu erleben, veränderte seine Einstellung zu „Professoren“ auch in Bezug auf sein eigenes Verhältnis zu ihnen (S07 18).

„ja war halt also relativ also entspannt ich hätte mir das nicht so also nicht so vorgestellt auch //l:hm// also ne lässig mit langen Haaren und Bart“ (S07 14).

Im direkten Kontakt zu Dozenten wurde es als positiv bewertet, dem einzelnen Studierenden Aufmerksamkeit zu schenken und trotz der großen Anzahl an Studierenden eine Beziehung zu innen aufzubauen (vgl. auch S05 095 und 103, S05 071):

„aber also als Verhaltensvorbild quasi wie man jemanden gegenübertritt so //l:mhm// da hab ich ja die X-(Name der Professorin) genannt //I: Ja genau// die irgendwie einen dann als nicht als kleinen Student irgendwie so unbedingt //l:hm// das Gefühl vermittelt dass man so irgend- 
Ergebnisse

wie nichts wert ist in dieser Maschinerie //I:mhm// was ja eigentlich de facto so ist dass man schleust ja die Studenten da durch hm und ähm (4) ja genau ja das sind dann so gesehen auch Vorbilder" (S07 55).

Solche Erfahrungen hatten nicht nur eine Rückwirkung auf die Einstellung der Studierenden zum angestrebten Beruf, sondern auch zum Studium. S03 sagte z.B., dass sie sich durch die Lehre einiger Dozenten „besser [im Studium] aufgehoben“ (S03 75) fühle.

Ein empathisches Verhalten von Ärzten gegenüber Patienten und Studierenden erleichterte ebenfalls eine Identifikation. Studentin S11 beschrieb eine Situation, in der sich die anwesenden Ärzte nicht nur dem Angehörigen einer verstorbenen Patientin, sondern auch ihr selbst gegenüber einfühlsam verhielten (S11 075). Den nächsten Satz ihrer Erzählung beginnt sie mit „wir als Ärzte“ (S11 075). Dass sie sich in ihrer Wahrnehmung mit einschließt und bereits der Gruppe der Ärzte zuordnet, lässt vermuten, dass sie sich an dieser Stelle mit dem Verhalten der beteiligten Ärzte und ihrer eigenen späteren Rolle als Ärztin identifizieren konnte. Als prägend erscheint die geschilderte Situation, da die Studentin hier unmittelbar am Vorbild lernen konnte.

In der Gesamtschau ergaben die beobachteten Situationen und geschilderten Eigenschaften für die Studierenden ein positives Bild der Rolle eines Arztes. Erleichtert wurde eine Identifikation mit Ärzten durch das Beobachten von angemessenem ärztlichen Verhalten und durch die Vermittlung positiver Aspekte des Berufes (vgl. S16 064).

\section{Intervenierende Bedingungen}

Besonders hohe Erwartungen an Dozenten waren ein deutlicher Hinweis auf die Suche der Studierenden nach Identifikationsobjekten, erschwerten es innen aber zugleich, sich mit Dozenten zu identifizieren und sie als Vorbilder anzuerkennen (im Kapitel 5.2.4: Auseinandersetzung mit Idealen der ärztlichen Profession wurden hohe Erwartungen ebenfalls als intervenierende Bedingung beschrieben). Wenn Dozenten die Erwartungen der Studierenden nicht in ihrer Gesamtheit erfültten, wurden sie schnell als Negativbeispiele wahrgenommen.

Negative Erfahrungen im Studium und Unzufriedenheit mit der Lehre schienen die wichtigsten Faktoren zu sein, die eine Identifikation mit dem Studium erschwerten 
Ergebnisse

oder sogar verhinderten. Studentin S12 erzählte von mehreren negativen Erfahrungen im Studium und gab an, kein Vorbild innerhalb der Universität zu haben:

„und sonst find ich den größten Teil von der Lehre .. scheiße //I:mhm// und das meiste ist schon irgendwie interessant aber ich hab nicht das Gefühl dass es irgendwie bei mir so andockt“ (S12 42).

Innerhalb der medizinischen Fakultät schien sich S12 mit keiner Person zu identifizieren. Die Kenntnis der Persönlichkeit des Gegenübers schien eine Rolle für Identifikation und Vorbildfunktion zu spielen, jedoch nicht Voraussetzung dafür zu sein. Die Studierenden lobten zwar ein Interesse der Dozenten an ihrer Person, genauso wurde jedoch ihr „Auftreten“ aus der Ferne bewundert, ohne dass ein direkter Kontakt bestand. Eine gewisse Distanz zur Position und dem Wissensstand einer Person schien zugleich eine weitere Voraussetzung für eine Vorbildfunktion zu sein (vgl. S08 09). Gerade ein Abstand zur Situation der Studierenden verlieh solchen Vorbildern Autorität. Sie wurden besonders aufgrund von Wissens- und Erfahrungsunterschieden anerkannt.

\section{Handlungsstrategien}

Trotz wahrgenommener Distanz zu ihren Dozenten setzten sich die Studierenden mit ihren Einstellungen und ihrem Verhalten auseinander, indem sie z.B. versuchten, Rückschlüsse aus deren Verhalten auf ihre Persönlichkeit oder zu Grunde liegenden Motiven zu ziehen (vgl. S05 031). S05 fiel z.B. auf, dass zwei in ihrer Wahrnehmung vorbildliche Dozenten befreundet waren. Dies brachte sie in einen Zusammenhang damit, dass beide über "menschliches Vermögen“ verfügten (S05 067).

Die Studierenden suchten nach ärztlichen Vorbildern, mit denen Gemeinsamkeiten oder Ähnlichkeiten bestanden. Student S13 hatte beispielsweise ein besonderes Interesse an Disziplinen der Medizin, in denen es um ein schnelles Handeln und eine Belastbarkeit in Stresssituationen geht (S13 108). Er bewunderte einen Unfallchirurg für seine Kompetenz und beschrieb inn mit ähnlicher Wortwahl wie seine persönlichen Ziele im Studium und Beruf (z.B. ein umfangreiches Allgemeinwissen und schnelles Handlungsvermögen in Notallsituationen, vgl. S13 003 und S13 108). Er suchte Kontakt zu dem Arzt, da er den Anforderungen des Studenten gerecht wurde und Ziele in seinem Berufsalltag erfüllte, in denen der Student sich wiedererkannte. Es ging also nicht nur um vorbildliche Eigenschaften eines Arztes. Wichtig war den Studierenden auch, ob sie für sich einen Bezug zu diesen Eigenschaften finden 
konnten, ob sie einen Anteil daran hatten oder sogar sich selbst oder ihre Zukunft darin sehen konnten. S05 berichtete von einer Dozentin, die im Seminar ihre Meinung über Verhütungsmittel mit den Studierenden teilte (S05 103). Gleichzeitig versuchte sie auch, die "Studenten mit ihrer Begeisterung [für ihr Fach] anzustecken“ (S05 111). Die Studentin entschied darauf hin, ihre Doktorarbeit bei ihr zu schreiben (S05 027, S05 111). Genau dafür, nämlich in eine Beziehung zwischen Vorbild und Studierendem zu treten, war ein gegenseitiges Interesse Voraussetzung.

\section{Konsequenzen}

War eine Identifikation mit Ärzten nicht möglich, gab es also keine Vorbilder, mangelte es auch an einer Identifikation mit der Arztrolle. S06 fühlte sich im Praktikum auf die Unterstützung des Pflegepersonals angewiesen, da ihr von Seiten der Ärzte kein Interesse entgegengebracht wurde, so dass sie sich auch eher mit den Pflegekräften identifizierte:

„hab ich einen Monat Pflegepraktikum gemacht //l:mhm// und da hatte ich zum Beispiel also das ist ja im Pflegepraktikum auch so gedacht dass man //l:mhm// ja mit dem Pflegepersonal mit den Schwestern und so mitläuft und diese Seite kennen lernt aber da hab ich zum Beispiel gemerkt dass so die die ärztliche Seite mir die teilweise ganz fern war //I:mhm// also dass die sich auch gar nicht dafür interessiert haben was ich mache //l:mhm// oder mich gefragt haben ob ich irgendwo mitgehen will und was sehen will .. obwohl die wussten dass ich Medizin studier und ähm //l:ja// dann kann ich mich zum Beispiel erinnern dass ja irgendwie einschneidend war wie gut ich mich da mit dem Pflegepersonal verstanden habe“ (S06 03).

Fanden die Studierenden jedoch Ärzte, mit denen sie sich identifizierten, fühlten sie sich auch besser auf die zukünftige Rolle als Ärzte vorbereitet. S05 beschrieb beispielsweise, wie sie über die Beobachtung von Ärzten den Umgang mit starken Emotionen im Patientenkontakt gelernt habe:

„Das hat einem zumindest so für später glaub ich ein bisschen Sicherheit gegeben weil ich jetzt zumindest besser weiß wie ich mit solchen Situationen umgehen kann" (S05 131).

Ob sich die Studierenden auf die Rolle als angehende Ärzte vorbereitet fühlten, hing von den im Studium gemachten Erfahrungen ab. Teilweise führte die Auseinandersetzung mit dem beobachteten Verhalten von Ärzten auch dazu, dass sich Studierende davon distanzierten. Eine andere mögliche Konsequenz bestand darin, eigene Idealvorstellungen zu überdenken oder anzupassen, analog der Auseinandersetzung mit ärztlichen Idealen anhand real erlebter Situationen mit Ärzten (s. Kapitel 5.2.4: Auseinandersetzung mit Idealen der ärztlichen Profession). 
Abbildung 5 fasst die Ergebnisse zum Thema „Identifikation mit Ärzten, Studium und Beruf“ im Sinne des Kodier-Paradigmas zusammen:

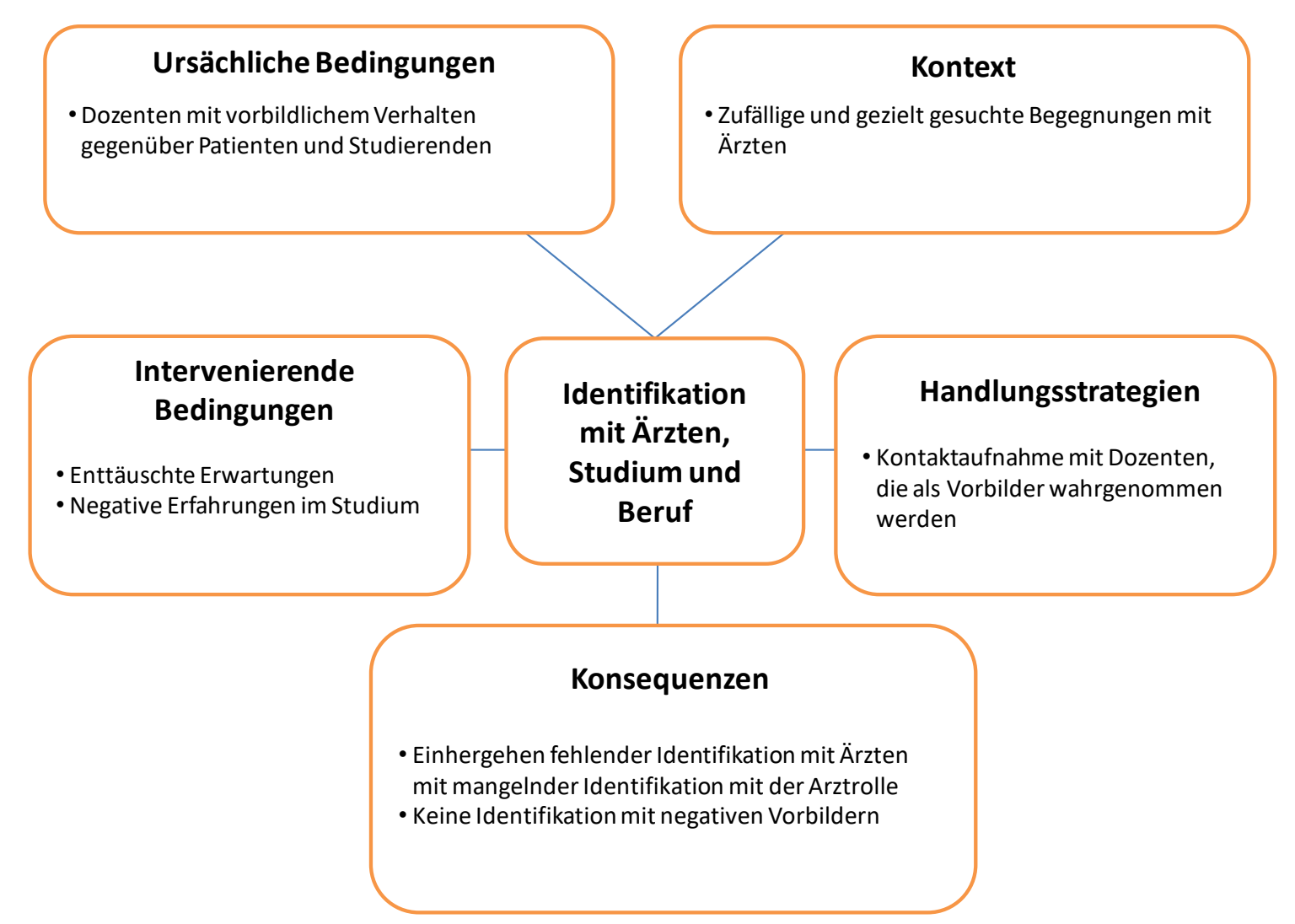

Abbildung 5: Kodier-Paradigma zum Thema „Identifikation mit Ärzten, Studium und Beruf“.

\subsubsection{Auseinandersetzung mit Idealen der ärztlichen Profession}

\section{Das Phänomen}

Die Übernahme von Idealen der ärztlichen Profession schien nicht nur davon abzuhängen, was Mitglieder der Fakultät Studierenden zu vermitteln versuchten, sondern auch davon, was Studierende für wichtig erachteten und zu übernehmen bereit waren. Da innen Empathie in der Patientenversorgung häufig sehr wichtig war, taugten Dozenten zumeist nur dann als Ideal, wenn auch ihr Verhalten Patienten und Studierenden gegenüber mit diesem Ideal vereinbar war (vgl. S13 007, S11 095). Sie berichteten, Ärzte höheren Ranges vermittelten innen zwar, „was" Studierende lernen sollten, unterstützten sie jedoch nicht dabei zu lernen, „wie“ sie sich als Arzt verhalten sollten (s.u.). 
Ergebnisse

Erwartete Ideale und das mitgebrachte Verständnis idealen ärztlichen Verhaltens konfligierten oft mit dem beobachteten Verhalten von Ärzten. Ein Ideal, das viele Studierende beschäftigte, war die "Menschlichkeit" von Ärzten, die als zentraler Wert eines vorbildlichen ärztlichen Verhaltens erachtet wurde (vgl. S05 003 und 067, S07 04). Der Begriff "menschlich" wurde dabei in unterschiedlichen Zusammenhängen verwendet und mit verschiedenen Bedeutungen verknüpft (s.u.).

\section{Kontext}

Neben einer erlebten Diskrepanz zwischen mitgebrachten Idealvorstellungen und Realität wurde bereits im Studium die Umsetzung eigener Wertvorstellungen als schwierig wahrgenommen. Speziell während überfordernder Situationen im Studium schienen die Studierenden besorgt darüber, ob Werte wie Menschlichkeit gewahrt und ausgeübt werden konnten. Ein häufig genanntes Beispiel einer als "grenzüberschreitend" wahrgenommenen Situation war der Anatomiekurs. Das Präparieren von Leichen wurde bereits als "aufreibend“ erwartet (S04 007), als "wichtiges Erlebnis“ und „bedrückend“ erinnert (S04 003 und 055) und brachte einige Studierende an die Grenze dessen, was für sie aushaltbar war (vgl. S04 055, S15 027). S04 äußerte, es sei wichtig, „dass man sich noch darüber wundert dass man sich so gewöhnt an jemandem herum[zu]schneiden“ (S04 055). Die Angst, sich an „belastende Situationen" zu gewöhnen, beschrieben mehrere Studierende ( $v g l . ~ S 04$ 055, S08 40 und 10). Da sie Ärzte im Krankenhaus teilweise als „unmenschlich“ (vgl. S05 031) erlebten und den Eindruck hatten, Dozenten würden sich häufig nicht von ihrer „menschlichen Seite“ zeigen, hatten Studierende auch die Sorge, sie könnten ihre eigene „Menschlichkeit" während ihrer Ausbildung und in der Berufsrealität verlieren. Erlebnisse im Studium schienen die Befürchtung der Studierenden zu verstärken, es könne im Beruf zu einem „Werteverfall“ kommen. Student S04 kritisierte das Auftreten eines Professors, der zwar fachlich kompetent sei, dem jedoch ein "herzlicher Umgang" fehle (vgl. S04 007). Student S08 machte sich Gedanken über die Auswirkungen einer wissenschaftlichen Karriere auf die Persönlichkeit von Ärzten. Er selbst bekundete Interesse an der Forschung, schien jedoch eine gewisse Gefahr darin zu sehen, mit der Zeit Ähnlichkeiten mit beobachteten Professoren zu entwickeln:

„viele Professoren haben so $\mathrm{n}$ bisschen den find ich verlieren so den Kontakt zu den //I:mhm// den Studenten und wirklich ähm nicht nur so zu den Studenten sondern überhaupt zu ihrer Umwelt //I: ((lacht))// und die wenigen die das irgendwie nicht so sehr tun .. die sind dann ir- 
Ergebnisse

gendwie immer gleich ähm beeindruckende Persönlichkeiten weil die irgendwie noch'n bisschen was mehr haben als nur ihr Fachwissen“ (S08 10).

Es beunruhigte inn, dass er bereits ein ähnliches Verhalten bei sich selbst beobachtete:

„Die Zeit war natürlich sehr intensiv es war direkt vorm Physikum .. und ähm eigentlich muss ich sagen hab ich in der Zeit eigentlich wenig Kontakt zur Außenwelt gehabt //l:mhm// meistens halt weil ich zuhause gewesen bin .. es warn halt wenige Menschen zu denen ich Kontakt hatte" (S08 40).

Den Begriff „menschlich“ besetzten Studierende mit unterschiedlichen Bedeutungen: Empathie, Authentizität, Ganzheitlichkeit, Gleichberechtigung, aber auch „Menschlichkeit" in Bezug auf das Auftreten im Umgang mit Patienten. S14 bezeichnete die altruistische Bereitschaft eines Arztes, hohe Arbeitsbelastungen und Anforderungen für Patienten zu ertragen als „menschlich“ (vgl. S14 083). Viele der Studierenden bezogen „menschliches“ Verhalten auf einen empathischen Patientenumgang, einige auch auf einen empathischen Umgang mit Studierenden. Dies war vor allem im Zusammenhang mit überfordernden Situationen der Fall, zu denen auch erste Erfahrungen im Krankenhaus zählten, verbunden mit einem Bedürfnis nach Unterstützung:

„ja die bereit sind einem was beizubringen die sich in die Situation von einem selber reinversetzen können und sich zurückerinnern wie war es früher bei mir“ (S03 11).

Nicht nur in diesem Punkt wurde ein ähnliches Verhalten im Umgang mit Studierenden und mit Patienten als vorbildlich erachtet. Unter einer „empathischen“ Patientenversorgung verstanden die Studierenden z.B. eine einfühlsame Art zu kommunizieren und die Fähigkeit, das eigene Verhalten als Arzt auf Gesprächssituationen abzustimmen (vgl. S04 075 und 079). Als „menschlich“ erachteten sie eine Betreuung von Patienten, die über die medizinisch-körperliche Versorgung hinausging und gerade auch emotional bedeutsame Anliegen von Patienten berücksichtigt (wie beispielsweise die Organisation eines Kindergeburtstages auf einer Palliativstation, S04 075). In diesem Zusammenhang wurde von den Studenten eine „ganzheitliche“ Patientenversorgung als menschlich bezeichnet, die die Intention, dem Patienten wirklich zu helfen (S11 091) und seine „Rundum-Versorgung“ mit einschloss (vgl. S05 04, S11 063):

„hat denen halt zugehört und halt auch wirklich also der war wirklich dran interessiert also der hat halt auch als Fachgebiet eben Rheuma gehabt //I:mhm// was ja auch nicht so einfach ist und da kamen halt Patienten auch an die irgendwie seit Ewigkeiten irgendwo Schmerzen hat- 
Ergebnisse

ten keiner hatte bisher was gefunden und er hat sich richtig hingesetzt und hat richtig danach gesucht so //l:mhm// und war richtig daran interessiert denen auch zu helfen“ (S11 091).

Als Arzt „menschlich zu sein“ brachten die Studierenden jedoch auch in einen Zusammenhang damit, authentisch aufzutreten (vgl. S04 071). Neben einem einfühlsamen und gewissenhaften Auftreten von Ärzten (S04 074) wurde es auch bewundert, als Arzt informell und offen mit der eigenen Meinung umzugehen. So wertete es Student S04 als „angenehm“, als Arzt auch mal zu fluchen (S04 011und 074).

Insgesamt erscheinen die Erwartungen der Studierenden an eine menschliche Patientenversorgung komplex und teilweise widersprüchlich. Sie erwarteten von Ärzten einen gleichberechtigten Umgang mit Patienten, jedoch gleichermaßen auch, innen Halt zu geben und Verantwortung für sie zu übernehmen:

„wie sie mit Patienten ähm oder für den Patienten ne Sicherheit gegeben haben dass sie ne medizinische Kontrolle über sie haben und dass sie sich auf die Ärztinnen verlassen können //I:mhm// und das waren für mich irgendwie so Vorbildfunktionen" (S05 003).

\section{Ursächliche Bedingungen}

Die Auseinandersetzung mit dem Ideal eines „menschlichen“ Verhaltens von Ärzten war zum einen für die Studierenden vor dem Hintergrund relevant, dass sie sich auf die zukünftige Arztrolle vorbereiteten. Die Komplexität medizinischer Themen und möglicher Haltungen dazu schien durch den Austausch mit anderen Mitgliedern der Fakultät noch größer zu werden und die Studierenden dazu zu veranlassen, Wertevorstellungen zu hinterfragen:

„Naja was ich zumindest faszinierend finde dass zwar alle das Gleiche studieren aber doch so unterschiedlich sind ähm //l:mhm// auch in ihren Einstellungen //I:mhm// aber ähm in es macht's ja vielleicht nicht unbedingt leichter dann seinen eigenen Standpunkt zu finden sei es jetzt medizinisch oder //I:mhm// alles was so zusammenkommt auch menschlich gesehen [...] verschiedene Möglichkeiten.. ähm //I:mhm// eigentlich auch die ganzen Einstellungen zu hinterfragen eben gerade wie steh ich zu manchen medizinischen Sachen //I:mhm// sei es Sterbehilfe sei es irgendwie ähm.. Transplantation“ (S05 023).

Zum anderen galt dem Thema "Menschlichkeit" auch Interesse, da angestrebt wurde, auch im Kontext neuer studienbedingter Anforderungen und trotz beruflicher Arbeitsbedingungen menschlich zu bleiben. Die Studierenden wünschten sich wie S03 im Rahmen von Seminaren "man selber" sein zu können (S03 51). Dozenten, die sich den Studierenden „als Mensch“ offenbarten, bewiesen nicht nur, dass dies möglich war (vgl. S03 47), sondern gewährten ihnen auch direkte Einblicke in ihren persönli- 
Ergebnisse

chen Umgang mit Idealen im Beruf. Ihr vorgelebtes Verhalten und Einstellungen zu vergleichen, war bereits Teil der Auseinandersetzung der Studierenden mit Idealen. Auch das Verhalten von Ärzten den Studierenden gegenüber spielte eine Rolle dabei, ob ihre Erwartungen an „Vorbildliches ärztliches Verhalten“ erfüllt wurden. Dieses wurde von den Studierenden häufig attestiert, wenn beobachtete Ärzte ihre Erwartungen im Umgang mit ihnen übertrafen oder sie positiv überraschten. Jedoch führten auch Situationen dazu, sich mit Idealen der ärztlichen Profession und ihren Vorbildern auseinanderzusetzen, in denen Ärzte den Erwartungen der Studenten nicht entsprachen und eine Diskrepanz zwischen Ideal und Realität erlebt wurde (s. u.).

Insgesamt schien die Vermittlung von Idealen durch das eigene Beispiel am wirkungsvollsten zu sein. Das Demonstrieren eines von Studierenden geforderten Verhaltens Patienten gegenüber erschien glaubhaft, wenn dies mit ihrer persönlichen Erfahrung mit Dozenten vereinbar war. „Auf einer menschlichen Ebene“ respektiert zu werden erreichte die von S05 beschriebene Dozentin z.B., indem sie den Studierenden am eigenen Beispiel demonstrierte, was ein respektvoller Umgang für sie bedeutete (vgl. Kapitel 5.2.3: Identifikation mit Ärzten, Studium und Beruf). Sich als Student respektiert zu fühlen war eine unmittelbare Erfahrung, die ihnen ,am eigenen Leib‘ zeigte, wie sich solch ein Verhalten auch auf Patienten auswirken könnte.

\section{Intervenierende Bedingungen}

Konkurrierende oder widersprüchliche Idealvorstellungen von ärztlich korrektem Verhalten erschwerten die Suche der Studierenden nach Richtlinien oder Verhaltensmaßstäben: So bewunderten und erwarteten sie ein engagiertes, altruistisches Verhalten von Studenten und Ärzten (vgl. u.a. S11 09), schätzten auf der anderen Seite jedoch ebenfalls Authentizität von Ärzten und die Beobachtung einer Work-LifeBalance (vgl. u.a. S14 011 und 083, S05 095, S08 15). Dies zeigte auch die Uneinigkeit der Studierenden über die Definition „vorbildlichen Verhaltens“ von Ärzten. Die Komplexität der Thematik wird dadurch größer, dass unterschiedliche Werte miteinander konkurrierten. In Bezug auf eine gleichberechtigte Beziehung zu Patienten beschrieb S05 das Verhalten einer Ärztin:

„,naja dann kann ich ja mal jetzt die gerade die Situation erzählen zumindest die fand ich auch ziemlich ähm... ja.. vorbildmäßig also //l:mhm// eben diese Ärztin die ähm sehr.. menschlich mit den Patienten umging //I:mhm// sie gleichzeitig aber nicht bemitleidet hat oder sie irgendwie als krank hingestellt hat sondern //l:mhm// sie sehr respektvoll behandelt hat" (S05 003). 
Ergebnisse

Patienten als „Kranke hinzustellen“ und sie zu „bemitleiden“, war für S05 negativ besetzt. Sie äußerte im Weiteren die Annahme, Ärzte könnten durch ihr Verhalten Patienten stärker „in ihre Krankheit hineintreiben“ (S05 035). S05 ging sogar soweit zu sagen, Ärzte dürften Menschen „nicht als Patienten behandeln“, sondern

„als gleichwertigen Partner [...] betrachtet haben und ähm gleichzeitig innen aber auch so ne Sicherheit gegeben haben“ (S05 003).

Die Erwartung der Studentin konkurriert - vielleicht unbewusst - mit ihrer Vorstellung, Patienten nicht als untergeordnet zu behandeln (vgl. S05 031), „Medizinische Kontrolle" (S05 003) liege allein in der Verantwortung des Arztes, und ein Arzt müsse Verantwortung für seine Patienten übernehmen und ihnen Sicherheit geben (vgl. S05 031). Hier also wird die Arzt-Patienten-Beziehung nicht als gleichberechtigt charakterisiert.

Gleichzeitig Gleichberechtigung und die Verantwortung für das Wohlergehen des Patienten zu gewährleisten, ist dabei nur eine Herausforderung in der Verwirklichung einer menschlichen Patientenversorgung. Die Erwartungen der Studierenden diesbezüglich wirkten insgesamt komplex, anspruchsvoll und ambivalent.

Unterschiede schienen nicht nur in den Definitionen einer „idealen Patientenversorgung" zu bestehen, sondern auch in deren Gewichtung. Die Studierenden hatten den Eindruck, ihre Dozenten würden Faktenwissen einen besonders hohen Wert beimessen (vgl. S05 011). Auch wenn Studierende autoritäres Verhalten von Dozenten im Allgemeinen ablehnten, erachteten sie deren Leistung und Rolle als überlegen und ordneten sich unter. S08 beschrieb sein Vorbild mit Ehrfurcht. Der Student wirkte überzeugt davon, sein Betreuer der Doktorarbeit habe ein so hohes Niveau in seinem Fach erreicht, dass er ihm zwar nacheifern, jedoch nie Ähnliches erreichen könne.

S05 lobte einen Arzt mit den Worten, dass er „selbst mit den Famulanten total kollegial“ umging (S05 007). Die Wortwahl („selbst“) spricht für ein Denken in Hierarchien. Dies stand in direktem Zusammenhang mit ihrem Wissensstand. Sie hatte den Eindruck, nur dann von Ärzten höheren Ranges respektiert zu werden, wenn sie gute Leistungen zeigte:

„dann waren das Begegnungen mit Dozenten die einen vielleicht in dem Moment richtig //I:mhm// gezeigt haben du bist noch gar nicht du kannst noch nichts und du kriegst noch keinen Respekt“ (S05 011). 
Ergebnisse

Da die meisten Dozenten - in der Perspektive der Studierenden - ihre Rolle als Vermittler von Wissen sahen, verstärkten sie das Gefühl der Studierenden, Leistung erbringen zu müssen, um akzeptiert zu werden. Die Studierenden schienen dabei das Relevanzsystem ihrer Dozenten zu übernehmen: S05 betonte mehrfach, dass es für sie wichtig sei, von Dozenten nicht als unwissend "hingestellt" zu werden (S05 095, 063 und 011). Andere Studierende berichteten, wie Dozenten über ihre Position und ihr größeres Wissen sowie ihre Erfahrung ihre Überlegenheit zeigten. Ein Dozent wurde in diesem Zusammenhang als jemand beschrieben, der Freude daran empfindet, Wissenslücken in Prüfungen (oder auch in Seminaren und Kursen) aufzudecken und den Studenten das "Gefühl (zu) geben, dass sie das alles nicht können" (S04 051). Die Studierenden seien daraufhin „in Tränen ausgebrochen“ (S04 0 51). S07 lobte seinen Professor dafür, dass er seine Machtposition nicht ausnutzte:

„der hat uns kann ein ja ziemlich unter Druck setzen irgendwie bei dem Präpkurs //l:mhm// und ähm das hat der nicht gemacht und das rechne ich dem halt hoch an dass er äh //I:Ja// das auch irgendwie dann noch also menschlich war ///:mhm// und dann einfach so ne heute sind wir so weit gekommen und macht vielleicht nächstes Mal bisschen schneller aber nicht irgendwie total unter Druck oder dass man Nachsitzen musste genau weil das schon auch insgesamt eine belastende Situation ist ähm und ähm da ist es halt glaub ich vor allem Dingen halt wichtig dass man jemand hat der der ne ruhige Ausstrahlung hat" (S07 14).

Dass von S07 angenommen wurde, die Dozenten hätten die Möglichkeit, die Studierenden auch "nachsitzen“ zu lassen, impliziert, dass solche disziplinarischen Maßnahmen nicht ausgeschlossen waren. Insgesamt wurde es als Ausnahme wahrgenommen, als Student von Dozenten aufgebaut und gefördert zu werden (S05 063). Dies hatte zur Folge, dass die Studierenden gehemmt wirkten, Wissenslücken in der Gegenwart von Dozenten offen zu legen. Wenn keine Vertrauensbasis zu Dozenten höheren Ranges bestand, stellten Studierende studentischen Hilfskräften Fragen z.B. bevorzugt, wenn der Dozent nicht vor Ort war (S12 19). Die Aussage von S07, Professoren seien „unantastbar“ (S07 18) spiegelt die Vorstellung, Professoren seien in ihrem Fachwissen unerreichbar.

Auch die Studierenden erachteten Faktenwissen für essentiell (vgl. S11 119). Wissen schien für sie jedoch nur eine wichtige Eigenschaft zu sein und nicht wichtiger als z.B. persönliche Einstellungen (vgl. u.a. S06 83, S05 103 und 139, S04 003 und 031 , S08 03). Die Studierenden äußerten Bedenken, andere wesentliche Lerninhalte, die über Fachwissen hinausgehen wie z.B. Empathie zu entwickeln, könnten vernach- 
Ergebnisse

lässigt werden (vgl. S08 10, S03 47). Sie lobten vor allem Ärzte, die über beides Fachwissen und Empathie - verfügten (vgl. S05 055 und 095, S15 051). Ausschließlich fachliche Kompetenz genügte nicht, um als Vorbild bezeichnet zu werden (vgl. u.a. S13 052, S07 04). S08 sagte über Professoren, die „mehr haben als nur ihr Fachwissen“, diese seien „dann immer gleich beeindruckende Persönlichkeiten“ (S08 10). Als Arzt eine „besondere Persönlichkeit“ zu haben, wurde von vielen Studierenden als wichtig erachtet:

„ansonsten was ich auch also von Lehrenden also auch immer cool fand oder was mich beeindruckt hat war wenn du gemerkt hast da steht irgendwie $\mathrm{n}$ Charakter da vorne //l:Ja// ne jemand der unverwechselbar ist" (S07 04).

Eine „besondere Persönlichkeit“ wurde ebenfalls als Ideal angesehen und mit dem Thema „als Arzt menschlich zu sein“ in Verbindung gebracht (vgl. S05 027). Dozenten, die den Studierenden persönliche Seiten zeigten, vermittelten den Studierenden damit auch, dass Wissen nicht alleiniger Maßstab sei.

Häufig schien es den Studierenden im Krankenhaus während Praktika an positiven Vorbildern zu mangeln. Nicht nur wurden Kontakte mit Patienten im Rahmen erster klinischer Praktika teilweise nicht betreut (S15 007 und 055), auch hatten Studierende manchmal den Eindruck, das Stationspersonal zu behindern und aufgrund der eignen Unwissenheit zu „nerven“ (S13 053). Das Gefühl, während Praktika im Krankenhaus als Student „keinen Platz" zu haben, beschrieb auch S08, was inn auch davon abhielt, sich an den Stationsärzten zu orientieren und direkt am Vorbild zu lernen. Wie in einem von $\mathrm{S} 15$ berichteten Beispiel hatte das Krankenhauspersonal jedoch auch durch „passives Verhalten“ eine Vorbildfunktion für die Studierenden im Umgang mit Patienten, selbst wenn dies nicht beabsichtigt wurde: Im Rahmen eines Praktikums beobachtete die Studentin, dass das Stationspersonal den Kontakt zu einem krankheitsbedingt „schwierigen“ Patienten meidet (S15 007 und 055). An dieser Stelle scheint ihr ein Vorbild, das einen menschlichen Umgang mit solchen Patienten vorlebt, zu fehlen.

Hinderlich bei der Suche nach Idealen erschienen hohe Erwartungen der Studierenden an ärztliches Verhalten. Sie beschrieben die beobachteten Ärzte mit stark wertenden Ausdrücken (S04 007, S04 052); S05 z.B. sagte über einen Arzt, er sei „fehl am Platz" und man dürfe es als Arzt „auf keinen Fall machen“ wie er (S05 031 und 003). An Ärzte legten Studierende höhere Maßstäbe als an die Normalbevölkerung. 
Ergebnisse

S05 äußerte, ein Arzt, der seinen Patienten nicht hilft, habe in ihren Augen seinen Beruf verfehlt und sei ein „schlechtes Beispiel“. Das Verhalten des Arztes wurde sogar als „unmenschlich“ beschrieben (s. S. 39), obwohl es sich auch um eine ,natürliche oder menschliche‘ Reaktion handeln könnte. Die hohen Erwartungen erschwerten es den Studierenden, sich an ärztlichen Vorbildern zu orientieren, da nur noch wenige Ärzte überhaupt ihre Kriterien für ein Vorbild erfüllten. Wirkliche Vorbilder mussten über herausragende Fähigkeiten, wie z.B. exzellente klinische Kompetenzen verfügen (vgl. S05 047).

\section{Handlungsstrategien}

Die Studierenden setzten sich mit dem von ihnen beobachteten Verhalten auseinander, indem sie es verglichen und bewerteten. S03 beschrieb Vorbilder und deren Funktion in diesem Zusammenhang als „Personen von denen man sich so ne Scheibe abschneiden kann“ (vgl. S03 47). Umgekehrt fördern negative Beispiele die Entscheidung, nicht wie die erlebten Ärzte werden oder handeln zu wollen (vgl. S11 083). Für das Problem, dass eine Person häufig nicht alle Erwartungen der Studierenden gegenüber ärztlich korrektem Verhalten erfüllte, fand S07 folgende Lösungsstrategie:

„I: Und gab's noch andere //P: Vorbilder// P: Für [...] mich war (es) immer so dass ich unterschiedliche Leute also eben die unterschiedlich guten Eigenschaften von denen so als Vorbild //I:mhm// genommen hab aber nie irgendwie ne Gesamtperson irgendwie" (S07 53).

Sich an Teilaspekten von Personen zu orientieren, löste das Dilemma, dass immer wieder Aspekte an zuvor als vorbildlich identifizierten Ärzten gefunden wurden, die diesem Bild nicht entsprachen (vgl. S05 115).

\section{Konsequenzen}

Die Studierenden hatten die Möglichkeit, am Vorbild zu beobachten, wie Ideale der Profession, z.B. ein menschlicher Patientenumgang im Berufsalltag praktiziert wurden. Dabei nahmen einige Dozenten die Rolle von Vorbildern, andere von „AntiVorbildern“ ein. Ein Mangel an positiven Vorbildern erschwerte oft auch eine direkte Wertevermittlung. Umgekehrt wirkte das Verhalten von Anti-Vorbildern manchmal prägend. Die Studierenden hatten gewissermaßen die Aufgabe, eigene professionelle Einstellungen zum Beruf zu entwickeln und konkurrierende Wertevorstellungen zu vereinigen. Um für Studierende Vorbild zu werden, spielte das ärztliche Verhalten 
den Studierenden selbst und Patienten gegenüber eine wichtige Rolle. In beiden Fällen war es Studierenden im Wesentlichen wichtig, wenn sich Ärzte nicht „überlegen“, sondern gleichberechtigt und menschlich verhielten (vgl. u.a. S05 031).

Ideale am direkten Beispiel anhand von realem Verhalten erleben zu können, schien einen nachhaltigeren Eindruck bei den Studierenden zu hinterlassen als Worte oder Ermahnungen. Dies setzte voraus, dass Dozenten Studierende in ihre Tätigkeit einbezogen. Positiv überraschende Erfahrungen mit Dozenten und ihr Bemühen, die bestehende Distanz zu innen zu überwinden, veränderte auch das Verhalten der Studierenden. Studentin S03 erzählte von einem mündlichen Testat, zu dem sie zu spät kam. Ihre Befürchtung, der Prüfer würde dies strafen, wurde nicht bestätigt. Vielmehr zeigte er Nachsicht und hielt eine faire Prüfung mit ihr ab. Positiv von dem Verhalten des Prüfers überrascht zu sein, veranlasste sie zu einer persönlichen Geste, ein Geschenk.

Die Studierenden orientierten sich auch in Bezug auf "Nähe“ am Verhalten der Dozenten (vgl. Kapitel 5.2.2: Orientierungshilfen durch Vorbilder: Die Rolle von Lehrenden im Studium und für den Beruf) und sahen dies als Maßstab erwünschten Verhaltens (vgl. Kapitel 5.2.5: Rolleneinübung: Auseinandersetzung mit der Rolle als Medizinstudent/in und Antizipation der Arztrolle). Entstand ein direkter Kontakt, war ein Vorbild dann weniger ein Inbild eines „perfekten Arztes“, gleichsam unerreichbar; vielmehr wurde die betreffende Person zu einem „direkten“ Vorbild, das in einer von „Nähe“ gekennzeichneten Beziehung erlebt wurde. Sie überzeugte durch eine „besondere" Persönlichkeit und vermittelte Werte durch ihr Handeln (vgl. u.a. S08 10, S07 28-29). S07 bezeichnete eine Professorin als „Verhaltensvorbild“ dafür, „wie man jemandem gegenübertritt" (S07 55) in der unmittelbaren Interaktion.

"Nähe“ in der Beziehung zu Dozenten lief jedoch nicht immer darauf hinaus, ein Vorbild zu finden. So setzten sich die Studierenden auch mit der Bedeutung von Funktionen und Positionen innerhalb der medizinischen Hierarchie auseinander. Die Möglichkeit, einen Professor, der zuvor in einer Lehrveranstaltung bewundert wurde, kennen zu lernen, nahm z.B. einigen Studierenden das Bild von „Vollkommenheit“, senkte ihre Bewunderung für Leistung und Titel (S07 18: „so ne Ansammlung von Titeln“).

Trotzdem spielten die Position und Hierarchieunterschiede eine Rolle bei der Vorbildwirkung. Lehnten Studierende Autoritäten und Hierarchien einerseits ab, waren 
Ergebnisse

sie andererseits aber (zumindest für einen Teil der Studierenden) geradezu eine Bedingung für die Vorbildwirkung. Studierende bewunderten Dozenten aufgrund ihres Wissensvorsprungs und genossen es auch, von ihnen aufgrund der eigenen guten Leistung gelobt zu werden. Auf der anderen Seite kritisierten sie Dozenten in ihrem Verhalten, Hierarchieunterschiede zu betonen und forderten, von innen gleichwertig behandelt zu werden. Die Distanz zur Position der Dozenten und zu ihrem Wissensstand erscheint teilweise wie ein Grund zur Bewunderung. Gleichzeitig spornte dies aber nicht dazu an, innen nachzueifern, da das von ihnen verkörperte Ziel für die Studierenden zu weit entfernt wirkte. S07 überlegte, ob ein Professor für inn überhaupt ein Vorbild sei, da er sich nicht sicher war, ob er diese Position erreichen könne und auch, ob er sie erreichen wolle (vgl. S07 55).

Die Auseinandersetzung mit Vorbildern führte manchmal zu Veränderungen der Wertevorstellungen der Studierenden und ihren Einstellungen zum Beruf. So deutete sich bei S14 eine Veränderung ihrer Vorstellung eines „idealen Arztes“ an: „Früher wollte ich auch mal so ne Ärztin [wie der Arzt in X-(Name eines Landes)] werden" (S14 082). Sie beschrieb, dass man dort als Arzt "wirklich helfen kann" (S14 087), und wie die Tätigkeit als Arzt „einen Zugang zu den Menschen" (S14 091) verschaffe. Die Studentin erkannte jedoch bereits zum Zeitpunkt des Interviews (6. Semester), dass ihre früheren Vorstellungen vom Arztberuf vom medizinischen Alltag in Deutschland abwichen. Sie war durch ihre Erfahrungen im Studium zu der Überzeugung gekommen, solch eine Ärztin in Deutschland nicht mehr werden zu können oder zu wollen. Ein neues Vorbild, an dem sie sich an Stelle dessen orientieren könnte, hatte sie im Kontext des Studiums bisher nicht gefunden, obwohl sie von einigen positiven Erlebnissen mit Ärzten erzählte (S14 103).

Negative Erfahrungen, die eine Diskrepanz zwischen Ideal und real erlebtem Verhalten darstellten, konnten zur Verwirrung und Unsicherheit der Studierenden beitragen. Zum Eindruck, das Praktizieren von Werten sei angesichts von Arbeitsbelastungen im Beruf nicht möglich, trugen teilweise Erfahrungen im Studium bei (vgl. u.a. S14 083). Dies bezog sich auch auf das Ideal einer guten medizinischen Ausbildung. S05 berichtete z.B. von einem Praktikum, bei dem zur gleichen Zeit zwölf Famulanten von Ärzten betreut werden mussten (S05 071).

Auch Personen, die gerade nicht als „Vorbild“ gesehen wurden, spielten eine zentrale Rolle bei der Auseinandersetzung mit ärztlichen Idealen. Erfahrungen mit innen 
Ergebnisse

wurden von den Studierenden als prägend beschrieben (vgl. S08 10). Sie waren Personen, mit denen sie negative Erfahrungen gemacht hatten, ,Anti-Vorbilder': ein Inbild dafür, welche Ärzte sie nicht werden wollten (vgl. S14 09, S12 48). Negative Erfahrungen und damit verbundene Gefühle wie „Frust“ trugen dazu bei, „zu lernen“ und an den Situationen „zu wachsen“ oder „stärker zu werden“ (vgl. S08 10, S05 011).

Eine Orientierung erfolgte somit nicht nur an positiven Vorbildern, sondern auch an Negativbeispielen. Gerade die Diskrepanz zwischen Idealvorstellungen und Realität könnte einen Lernprozess bei den Studierenden provozieren. Ernüchternde oder negative Ereignisse wirkten jedoch gleichermaßen demotivierend auf die Studierenden. Als wichtiges Ergebnis zur Vorbildwirkung lässt sich jedoch festhalten, dass das beobachtete Verhalten der Ärzte und anderer Interaktionspartner nicht unmittelbar übernommen oder gar eins zu eins imitiert, sondern von den Studierenden bewertet, hinterfragt und teilweise abgelehnt wurde. Abbildung 6 fasst die Ergebnisse zum Thema „Auseinandersetzung mit Idealen der ärztlichen Profession“ im Sinne des Kodier-Paradigmas zusammen: 


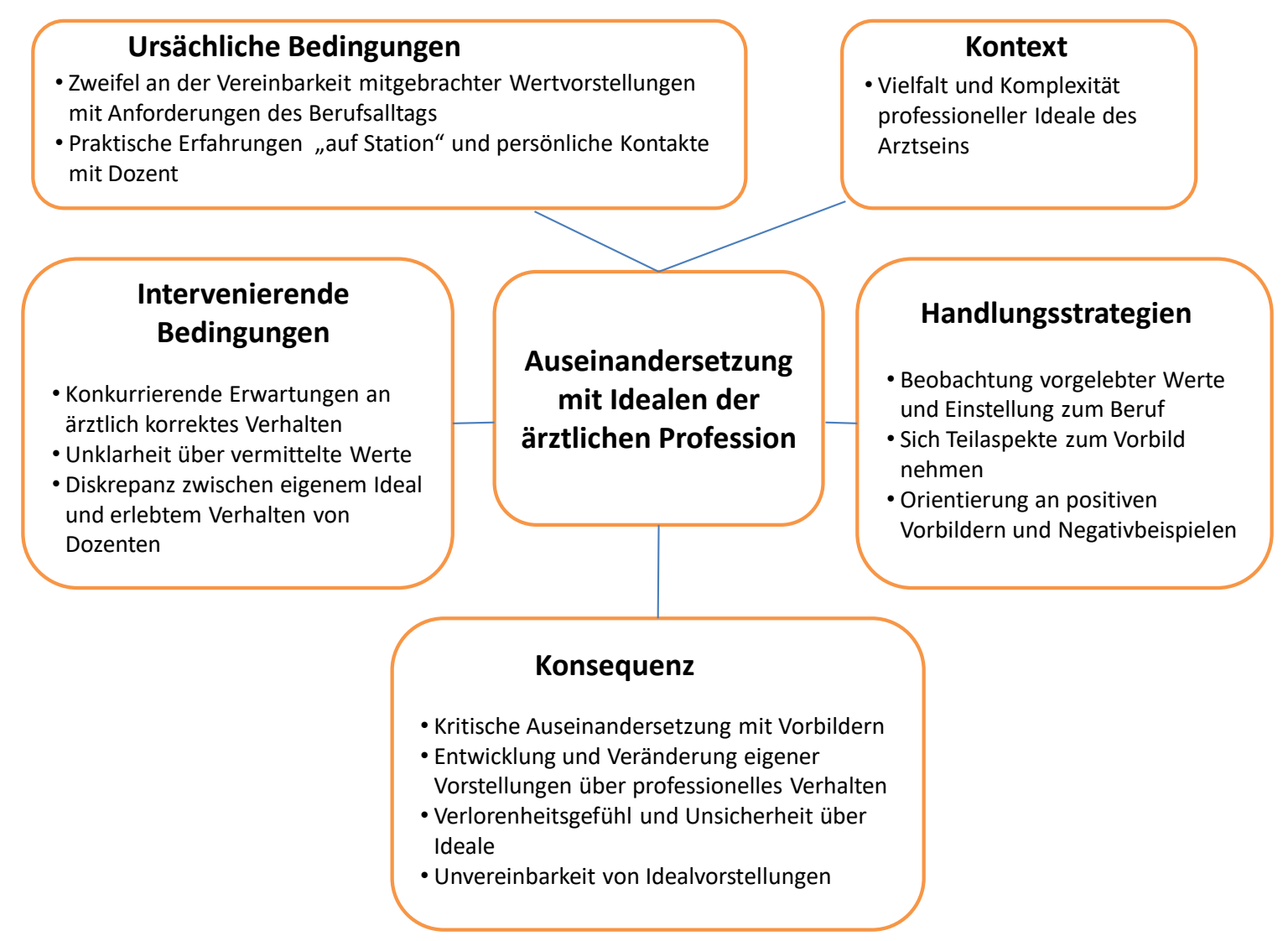

Abbildung 6: Kodier-Paradigma zum Thema „Auseinandersetzung mit Idealen der ärztlichen Profession“.

\subsubsection{Rolleneinübung: Auseinandersetzung mit der Rolle als Medizinstu- dent/in und Antizipation der Arztrolle}

\section{Das Phänomen}

Die Studierenden beschrieben, wie sie sich im Studium mit der angestrebten Arztrolle auseinandersetzten und nach Möglichkeiten suchten, in die Rolle hineinzuwachsen und sie zukünftig auszufüllen. Durch den Kontakt zu Ärzten würden sie besser einschätzen können, was der Arztberuf im Einzelnen beinhaltet und zugleich die Arztrolle auch aus einer Beobachter-Position heraus antizipieren können. Teilweise schien die dabei erfolgende Vorbereitung auf den Beruf unbewusst und von Erfahrungen mit anderen Mitgliedern der Fakultät geprägt zu sein. Wie bereits früher angesprochen, orientierten sich die Studierenden in Bezug auf Einstellungen zum Studium wie z.B. in ihrer Arbeitseinstellung stark an ihren Kommilitonen. Diese vermittelten als erste und häufigste Kontakte zu Beginn des Studiums, was relevant und zur 
Ergebnisse

Bewältigung der Anforderungen erforderlich war. Deren Sichtweise des Berufes wiederum wurde vor allem durch Ärzte in Praktika beeinflusst. Die Studierenden schienen in ihnen eine ,Vorschau' der zukünftig zu erfüllenden Rollen im Sinne einer Orientierung zu sehen (vgl. Kapitel 5.2.1: Orientierungshilfen durch Vorbilder: Die Rolle der Kommilitonen).

\section{Kontext}

Bei Studienbeginn war es für die Studienteilnehmer zentral, in die Rolle von Medizinstudierenden hineinzufinden. Dabei wirkten Haltungen und Erfahrungen von anderen Mitgliedern der Fakultät auf sie ein (vgl. auch S05 003, S13 003):

„der X-(Name eines Kommilitonen) hat natürlich ne sehr wie soll ich sagen also vielleicht so ne spezielle Einstellung zu den Dingen und das hat mir hat mich glaub ich geprägt darin //l:mhm// es war eine sehr menschliche Einstellung sehr .. sozial auch“" (S08 03).

Vorherrschende Vorstellungen über „gute Studenten“ wie Leistungsstärke und Durchhaltevermögen beim Lernen wurden ebenfalls von älteren Studierenden vermittelt (vgl. S03 27). Ängste, Anforderungen im Studium nicht erfüllen zu können, wurden dabei manchmal verstärkt:

„einfach so viel Anspannung auf //l: $\mathrm{mhm} / /$ ähm... ja wegen nix und wieder nix aber man steigert sich dann da so rein“ (S03 27).

Auch unter Medizinstudenten vorherrschende Umgangsformen beeinflussten Verhaltensweisen des Einzelnen:

„also ich find das immer schwer auch gut mit anderen Leute zu lernen weil ja das ja auch schon ein Studium ist das sehr viele Leute mit sich bringt die eben nicht zugeben können wenn sie mal was falsch machen oder //l:mhm// mal zugeben können und immer so dieses anstrengende auf seiner Meinung beharren" (S13 076).

Die Studierenden machten sowohl die Erfahrung, dass ältere Studierende innen in Bezug auf die Bewältigung des Studiums durch ihr Verhalten „die Angst nahmen“ (S07 08) als auch, dass sie diese verstärken konnten. Von vorherrschenden Rollenbildern abweichendes Verhalten konnte dabei das Bild der angestrebten Rolle verändern (vgl. S15 027 und 031).

Vorbilder vermittelten in diesem Zusammenhang die im Studium vorherrschende „Medizinkultur“ und erwünschte Verhaltensweisen und Einstellungen im Sinne akzeptierter medizinischer Praxis. Die Rolleneinübung als Arzt/Ärztin ist somit nicht nur eine Auseinandersetzung mit persönlichen, sondern auch mit gemeinsamen Idealen 
und Erfahrungsinhalten einer Fakultät. Das Medizinstudium wirkte dabei wie ein Kulturraum, innerhalb dessen bestimmte Einstellungen und Verhaltensweisen praktiziert, erhalten und weitergegeben werden ${ }^{23}$. S08 erwähnte z.B. einen Dozenten als prägend, den er als "Genie" beschrieb und der inn aus dem Grund „beindruckte“, weil er "viel wusste" (S08 05). Auch ohne direkten Kontakt wurde hier durch die Person des Dozenten ein Maßstab für eine „erwünschte Existenzweise“ eines Arztes gesetzt.

\title{
Ursächliche Bedingungen
}

Die Notwendigkeit, sich auf die professionelle Rolle vorzubereiten, wurde den Studierenden vor dem Hintergrund des bevorstehenden Berufseintritts bewusst. Sie gaben an, durch Vorbilder für den Beruf relevante Inhalte gelernt zu haben. S08 z.B. lernte von einem Arzt, sich auf einer "kommunikativen Ebene [...] geschickt" zu verhalten (S08 09). S05 fiel auf, dass ihr eigenes Auftreten sich erheblich von dem professionellen Verhalten einer Ärztin unterschied und dies Relevanz für Patienten hatte. Auch andere Studierende hatten durch die Beobachtung von Interaktionen zwischen Ärzten und Patienten wichtige Erkenntnisse über den Patientenumgang gewonnen (vgl. auch S04 079, S13 007):

\begin{abstract}
„aber dass sie medizinisch Sicherheit gegeben hat und ähm.. emotional ähm //l:mhm// auch damit umgehen konnte wenn Emotionen gezeigt wurden äh sich dadurch nicht hat verunsichern lassen also //l:ja// ist halt bei also ich lass mich dann immer verunsichern wenn //l:ja// vor mir ein Patient weint" (S05 047).

„das zum Beispiel ähm es ein Riesenunterschied für den Patienten macht wie man.. ob man unsicher als Arzt auftritt oder ob man den Patienten Sicherheit vermitteln kann" (S05 003).

„,ich fand die Art ähm wie sie mit Patienten umgehen also die Art wie sie Gespräche führen fand ich ähm //l:mhm// .. fand ich irgendwie vielleicht hab ich es vorher noch sie so bemerkt //I:ja// aber sie hatten eben diese Art nem Patienten zuzuhören //I:mhm// und ausreden zu lassen auch mal zuzulassen dass der Patient noch überlegt [...] und somit vielleicht sogar auch weiterkommt" (S05 127).
\end{abstract}

${ }^{23}$ Der Begriff ,Medizinkultur‘ wurde bereits im Kapitel 2: „Stand der Forschung“ definiert. Speziell sind hier auf Studierende einflussnehmende kulturelle Umstände gemeint, denen sie allein dadurch ausgesetzt sind, dass sie sich im Raum der medizinischen Fakultät und damit verbundenen Orten wie Lehrkrankenhäusern bewegen. Dieser Raum wird im Folgenden als „Kulturraum Medizin“ bezeichnet vgl. Fußnote 3 und 24. 
Ergebnisse

Die unterstützende Betreuung durch Lehrende und ihr positives Feedback z.B. in Bezug auf den Kenntnisstand (S03 61, S07 035) erleichterten die Rolleneinübung. Die neue Rolle in Situationen auszuüben, die noch ungewohnt waren, empfanden die Studierenden als herausfordernd (vgl. S13 032). Ihre Eindrücke vom „medizinischen Umfeld“ waren entscheidend dafür, ob sie sich dort wohl und sicher fühlten:

„der ähm hat immer gesagt ja ist nicht so schlimm und ist klar ihr fangt erst an und . ähm .. ich
hab das mitbekommen dass zu jemanden anderen der das erste Mal genäht hat der gezittert
hat wie ähm noch was das ist auch normal //l:mhm// ähm hier jetzt komm ist kein Stress und
das machste nochmal dann wird's noch besser und ist aber schon gut und vielen Dank dass
du mir halt geholfen hast und //l:mhm// es war irgendwie klar dass du ihm jetzt nicht so viel
geholfen hast aber der hat das halt mit so einer so einer Überzeugung gesagt dass //l:ja// man
dachte okay vielleicht hab ich doch irgendwas geholfen und ähm ja dieses Gefühl eben dass
er sagt . okay das war jetzt nicht nicht ganz umsonst [...]von dem zusätzlichen Gefühl dass
man eben dass das selber sinnvoll war //l:mhm// ja okay war klasse dass ihr da wart“ (S13
064).

Der Arzt vermittelte den Studierenden das Gefühl, dass sie willkommen sind, dass sie als „Lernende“ noch nicht alles können müssen (vgl. auch S13 003) und dass sie eine Hilfe sind. Davon, in den klinischen Alltag einbezogen und unterstützt zu werden, berichteten jedoch nur wenige.

Für eine erfolgreiche Rolleneinübung waren auch die seitens der Studierenden wahrgenommenen Erwartungen ihres Umfelds von Bedeutung:

„am Anfang [hatte ich] das Gefühl ich muss da jetzt erst mal mich beweisen //l:mhm// und irgendwie zeigen dass ich mich jetzt nicht für was Besseres halte nur weil ich eben Medizin studiere" (S06 43).

Dass die Studentin versuchte, ihr eigenes Verhalten an die vermuteten Erwartungen des medizinischen Umfelds anzupassen, zeigt den Einfluss der bestehenden Medizinkultur. Weniger Interesse oder Lerneifer glaubt sie unter Beweis stellen zu müssen, um akzeptiert zu werden, sondern die Bereitschaft, sich unterzuordnen. Dazu passt auch der häufig geäußerte Eindruck der Studierenden, im Praktikum dem Personal im Wege zu stehen:

„wenn man dann nur im Weg steht und nur nervt und auch ähm was heißt nervt aber ist ja schon anstrengend ist für alle Beteiligten und die Leute da auch nicht so richtig Bock haben wenn's da abgeht“" (S13 052)

Der Eindruck, eine Belastung für die Angestellten zu sein, beeinflusste und veranlasste S13 zu dem Wunsch, sie durch seine Anwesenheit nicht „zu nerven“ (S13 
064). Von der Erfahrung, Vorstellungen der medizinischen Fakultät über "gute Studierende" nicht zu erfüllten, erzählte vor allem Studentin S12. Sie selbst schien diese Vorstellungen übernommen zu haben, da sie sich als "schlechtes Beispiel“ einer Medizinstudentin bezeichnete (S12 04). Sie sei „anders“ als andere Medizinstudenten und berichtete von Insuffizienzgefühlen bezüglich ihrer Leistungen im Studium (vgl. S12 04 und 44), weshalb das Studium ihr wenig Freude bereitete und sie sich „rauszog" (S12 04 und 17).

Dem gegenüber stehen Erfahrungen anderer Studierender, deren Beziehung zu anderen Mitgliedern der Fakultät durch "Nähe“ gekennzeichnet war. Voraussetzung dazu war eine Bereitschaft der Dozenten, mit Studierenden in Kontakt zu treten. S04 beschrieb einen Arzt als "nahbar", dem es wichtig zu sein schien, welchen Eindruck Studierende von ihm erhielten (vgl. S04 75). Er zeigte sich ihnen gegenüber „offen“, ohne "Statusgehabe", um respektiert zu werden (vgl. S04 007 und 073). Vielmehr überzeugte er durch sein zugewandtes und bedachtes Verhalten. Ein enger Kontakt zu Vorbildern, die höhere Positionen bekleideten, stellte sich nur in Ausnahmefällen ein.

Ob sich Studierende dazu entschlossen, sich in ihrer Rolleneinübung an Ärzten zu orientieren, hing gleichsam davon ab, ob diese die von innen angestrebten Ziele weitgehend verkörperten (vgl. S04 003). Umgekehrt mussten die Studierenden auch bereit sein, ihr eigenes Verhalten anzupassen. Einige schienen ein Problem damit zu haben, die Arztrolle einzuüben, wenn von innen gefordert wurde, sich aufzuopfern oder die Bereitschaft zu zeigen, auch extreme Zustände auszuhalten, um Leistungen zu erbringen. In der Prüfungsvorbereitung großen Stress aushalten zu müssen, wurde als Normalität geschildert. Der Stress würde noch dadurch verstärkt, dass andere Kommilitonen Maßstäbe für ein hohes Lernpensum setzten (vgl. S11 031).

\section{Intervenierende Bedingungen}

Den Studierenden war die Orientierung an Ärzten wichtig für die Rolleneinübung. Umso besorgter waren sie über den Mangel an Kontakten mit ihren Dozenten und wünschten sich häufigere Rückmeldungen (vgl. S03 31, S07 04 und Kapitel 5.2.4: Auseinandersetzung mit Idealen der ärztlichen Profession). Sie berichteten, ihre Dozenten oft nicht als Personen zu kennen (S01 04). S07 wirkte deshalb unsicher, ob ein Professor ein Vorbild für inn sein könne: 
Ergebnisse

„I: Würdest du so jemand jetzt auch als Vorbild bezeichnen? P:Ja schon //l:mhm// also vor als Vorbild äh als Professor ne ich weiß nicht wie der als Mensch ist“" (S07 051).

Die Studierenden fühlten sich von Ärzten ausgeschlossen, wenn sie z.B. während eines Praktikums kein Interesse an innen zeigten (vgl. S06 03) oder innen das Gefühl gaben, erst für besondere Leistung akzeptiert und gefördert zu werden. Beim Lernen und in Bezug auf erste klinische Erfahrungen hatten Studierende den Eindruck, auf sich allein gestellt zu sein und äußerten, sich „allein gelassen“ zu fühlen (vgl. S13 032, S07 04).

Angesichts des Eindrucks, das Studium sei „nur auf Leistung“ ausgerichtet, und eines Mangels an Vorbildern fürchteten die Studierenden einen negativen Einfluss auf inr zukünftiges Verhalten als Ärzte (S03 47). S03 sagte, einige Kommilitonen „vergessen, dass sie „nen sozialen Beruf lernen“ (S03 47). Explizit äußerte S07 den Wunsch, im Studium durch Dozenten auf die soziale Komponente des Berufes vorbereitet zu werden (S07 10), statt „alles auch aus den Büchern zu lernen“ (vgl. S07 04). Auch andere Studierende wünschten sich eine persönlichere Lehre im medizinischen Kontext, da dies das Lernen erleichtere (S14 55).

Eine fehlende Betreuung durch Lehrende bei ersten Patientenkontakten und praktischen Tätigkeiten erschwerte die Rolleneinübung und führte zu Unzufriedenheit und großer Frustration. S08 berichtete von seinen Erfahrungen während des Praktischen Jahres. Für S15 minderte ein Mangel an praktischen Erfahrungen ihre Motivation im Studium.

„und das ist was man im PJ generell mal sagen müsste dass das völlig fehlt //l:mhm// dass Leute irgendwie Interesse haben Lehre zu machen //I:mhm// dieser blöde Satz von wegen Eigenverantwortung kannste völlig vergessen //l:mhm// wenn die Leute keine Lust darauf haben da kannste dich sonst so viel reinhängen dass wird nie klappen wenn das nicht von beiden Seiten aus kommt" (S08 068).

„weil ich das Gefühl hab man sitzt in der Uni und lernt so viel und macht so viel theoretisch //l:ja// und sitzt in den Hörsälen ohne Fenstern mit der Klimaanlage und das deprimiert viel und ähm .. deshalb gab manchmal schon so Situationen ähm ... wo ich dann mehr den Sinn gesehen hab auch“ (S15 015).

Ähnlich überfordernd wirkten Ereignisse, bei denen Studierende keine Unterstützung erhielten:

„und irgendwie haben die also die Schwestern haben das mit Sicherheit nicht so gemeint aber die haben schon auch gesagt ja du warst nicht da und äh jetzt ging's inm schlecht und so 
Ergebnisse

//l:mhm// und ich hatte auch Angst dass ich inn vielleicht angesteckt hab dadurch dass ich inn so rumgetragen hab dass ich war son bisschen erkältet in der Woche davor und ich dachte auch vielleicht //l:mhm// hab ich inn auch irgendwie mit irgendwas angesteckt und er hat das dann nicht gepackt oder so also es war irgendwie .. ganz schlimm für mich so das also ja ich hatte da nicht mit gerechnet und hatte auch das Gefühl dass ich da vielleicht sogar noch was dazu beigetragen hab“" (S06 55).

Da S12 sich weder mit dem Studium noch mit der Arztrolle identifizieren konnte (vgl. Kapitel 5.2.3: Identifikation mit Ärzten, Studium und Beruf), zweifelte sie ihre Rolle als Medizinstudentin an und sagte über ihr eigenes Verhalten im Studium: „Das könnte man sicher auch anders handhaben“ (S12 04). Ebenso wenig erfüllte S05 in ihrem Erleben die Erwartungen an eine „gute Studentin“, als sie durch eine Prüfung fällt. Beide Studentinnen suchten Hilfe bei anderen Mitgliedern der Fakultät. Ein Unterschied bestand jedoch in ihrer Beziehung zu den gewählten Personen: S05 scheint die Professorin, der sie sich anvertraut, als Vorbild zu erachten; S12 erkennt zwar an, dass ihre Kommilitonen, die sie beim Lernen unterstützten, ihr „wirklich weitergeholfen“ haben (S12 04), grenzt sich jedoch von innen ab. Sie gab an, sie nicht „sympathisch" zu finden und keine Gemeinsamkeiten mit innen zu haben (vgl. S12 44). Der Unterschied scheint darin zu liegen, dass S12 sich im Gegensatz zu S05 nicht mit den helfenden Personen und auch nicht mit dem Studium identifiziert (vgl. S12 15 und 46, S05 095):

„ja und ich bin halt auch irgendwie in diesem Medizinstudium gelandet und steh da aber auch nicht ganz hinter das ist //I:mhm// .. ja das motiviert dann natürlich nicht so ne" (S12 46).

\section{Handlungsstrategien}

Sich in der ärztlichen Rolle „auszuprobieren“, geschah zum einen durch die Übernahme ärztlicher Aufgaben in Praktika. Wenn Ärzte Vertrauen in die Arbeit von Studierenden hatten, wirkte sich das positiv auf deren Selbstvertrauen aus:

„in der Chirurgiefamulatur das war meine zweite Famulatur und da haben die mich dann alleine die Aufnahmen machen lassen [...] wenn das halt nicht geht dann ist das viel eindrücklicher ähm als wenn oder hebt es nochmal hervor wie gut des ist wenn man einem was zutraut" (S11 111).

Zum anderen übten sich Studierende in die zukünftige Rolle durch Beobachtung und Beurteilung des Verhaltens von Ärzten und Kommilitonen ein. Dabei beurteilten sie das Verhalten der Ärzte in Bezug auf deren Vorbildfunktion und verglichen es mit 
Ergebnisse

eigenen Verhaltensmaßstäben (vgl. u.a. S05 047, S04 079): ob sie es für angemessen hielten und wie sie sich selbst als Ärzte verhalten würden (S11 83).

Die Beobachtung prägender Personen bot Identifikationsmuster für die künftige professionelle Tätigkeit. Auf den ersten Blick wirkt es skurril, wenn S04 einen Freund beschreibt, der sich „wie ein Chirurg zu rasieren“ versucht (S04 031). Deutlich wird, wie auch die Wahrnehmung der Lehrenden als Privatpersonen das Bild von Ärzten der Studierenden beeinflusst und das Professionelle in das Private reicht bzw. erst dadurch zur Orientierung wird.

In Bezug auf die Rolle als Studierende orientierten sie sich an ihren Kommilitonen, auch wenn sie teilweise der Meinung waren, ihre Kommilitonen würden sich zu sehr von den Anforderungen im Studium unter Druck setzen lassen (S04 051, S11 031). Auf der anderen Seite schilderten die Studierenden das Erfordernis, sich gegenseitig darin bestärken zu müssen, sich nicht von dem Stress anderer anstecken zu lassen und im Studium selbstfürsorglich mit sich umzugehen (vgl. auch S08 04 und 32, S03 43):

„,ich glaub das war auch ganz gut dass äh.. wir haben uns dann glaub ich auch gegenseitig auch manchmal dann motiviert noch zum Sport zu gehen weil dann denkt man so oh ne ich hab gar keine Zeit [...] ich hab noch so viel zu tun und so und dann hieß es [...] komm jetzt lass uns gehen ähm das tut uns gut und da gehen wir jetzt hin“ (S03 27).

Selten sprachen Studierende mit Dozenten über Probleme bei der Bewältigung ihrer Rolle. Wie bereits geschildert, vertraute sich S05 mit einer nicht bestandenen Prüfung einer Dozentin an und nahm dabei das Risiko in Kauf, als „schlechte" Studentin beschämt zu werden (vgl. S05 095). Die Professorin begegnete ihr jedoch mit Unterstützung und bestätigte die Studentin dadurch in ihrem Verhalten.

\section{Konsequenzen}

Wenig Betreuung während erster klinischer Praktika und ein ablehnendes Verhalten seitens des medizinischen Personals erschwerte die „Erprobung“ der neuen Rolle. Demotivierend war es, wenn Lehrende Statusunterschiede oder Überlegenheit betonten und sich Studierende dadurch gedemütigt und untergeordnet fühlten (vgl. S04 007 und 091). S04 erzählte von einer Vorlesung, in der ein Dozent öffentlich die Unterlegenheit einer Studentin demonstrierte, indem er ihr Wissen über eine Krankheit als mangelnd rügte und ihr das Gefühl gab, den Erwartungen an Medizinstudenten nicht gerecht zu werden (vgl. S04 051). Das Gefühl, als Student/in nicht akzeptiert 
Ergebnisse

oder gar abgelehnt zu werden, hatte Auswirkungen auf die Identifikation der Studierenden mit ihrer Rolle als Medizinstudierende (vgl. Kapitel 5.2.3: Identifikation mit Ärzten, Studium und Beruf). Es schien ihnen wichtig zu sein, von ihren Dozenten in ihrer Rolle als „gute Studenten“ bestätigt zu werden:

„,ich glaub bei mir war das in der Schule schon so [...] dass es mir auch wichtig war dass die Lehrer und Lehrerinnen auch nen guten Eindruck so von mir haben“ (S03 31).

Ein Bedürfnis nach Lob und Anerkennung durch Dozenten konfligierte mit der Erfahrung, von innen ignoriert oder abgewertet zu werden (vgl. S03 61, S06 03), und führte zu einem Konflikt mit der eigenen Rolle. Ärzte, die ein Rollenbild vermittelten, das nicht mit den persönlichen Erwartungen und Bedürfnissen der Studierenden zu vereinen war, wurden zwar abgelehnt, hatten aber trotzdem Einfluss.

Zu einem Konflikt führte nicht nur die Befürchtung, Erwartungen der Lehrenden, sondern auch eigene Erwartungen nicht erfüllen zu können. So litt S06 unter dem Eindruck, etwas falsch gemacht zu haben und den damit verbundenen Schuldgefühlen (vgl. S06 55). Die Rolle der Studierenden und damit verbundene Anforderungen schienen in vielen Lehrsituationen nicht geklärt zu sein.

Zu den größten Verunsicherungen führten Erfahrungen im Krankenhaus, bei denen Studierende durch Nichtbeachtung oder Missfallen über ihre Anwesenheit den Eindruck gewannen, ihre Anwesenheit sei unwichtig und störend (S13 052) und dies Minderwertigkeitsgefühle nach sich zog (vgl. S12 04 und 42, S07 55).

Wenn dagegen Ärzte die Studierenden darin bestärkten, die Möglichkeit des Lernens im Studium zu nutzen, machte dies Studierende wie S13 zuversichtlicher und selbstbewusster in Bezug auf die eigene Rolle und die Zukunft als Arzt/Ärztin.

„aber der hat immer gesagt wenn irgendjemand sagt zu euch ähm ihr sollt gehen oder euch nicht antwortet auf eure Fragen dann ist das halt ein Idiot und das ist gut dass ihr hier seid und so //I:ja// ist doch nett dass ihr uns helft ((lacht)) und das find ich echt cool“ (S13 52).

Dozenten und Ärzte, die bereits länger im „Kulturraum Krankenhaus/medizinische Fakultät" agierten, gaben den Studierenden Verhaltens-Standards und setzten Maßstäbe sozialen Verhaltens. Mehrere Studierende erlebten es als positiv, von ihren Tutoren beim Lernen "gepusht" zu werden und sahen sich dadurch motiviert, diszipliniert zu arbeiten (vgl. S12 17, S07 43). Andere fühlten sich unter Druck gesetzt und kritisierten, dass Tutoren ihre Situation damit verschlechterten (S15 027), da eine "Angst besetzte Atmosphäre" dem Lernen nicht förderlich sei. Vielmehr wünschten 
Ergebnisse

sich die Studierenden eine Lernumgebung, in der Fehler gemacht und offen damit umgegangen werden dürfte. Die Bereitschaft, Forderungen von Lehrenden zu akzeptieren, war größer, wenn diese die Studierenden bei ihrem Wissenserwerb unterstützten und sie als angehende Ärzte willkommen hießen. Eine gelungene Identifikation mit prägenden Personen im Studium erleichterte die Rolleneinübung des Arztberufes und die Identifikation mit der Arztrolle (vgl. Kapitel 5.2.3: Identifikation mit Ärzten, Studium und Beruf). Dabei half auch der Kontakt zu Ärzten, die selbst ihren individuellen Weg im Beruf gesucht hatten (vgl. auch S11 09):

„hab einfach gemerkt wow irgendwie gibt's auch Menschen die ham ähnliche Interessen und die finden das auch alles scheiße //l:mhm// und du wirst aber deine Nische finden" (S12 39).

Wenn Dozenten die Bereitschaft zeigten, auf einer persönlichen, nicht-hierarchischen Ebene mit Studierenden in einen direkten Kontakt zu treten, konnten sie am eigenen Beispiel ärztliche Eigenschaften wie Empathie-Fähigkeit vermitteln. Autorität und die Wahrnehmung als Vorbild beruhten hier gerade auf Vertrauen, fehlender Angst und einer als gleichberechtigt empfundenen Beziehung. Ärzte, die die Studierenden unterstützten und ihr Selbstbewusstsein stärkten, halfen innen auch dabei, sicherer in ihrer Rolle als Studenten zu agieren (vgl. S05 011, S13 052, S01 35):

„,ich weiß nicht die die haben das so über Trick 17 gemacht //(lacht))// man hatte nie das Gefühl man ist blöd //l:ja// aber sie haben einem gleichzeitig noch in diesem Seminar so viel Wissen mitgegeben aber man hatte auf der anderen Seite das Gefühl man hat ja auch selber schon ein bisschen Wissen“ (S05 063).

Zusammenfassend zeigte sich die Rolleneinübung als fortlaufender Prozess von Anpassung, Distanzierung und Modifizierung eigenen Verhaltens in Bezug auf eine bestehende ,Medizinkultur' und in Interaktion mit ihren Mitgliedern. Sie beinhaltete eine Reflexion der aktuellen Rolle als Studierende und der zukünftigen Rolle als Ärzte. Abbildung 7 fasst die Ergebnisse zum Thema „Rolleneinübung: Auseinandersetzung mit der Rolle als Medizinstudent/in und Antizipation der Arztrolle" im Sinne des Kodier-Paradigmas zusammen: 


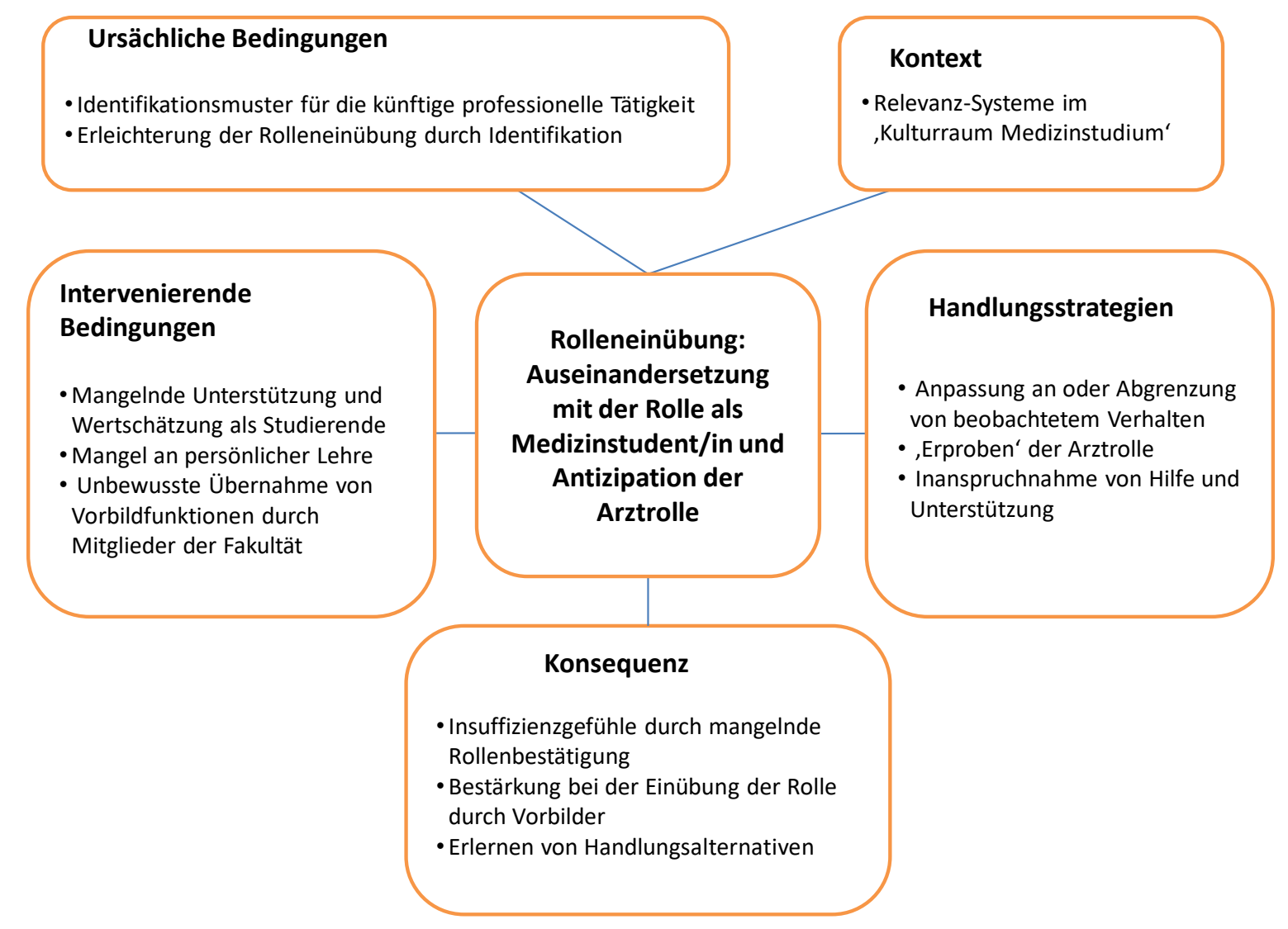

Abbildung 7: Kodier-Paradigma zum Thema „Rolleneinübung: Auseinandersetzung mit der Rolle als Medizinstudent/in und Antizipation der Arztrolle“.

\subsubsection{Entwicklung von Strategien zur Bewältigung des Studiums}

\section{Das Phänomen}

Die Studierenden berichteten, im medizinischen Umfeld ,das Charakteristische' des Kulturraums Medizin v.a. durch den Kontakt zu klinisch tätigen Ärzten zu lernen. Dabei waren sie nicht passiv, sondern entwickelten Strategien, um sich in der Rolle als Medizinstudierende und dem medizinischen Umfeld zurechtzufinden. Die (teils unbewusste und zufällige) Kontaktaufnahme mit Vorbildern war dabei ein wichtiges Element.

\section{Kontext}

Das Medizinstudium, das u.a. aufgrund festgelegter Lehrpläne als ,verschult' gilt, zeichnet sich durch strikte explizite Anforderungen und Handlungsanweisungen aus. 
Ergebnisse

Zugleich vermittelt es wie kaum ein anderes Studium umfangreiches implizites Wissen auf informellem Weg, einschließlich des Erlernens „professionellen ärztlichen Handelns“. Im „Kulturraum Medizin“ prägen vorherrschende gemeinsame Ideale und unausgesprochene Verhaltensregeln die Situation der Studierenden von Beginn an. Studierende sprachen häufig über Druck, Anforderungen genügen und ihr Können unter Beweis stellen zu müssen, und über die Unklarheit bezüglich ihrer eigenen Rolle als Medizinstudierende (vgl. Kapitel 5.2.4: Auseinandersetzung mit Idealen der ärztlichen Profession).

\section{Ursächliche Bedingungen}

Wenn sich Studierende bei der Erschließung professioneller Rollen von Seiten der Fakultät wenig unterstützt fühlten, entwickelten sie alternative Handlungsstrategien oft aus dem Wunsch nach Zugehörigkeit und Akzeptanz. Positive direkte Erfahrungen mit Vorbildern erleichterte es den Studierenden, Strategien zur Bewältigung des Studiums und zur Einübung der Arztrolle zu entwickeln. Zwischen Kommilitonen wurden Bewältigungsstrategien, Interessensschwerpunkte und Haltungen zum Studium ausgetauscht. Dabei hatten Kommilitonen ähnliche Vorbild-Funktionen wie Ärzte (vgl. Kapitel 5.2.3: Identifikation mit Ärzten, Studium und Beruf).

Selten erfolgte die Entwicklung von Strategien in einem direkten ,Erfahrungsaustausch' mit Lehrärzten. Nur S12 berichtete, sich mit einer Dozentin über ein persönliches Problem, in ihrem Fall Prüfungsangst, ausgetauscht zu haben. Diese Strategie, bei der Erfahrungen geteilt werden und man wechselseitig voneinander profitiert, berichteten die Studierenden in Bezug auf Kommilitonen recht häufig. Dabei ging es nicht nur um medizinische Inhalte der Fakultät im engeren Sinn, sondern auch z.B. um Fragen zur sich an das Studium anschließenden Spezialisierung.

„ja wenn wir wenn ich da war oder wenn er da war quatschen wir halt viel darüber und ähm ja ist einfach en nen Austausch auch nicht über das war wir gerade machen auch das was wir mal machen wollen und //l:mhm// des ist einfach ich weiß nicht ich find das cool wenn man so .. wenn man sich da so austauschen kann der hat auch schon was gesehen //l:mhm// also das achte ich auch selber weil ich viel auch mitkriege der macht viel auch sonst so Praktika oder so.. und dann erzählt er immer was da abgeht und dann ähm .. kann man sich da zusammen son Allgemeinwissen da erarbeiten“" (S13 076).

„also nicht nur so Facts sondern auch so wie gesagt so bisschen ja was was später mal draus wird oder was wir machen wollen welche Richtung es geht //l:mhm// ja das ist schon wichtig“" (S13 080). 
Ergebnisse

Hinsichtlich der beruflichen Orientierung wurden Ärzte auf Station genau beobachtet. Anders als mit Kommilitonen war mit Dozenten weniger das Gespräch von Bedeutung. Vielmehr diente ihre Beobachtung den Studierenden zum Vergleich und der Frage, ob sie sich mit dem ärztlichen Verhalten und Auftreten identifizieren konnten (vgl. Kapitel 5.2.3: Identifikation mit Ärzten, Studium und Beruf). Der Vergleich war gewissermaßen eine Selbstreflexion, die durch die Interviewsituation explizit als Strategie erkannt wurde:

„aber dass sie medizinisch Sicherheit gegeben hat und ähm.. emotional ähm //l:mhm// auch damit umgehen konnte wenn Emotionen gezeigt wurden äh sich dadurch nicht hat verunsichern lassen also //l:ja// ist halt bei also ich lass mich dann immer verunsichern wenn //l:ja// vor mir ein Patient weint //l:mhm// und die irgendwie damit ziemlich gut umgehen konnten" (S05 047).

Dozenten, die die Studierenden auf Station einbezogen und sie an Interaktionen mit Patienten beteiligten, erleichterten die innere Vorbereitung auf die Arztrolle. Direkte positive Erfahrungen mit Vorbildern ermöglichten es ihnen erst, ärztliches Verhalten als „richtig“ zu erleben und zu der Überzeugung zu kommen, es sich aneignen zu wollen. Überzeugend wirkte besonders ein Verhalten von Ärzten, bei dem die Studierenden selbst so behandelt wurden, wie es als richtiges Verhalten Patienten gegenüber gelehrt wurde.

Eine Vorbildfunktion bei der Rolleneinübung übernahmen auch Personen, zu denen keine besondere Beziehung bestand und die die Studierenden nicht als ,Idealbild“ definierten. Wenn es um die Demonstration eines in einem Kulturraum geläufigen und erwünschten Verhaltens geht, können sich Studierende an negativen Vorbildern genauso orientieren wie an positiven, und gerade nicht als unreflektierte Übernahme von Eigenschaften. Strategien für eine Reflexion von beobachtetem Verhalten erfordern vielmehr aktive Entscheidungsprozesse von Seiten der Studierenden.

Auffällig war, dass trotz einer Bewusstheit für ,erwünschtes Verhalten im Kulturraum Medizin' offensichtlich viele Studierende einen Wunsch nach einem von der Norm abweichendem Verhalten hatten. Häufig wurde gerade Verhalten, das als Ausnahme wahrgenommen wurde, bewundert und gelobt. Einige der Studierenden erachteten es z.B. als vorbildlich und zugleich außergewöhnlich, mit Fehlern und „Schwächen“ offen umzugehen (vgl. S13 076). Die Diskrepanz zwischen eigenem Ideal und real Erlebtem verhinderte eine ausschließliche Orientierung an Mitgliedern der Fakultät 
Ergebnisse

und motivierte zur Entwicklung eigener Maßstäbe und die Umsetzung eigener Strategien.

\section{Intervenierende Bedingungen}

Ärzte, auch wenn sie allgemeinen Verhaltensmaßstäben gemäß agierten, waren nicht unbedingt Vorbilder; sie mussten zumindest teilweise den Verhaltensmaßstäben und -idealen der Studierenden entsprechen. Kritisiert wurde beispielsweise hierarchisches Verhalten, gerade weil sich Studierende in der Wissensvermittlung von ihren Dozenten abhängig fühlten. Sie orientierten sich besonders auf Station an dem Verhalten des Personals, um Hinweise zu erhalten, was von innen verlangt wurde. Sich unausgesprochene Regeln selbstständig, allein durch aufmerksame Beobachtung und ohne die Unterstützung derer, denen sie sich untergeordnet fühlten, aneignen zu müssen, war aber nur begrenzt möglich und oft belastend.

Die Entwicklung von Bewältigungsstrategien zum Umgang mit der eigenen Rolle wurde auch durch hohe Erwartungen der Studierenden selbst erschwert. Da sich Studierende weniger an Personen orientierten, die nicht ihren Erwartungen entsprachen, blieb die Möglichkeit des Lernens an Negativbeispielen teilweise ungenutzt.

\section{Handlungsstrategien}

Die Studierenden versuchten, insbesondere durch aufmerksame Beobachtung relevante Eigenschaften für die Bewältigung des Studiums zu erwerben und sich so auf die zukünftige Rolle als Ärzte vorzubereiten. Dabei registrierten sie aufmerksam die Reaktionen ihres Umfeldes und nahmen auch kurze Interaktionen mit Dozenten als Hinweise oder sogar Grundlage für Interpretationen von ärztlichen Verhaltensweisen. S04 z.B. nutzte die beobachtete Interaktion zwischen einem Professor und seiner Frau, um Rückschlüsse auf dessen Sozialverhalten und Persönlichkeit zu ziehen (vgl. S04 091). Wie im Kapitel 5.2.1:Orientierungshilfen durch Vorbilder: Die Rolle der Kommilitonen beschrieben, waren sowohl Lernstrategien als auch Verhaltensempfehlungen für das eigene Wohlergehen (z.B. der Umgang mit Stress) von Mitstudierenden eine wichtige Orientierungshilfe für die Betroffenen. Auch Handlungsstrategien in Bezug auf den Umgang untereinander z.B. im Rahmen von Lerntreffen wurden genau beobachtet und teilweise übernommen. Dazu gehörte es, sich unter Kommilitonen zu verbünden und sich gegenseitig zu ermutigen, aber auch zu vergleichen und zu konkurrieren. Anforderungen gemeinsam zu bewältigen und sich 
Ergebnisse

gegenseitig zur Leistung anzuspornen, schien zu motivieren und die Bewältigung zu erleichtern.

Für das Arzt-Werden waren Ärzte als Orientierung, v.a. im Rahmen von Praktika, wichtig (vgl. Kapitel 5.2.2: Orientierungshilfen durch Vorbilder Die Rolle von Lehrenden im Studium und für den Beruf). Neben einer impliziten Vermittlung von Professionalität und Medizinkultur im Rahmen des hidden curriculums, bei der Studierende eher eine passive Rolle übernahmen, wurde Professionalität und die Rolle als angehende Ärzte von ihnen auch aktiv erarbeitet. Dazu suchten die Studierenden gezielt nach Personen, von denen sie sich erhofften, eine bestimmte Hilfestellung zu erhalten oder eine für sie relevante Fähigkeit zu lernen. Auch eine kritische Auseinandersetzung mit Vorbildern oder die Ablehnung von Verhaltensweisen und die Modifizierung des eigenen Verhaltens erschlossen aktiv Wissen bezüglich der zu bewältigenden Anforderungen und zu erfüllenden Rollen.

Die gezielte Auswahl von Personen für eine Vorbildfunktion richtete sich auf Personen, die „etwas“ besaßen, das Studierende für wichtig in Bezug auf sich persönlich oder die Bewältigung des Studiums ansahen. Ein Beispiel ist die Übernahme einer, bei Kommilitonen beobachteten, „kämpferischen“ Einstellung zur Bewältigung des Lernstoffs. Wissen von anderen zu „übernehmen“, stellte ein zentrales Thema in den Berichten der Studierenden dar. Lernpartner wurden auch nach Effektivität ausgewählt.

Die Studierenden schienen sich teilweise ihrer „skrupellosen Nutzung“ von Kommilitonen bewusst zu sein. Einige wirkten beschämt wegen der passiven Art, in der sie Wissen von anderen übernahmen (vgl. S14 039,047 und 055). In dem Zusammenhang wurde von „zweckmäßige[m] Beisammensein“ mit Kommilitonen gesprochen (S12 15).

Auf der Suche nach „Identifikationsmustern“ für die künftige professionelle Rolle erprobten die Studierenden zum Teil Verhaltensweisen, die sich nicht an realen Vorbildern orientierten, sondern aus eigenen Vorstellungen eines „korrekten ärztlichen Verhaltens" erwuchsen.

\section{Konsequenzen}

Die Studierenden erprobten vielfältige und individuelle Anpassungs- und Bewältigungsstrategien - nicht immer erfolgreich. Auch wenn ihnen die Handlungsstrategien 
Ergebnisse

teilweise nicht bewusst waren, können sie trotzdem als gezielt bezeichnet werden, da im Regelfall mit einer Intention verknüpft. Aus den Begegnungen mit Kommilitonen resultierte daraus häufig ein ,Erfahrungstransfer' mit Übernahme konkreter Strategien, z.B. Lernstrategien. Auch wenn die Beschäftigung mit dem Arzt-Werden für viele noch weit entfernt war, eröffneten Kontakte zu Lehrärzten Wissen über unausgesprochene Verhaltensregeln im medizinischen Umfeld und Verständnis für „professionelles Verhalten“. Wichtig war auch die direkte Erfahrung eines „menschlichen“ Verhaltens innen gegenüber: Das machte Studierenden die Bedeutung dieses Verhaltens gerade gegenüber Patienten bewusst.

Anpassungsstrategien schienen jedoch nicht immer erfolgreich zu sein; wenn man sich nicht mit der beobachteten Medizinkultur identifizieren und eigenes Verhalten dementsprechend anpassen konnte. Dies führte teilweise zu Konflikten mit professionellen Rollen, zu Isolations- und Insuffizienzgefühlen (vgl. Kapitel 5.2.3: Identifikation mit Ärzten, Studium und Beruf und 5.2.5: Rolleneinübung: Auseinandersetzung mit der Rolle als Medizinstudent/in und Antizipation der Arztrolle) und zu mangelnder Motivation für den späteren Beruf. Hilfreich waren bzw. bewundert wurden dagegen Ärzte, die immer wissen, was sie „zu tun“ haben (vgl. S13 052). Solche Ärzte fungierten für S13 als Vorbilder. Von dem Kontakt zu innen schien er sich zu erhoffen, selbst ähnliche Handlungskompetenzen zu erwerben und ihre "Sicherheit" im Umgang mit schwierigen Situationen zu übernehmen.

Da Bedürfnisse nach Unterstützung und Orientierung bei den Studierenden unterschiedlich ausgeprägt waren, fielen auch gezielte Handlungsstrategien wie die Kontaktaufnahme mit bestimmten Dozenten unterschiedlich aus.

Abbildung 8 fasst die Ergebnisse zum Thema „Entwicklung von Strategien zur Bewältigung des Studiums" im Sinne des Kodier-Paradigmas zusammen: 


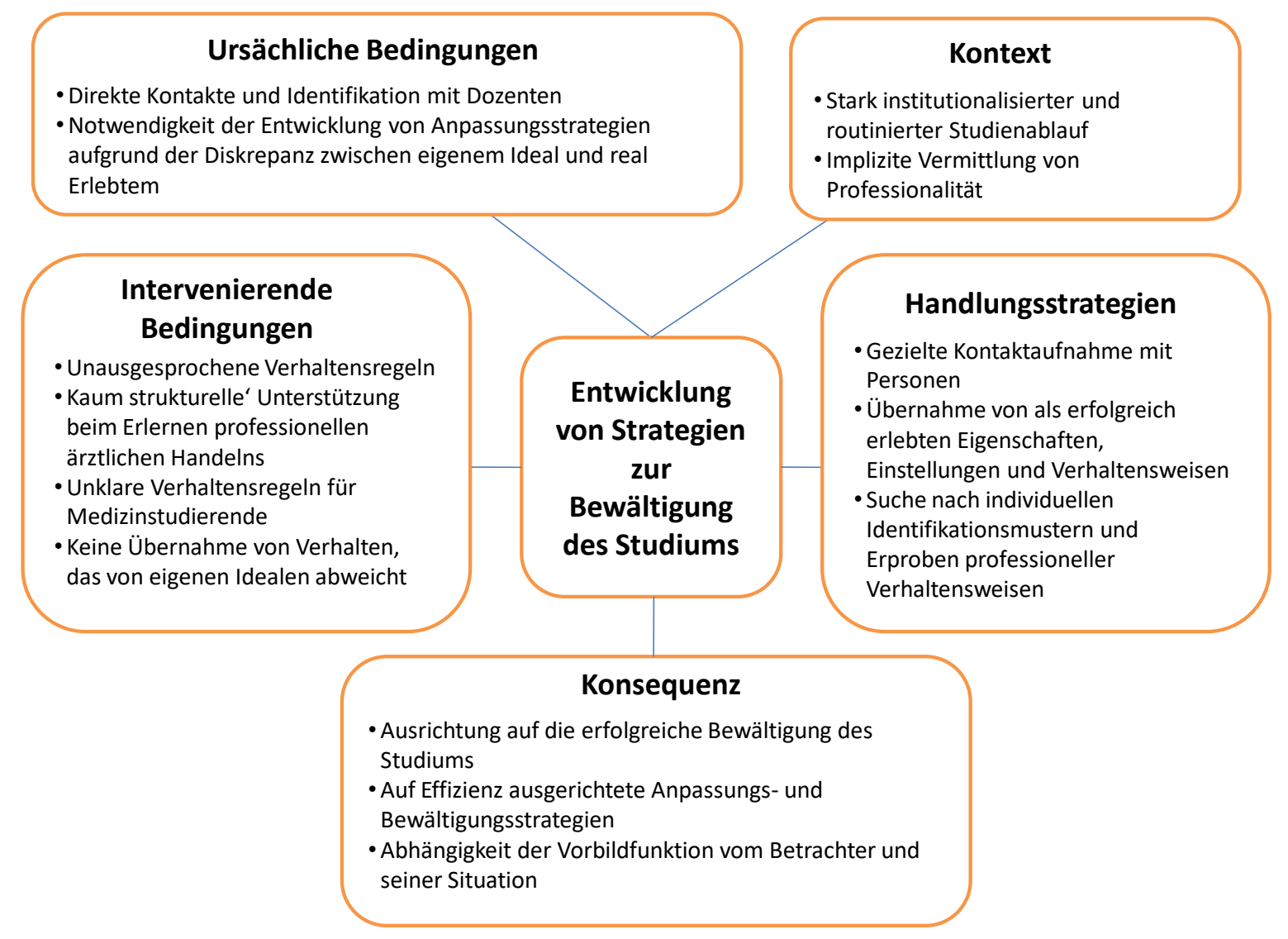

Abbildung 8: Kodier-Paradigma zum Thema „Entwicklung von Strategien zur Bewältigung des Studiums“. 


\section{Diskussion}

\subsection{Zusammenfassung der Ergebnisse}

Wenn Studierende der Medizin Ärzte vor allem in deren Arbeitsroutine erleben, beginnen sie, ihre zukünftige Rolle zu antizipieren. Sie können sich an Ärzten orientieren, sich mit innen identifizieren und schon früh mit den Idealen der ärztlichen Profession auseinandersetzen. Das ist aus anderen Studien hinlänglich bekannt, findet sich in der vorliegenden Studie bestätigt und macht auf die Bedeutung von Vorbildern und deren Wirkung erneut aufmerksam. Zugleich konnten wir herausarbeiten, dass das Erleben von Vorbildern und die Auseinandersetzung mit innen Strategien zur Bewältigung des Studiums und der Einübung der Arztrolle bahnen. Studierende suchten und erprobten aktiv Strategien, mit deren Hilfe sie das neue medizinische Umfeld als neuen Lebenskontext erschlossen. Funktion und Wirkung von Vorbildern ist also mehr als ein passiver Prozess der Einwirkung auf Novizen. Eine weitere wichtige Erkenntnis schloss sich an: Vorbildfunktionen übernahmen nicht nur Akteure, die bewusst und typischerweise als Vorbilder wahrgenommen werden bzw. gelten, sondern auch Personen im engeren Umfeld, v.a. Freunde, Kommilitonen oder Pflegende. Wirkung und Funktion von Vorbildern hingen weniger von den Akteuren (zumeist beobachteten Ärzten), sondern vielmehr von den Rezipienten (den Studierenden), ihrer Situation und ihren subjektiven Bedeutungszuschreibungen und Definitionen des Begriffs ,Vorbild“ ab.

Nicht alle Forschungsfragen konnten abschließend beantwortet werden. Umgekehrt führte die Analyse der Interviews zu Ergebnissen, die jenseits der anfänglichen Forschungsfragen liegen, z.B. der Einfluss der aktuellen Situation der Studierenden auf die Funktion und Wirkung von Vorbildern. Diesen ,strukturellen' Bedingungen der Interaktion zwischen Studierenden und Vorbildern soll in der Diskussion besonders Raum gegeben werden. Die Diskussion orientiert sich an den Themen, die Studierende tatsächlich in den Mittelpunkt stellten (ein Beispiel sind negative Ereignisse).

Der Aufbau der Diskussion folgt Abbildung 9 (s.u.), die - im Sinne des KodierParadigmas - die Ergebnisse im strukturellen Überblick zeigt. Dem Charakter einer qualitativen Methode folgend, stehen dabei wiederum die sich in den Interviews ausdrückenden Erfahrungen und Sichtweisen im Vordergrund, jeweils ergänzt - oder 
Diskussion

auch kontrastiert - mit den Ergebnissen der quantitativen Umfrage. Durch die Gesamtschau der Ergebnisse und Berücksichtigung der einschlägigen Literatur soll das zentrale Thema "Vorbilder und prägende Personen im Medizinstudium“ in seinen Bedingungen, im Kontext, in seinen Konsequenzen und Handlungsstrategien nachvollziehbar werden.

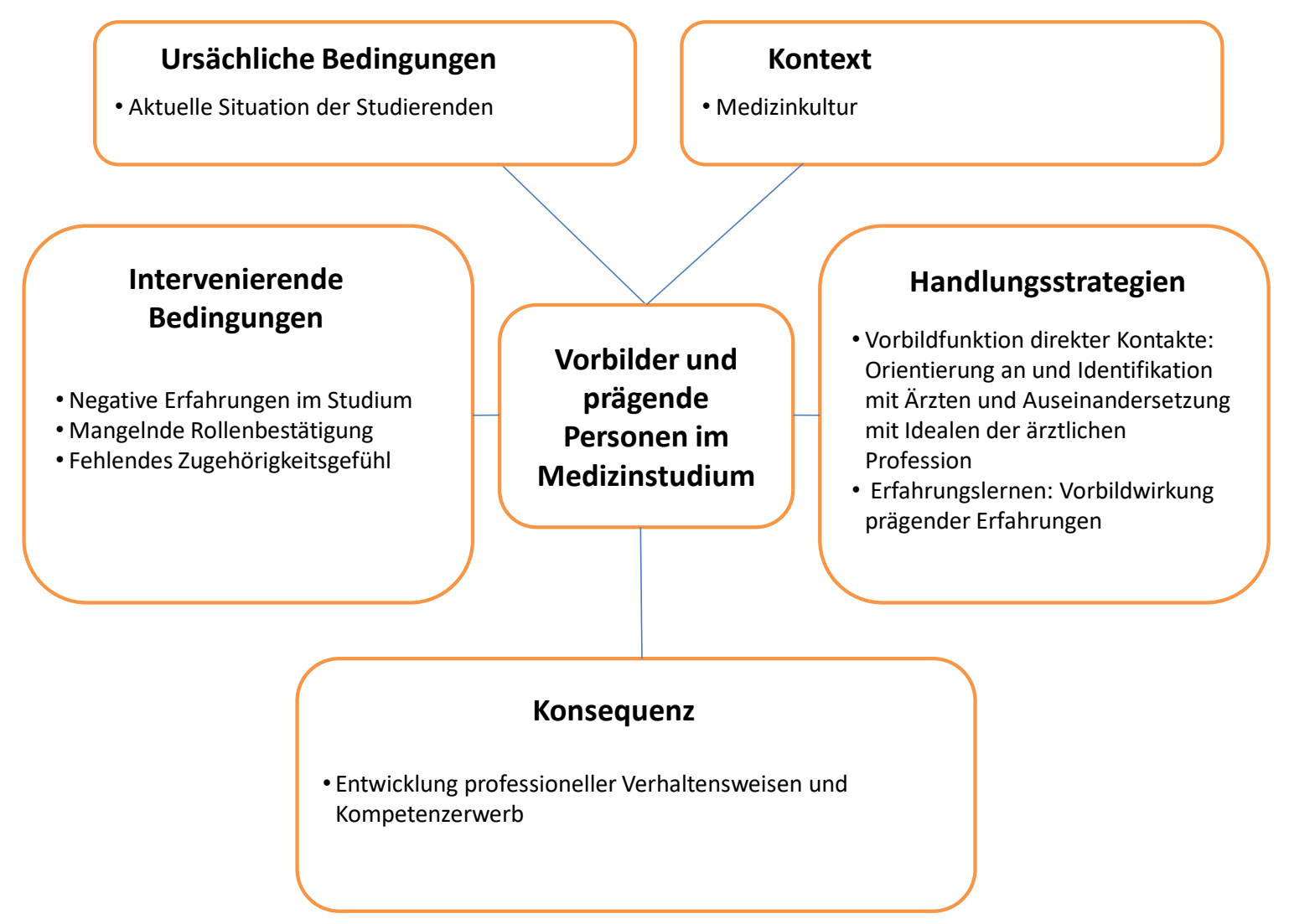

Abbildung 9: Zusammenfassendes Kodier-Paradigma zum Dissertations-Thema „Vorbilder und prägende Personen im Medizinstudium".

\subsection{Vergleich mit der Literatur}

In der Literatur wird der Begriff ,Vorbild' auf unterschiedliche Weise definiert und verwendet. Einige Autoren verzichten gänzlich auf eine Begriffsklärung. Der daraus resultierende Interpretationsspielraum erschwert die Bewertung und den Vergleich der Studien untereinander. Diese Studie hatte das Ziel nachzuvollziehen, wie Studierende der Medizin Vorbilder wahrnehmen und erleben und welche Bedeutung Vorbilder für sie heute haben. Hier fiel zunächst die große Variation auf, wie bereits die Themen der folgenden Kapitel andeuten. 


\subsubsection{Schilderung negativer Erfahrungen}

In Freitexten der ELSAS-Studie (Hilbert 2012), die als erster Schritt für die vorliegende Arbeit aufbereitet wurden, war der Begriff ,Vorbild' meist mit hohen Idealen verknüpft. Die Äußerungen der Studierenden aus der ELSAS-Studie waren im Vergleich zu den neu geführten Interviews überwiegend positiv. Auch in der Literatur werden Vorbilder zumeist positiv definiert. In den Interviews - der zweite Schritt der vorliegenden Arbeit - wurden hingegen auch negative Erfahrungen mit Personen berichtet, die wie Anti-Vorbilder beschrieben wurden. Dies entspricht Berichten über die unangemessene Behandlung von Studierenden im Rahmen der medizinischen Ausbildung (vgl. Baldwin et al. 1988; Shuval und Adler 1980; Weissmann et al. 2006; Asghari et al. 2011); Gágyor et al. 2012).

Solche negativen Erfahrungen sind aber nicht unbedingt ein Anstoß, es als zukünftige Ärzte besser zu machen: Von Anti-Vorbildern könne durch unreflektiertes Nachahmen ihres Verhaltens auch ein negativer Einfluss ausgehen (vgl. Benbassat 2014). Schrauth et al. (2009) sehen es als wahrscheinlich an, „dass im Laufe der Sozialisation das Selbstbild eher dem realen Arztbild als dem Idealbild angenähert wird“ (Schrauth et al. 2009).

Eine mehrfach erwähnte und belastende negative Erfahrung für Studierende in unserer Studie war die fehlende Beachtung und Betreuung durch Dozenten. Auch in der ursprünglichen ELSAS-Auswertung von Nadine Hilbert war das Übergehen oder Nichtbeachten von Medizinstudierenden eine häufige negative Erfahrung (sie wurde von $17,1 \%$ berichtet und war damit die dritthäufigste negative Erfahrung). Unsere Ergebnisse legen die Vermutung nahe, dass besonders ein Mangel an Unterstützung und Reflexionsangeboten durch erfahrenere Mitglieder der Fakultät Studierende belastet und negativ prägt (s. Kapitel 6.2.3: Einfluss der aktuellen Situation der Studierenden auf die Funktion und Wirkung von Vorbildern).

\subsubsection{Medizinkultur und implizites Lernen}

Mit dem Einfluss der „Medizinkultur“24 auf Studierende beschäftigten sich u.a. bereits Maudsley 2001, Benbassat 2014, Kenny et al. 2003 oder Wright et al. 1998). Für un-

\footnotetext{
${ }^{24}$ Als ,Medizinkultur' werden hier Werte, Normen oder kulturelle Muster verstanden, die von zugehörigen Personen geteilt werden und professionsspezifisches Handeln ermöglichen und gestalten vgl. Fußnote 3 und 23.
} 
Diskussion

ser Thema sind vor allem Studien zum Einfluss des hidden curriculum, die die Bedeutung informellen, impliziten ${ }^{25}$ Lernens betonen, von Bedeutung (vgl. Hafferty und Franks 1994; Gaufberg et al. 2010). Auch in der vorliegenden Studie gab es deutliche Hinweise darauf, dass Informationen über den späteren Beruf und damit verbundene Anforderungen v.a. latent vermittelt werden. „Unausgesprochene Gebote“ beispielsweise, die erwünschtes Verhalten markieren, wurden von den Studienteilnehmer/innen häufig beschrieben. So lässt sich aus unseren Ergebnissen auch für die medizinische Ausbildung an deutschen Universitäten der Schluss ziehen, dass die im ,Kulturraum Medizin ${ }^{26}$ vorherrschenden Wahrnehmungs-, Deutungs- und Handlungsmuster großen Einfluss haben und einen bedeutsamen Sozialisationsraum für heranwachsende Ärzte schaffen (Becker 1977). Über die Auseinandersetzung mit ärztlichen Vorbildern als Teil des Kulturraums werden nicht nur persönliche Ideale und Werte vermittelt, sondern auch diejenigen der Profession und der medizinischen Kultur. Dass hierbei auch der Austausch von Studierenden untereinander eine Rolle spielt, konnten wir mehrfach zeigen. Auch andere Autoren stellten fest, dass Unterhaltungen unter Studierenden, bei denen keine Fakultäts-Mitglieder anwesend sind, die Mehrheit der wichtigen informellen Interaktionen ausmachen (Becker 1977; Hundert et al. 1996).

Studierende bewegten sich allerdings in diesem Raum auch aktiv und strategisch, um ihre Rolle einzuüben und zu erfüllen: ein fortlaufender Prozess von Anpassung, Distanzierung und Modifizierung des Verhaltens in Bezug auf die "Medizinkultur" in der Interaktion mit ihren Mitgliedern (vgl. Kapitel 5.2.5 Rolleneinübung: Auseinandersetzung mit der Rolle als Medizinstudent/in und Antizipation der Arztrolle). Dieses Ergebnis deckt sich mit denen einer frühen Studie von Shuval und Adler (1980) zur Rolle von Vorbildern für die professionelle Sozialisation von Medizinstudierenden. Sie beobachteten aktive Identifikation wie auch Ablehnung und passive Muster als Interaktionsformen mit Vorbildern und zeigten, dass Studierende Vorbilder selektiv auswählen, alternative Vorbilder in Erwägung ziehen und einige Lehrärzte auch als schlechte Vorbilder betrachten, die damit auch eine Orientierungsfunktion hatten.

\footnotetext{
${ }^{25}$ Zum Begriff ,implizit‘ vgl. Fußnote 2.
} 
Diskussion

Ähnliche Verhaltensweisen zeigten ebenfalls die Teilnehmer/innen in der vorliegenden Studie. Sie bemühten sich jedoch auch, die Erwartungen ihres Umfeldes zu erfüllen, indem sie sich an Anforderungen des neuen Kulturraumes anpassten und z.B. versuchten, nicht negativ aufzufallen. Aktive Bewältigungs- und Handlungsstrategien zu entwickeln bedeutete somit nicht, dies losgelöst und unabhängig vom Umfeld und von vorherrschenden Verhaltensnormen zu tun. Ob eine Anpassung an Normen und die „Konformität" mit der eigenen Rolle als Medizinstudierende möglich war, schien auch davon abzuhängen, ob Anforderungen durch das Umfeld klar vermittelt wurden. Dies setzte u.a. die Möglichkeit des Kontaktes mit erfahrenen Mitgliedern des Kulturraumes wie Ärzten voraus, was häufig nicht gegeben zu sein schien.

\subsubsection{Einfluss der aktuellen Situation der Studierenden auf die Funktion und Wirkung von Vorbildern}

Die Studie zeigt Zusammenhänge zwischen der Situation der Studierenden, der Suche nach Vorbildern und deren Wirkung. Die Teilnehmer/innen dieser Studie beschrieben Unsicherheit, Verlorenheitsgefühle, Orientierungslosigkeit und Zweifel am eigenen Können insbesondere zu Beginn des Studiums. Dies wurde auch durch andere Untersuchungen bestätigt. Nach Schrauth et al. (2009) ist das Selbstbild von Medizinstudierenden maßgeblich von Gefühlen der Machtlosigkeit und Unsicherheit geprägt. Sich nicht in der eigenen Rolle als "Lernende" akzeptiert und eingebunden zu fühlen, erschwerte den Teilnehmern dieser Studie eine Rolleneinübung. Den eigenen Platz im medizinischen Umfeld $\mathrm{zu}$ finden und das Studium zu bewältigen, stand im Mittelpunkt ihrer Handlungen und Anstrengungen, verbunden mit der Suche nach Hilfe und Unterstützung.

Prägende Personen und die Erfahrungen mit direkten Vorbildern fungierten häufig als Unterstützer; sie stärkten das Selbstbewusstsein, motivierten, eröffneten Zukunftsperspektiven für den angestrebten Beruf und vermittelten Sicherheit und Zugehörigkeit durch Akzeptanz. Die Analyse zeigte, wie diese Personen als ,Bewältigungsstrategien' für das Studium und der Erarbeitung einer professionellen Rolle

${ }^{26}$ Der Begriff ,Kulturraum Medizin' wurde bereits im Ergebnisteil eingeführt (vgl. Kapitel 5.2.5: Rolleneinübung: Auseinandersetzung mit der Rolle als Medizinstudent/in und Antizipation der Arztrolle). Die hier aufgeführten Überlegungen zum Einfluss des Kulturraums auf Studierende sind an eine kultursoziologische Sichtweise angelehnt, die die kulturelle Bedingtheit sozialer Erscheinungen betont (vgl. Moebius und Albrecht (2014); Wiegelmann (1980)). 
dienten: nämlich als ,Informationsquellen' über informelle Kriterien der Ausübung der Rolle als Ärzte und akzeptierter Verhaltensweisen im Kulturraum Medizin. Im Kontext einer oft als mangelhaft wahrgenommenen Unterstützung von Seiten der Fakultät entwickelten die Studierenden Strategien, um auch unabhängig von bestehenden Strukturen agieren zu können.

\subsubsection{Die Rolle von Anti-Vorbildern für die Entwicklung professioneller Ver- haltensweisen}

Eine wichtige Handlungsstrategie war die bewusste Distanzierung von Negativbeispielen. Im Rahmen der Interviews reflektierten die Studienteilnehmer/innen kritisch über das Verhalten ihrer Dozenten. Goffmann (1973) spricht im Zusammenhang mit dem Professionalisierungsprozess auch vom Erlernen einer „Rollendistanz“, die eine distanzierte Betrachtung von Erwartungen ermöglicht, die mit der eigenen Rolle verbunden sind. Auch Adler und Shuval (1980) sahen die zunehmende Neigung der Studenten, Botschaften nach unabhängigen Kriterien auszuwählen, als den wichtigsten Einfluss auf die Sozialisierung Erwachsener. Dazu gehöre es, Botschaften auf ihre Angemessenheit hin zu überprüfen und zu selektieren, ggf. auch zu ignorieren. Verhalten orientiert sich somit nicht nur an Vorbildern, sondern auch an AntiVorbildern. Dies zeigen auch unsere Ergebnisse.

\subsubsection{Vorbildwirkung im direkten Kontakt}

Die Beziehung zum Vorbild bauten Studierende auf zwei grundlegend unterschiedliche Arten auf: Zum einen wurden (indirekte) Vorbilder aus der Distanz heraus beobachtet und ihr Auftreten und ihre Person als Verhaltensideal bewundert. Solche Vorbilder wurden häufig wegen ihres Vorsprungs an Wissen und Erfahrung als überlegen wahrgenommen. Dozenten, zu denen eine Distanz bestand, setzten durch ihr Auftreten und Handeln Verhaltensmaßstäbe und vermittelten damit Inhalte der Medizinkultur. Sie wirkten auf Studierende jedoch eher wie ein fernes Ziel, von dem sie sich in ihrer jetzigen Situation zu weit entfernt fühlten, um es überhaupt anzustreben. Sie vermittelten den Studierenden somit zwar Ideale, z.B. in Bezug darauf, was sie wissen sollten, halfen ihnen in ihrer aktuellen Situation und mit der Entwicklung ihrer professionellen Rolle jedoch wenig.

Zum anderen dienten Personen als Vorbilder, die im direkten Kontakt für ihren zwischenmenschlichen Umgang und für ihre „Persönlichkeit“ bewundert wurden. Dozen- 
Diskussion

ten, deren Beziehung zu den Studierenden durch Nähe gekennzeichnet war, gaben den Studierenden die Möglichkeit, ihr 'Arzt-Sein' direkt zu erleben und durch ihre Offenheit persönliche Erfahrungen und Einstellungen zu Inhalten des Berufs zu erfahren. Wenn sich diese mit eigenen Zielen deckten, zeigten Vorbilder den Studierenden, dass diese erreichbar sind und es sich lohnt, darauf hinzuarbeiten. Solche „direkten" Vorbilder wirkten gerade durch ihre persönliche Seite. So war es den Studierenden auch möglich, sie "als Menschen“ kennen zu lernen und von ihnen Eigenschaften zu lernen, die nur im unmittelbaren Kontakt möglich sind, z.B. Empathie, die sich somit auf die Interaktion mit Patienten übertragen ließen. Burkart (1983) schrieb in diesem Zusammenhang: „Regeln [ärztlichen Verhaltens] lassen sich nur dann vermitteln, wenn sie nicht in abstrakter Form formuliert werden, sondern am Einzelfall demonstriert werden“. Anders gesagt: Eine Lehrveranstaltung über Empathie macht nicht empathisch, Empathie jedoch in einer Begegnung selbst zu erfahren, hat dieses Potential (vgl. Böhle et al. 2008).

Von Vorbildern erwarteten die Studierenden auch ,etwas in Bezug auf sie persönlich': ob sie z.B. auf sie eingingen und ob sie Studierende an Begegnungen mit Patienten teilnehmen ließen. Die Studierenden waren dann nicht mehr nur Beobachter, sondern Interaktionspartner in einer Beziehung zum Vorbild. Direkte Kontakte beinhalteten eine Beziehungskomponente, die eine Identifikation erleichterte. Erst die Nähe zu Dozenten gestattete es den Studierenden, sich im Vorbild zu sehen'. Distanz im Verhältnis zu Dozenten schuf zwar Respekt und hatte ebenfalls eine Vorbildwirkung, erschwerte jedoch eine Identifikation. Dafür schien die Kenntnis der Dozenten „als Person" Voraussetzung zu sein. Dies betont die Wichtigkeit der Interaktion und Teilhabe in Bezug auf das Lernen am Vorbild.

In der Beschreibung der Studierenden vermischen sich teilweise Erwartungen an professionelle und persönliche Vorbilder. Da nicht nur Erwartungen an Vorbilder als Rollen-Vorbilder, sondern auch als „persönliche Vorbilder“ bestanden, wurde die Suche nach Personen erschwert, die diese in sich vereinten.

\subsubsection{Vorbildfunktion prägender Erfahrungen}

Nicht nur die Beziehung zu einer Person konnte Vorbildfunktionen für die Studierenden haben. Häufig nahmen sich die Studierenden Teilaspekte von Personen zum Vorbild und orientierten sich an unterschiedlichen Personen. Der Wert des ,Erfah- 
Diskussion

rungslernens ' wird durch Studien belegt, die den Einfluss von Erfahrungen in direkten Begegnungen mit Patienten auf die Entwicklung von Studierenden hervorheben (vgl. u.a. Cruess 2012). Ist dann eine Vorbildwirkung überhaupt noch an Personen gebunden? Das ,Konstrukt Vorbild' würde vielmehr ein ,Set an bestimmten Eigenschaften' beinhalten, die das Vorbildliche ausmachen. Das schlägt die Brücke zum Behaviorismus, wonach erlernte Verhaltensweisen auf Erfahrungen basieren. Ob erste Erfahrungen im medizinischen Umfeld positiv oder negativ erlebt wurden, schien entscheidend zu beeinflussen, an welchen Verhaltensmaßstäben sich Studierende zukünftig orientierten. Wurden Erfahrungen mit Personen als positiv wahrgenommen, trugen sie zu mehr Sicherheit und einem größeren Selbstbewusstsein in Bezug auf die eigene Rolle bei, zu einer Identifikation mit dem Studium und dem angestrebten Beruf und dem Gefühl, ,auf dem richtigen Weg' in die Rolle als Arzt/Ärztin zu sein. Dann wäre die Erfahrung prägender als die daran beteiligten Personen im Sinne von Vorbildern.

\subsubsection{Lernen von Kommilitonen: mehr als nur Erfahrungslernen}

Ähnlich der Beziehung zu Ärzten spielte auch für die Vorbildfunktion von Kommilitonen Vertrauen, fehlende Angst und eine als gleichberechtigt empfundene Beziehung eine große Rolle. Kommilitonen sind für unser Thema auch deshalb interessant, da sie in der medizinischen Hierarchie nicht höher stehen und kein Lernen von Vorbildern im traditionellen Sinne stattfindet, bei dem der Meister seinem Lehrling kompetentes Handeln im Vollzug demonstriert. Am Beispiel der Kommilitonen lassen sich somit ,unkonventionelle' Vorbildfunktionen feststellen, die zu einem erweiterten Verständnis der Funktion von Vorbildern führen: Im Idealfall gewähren Kommilitonen beides, das Lernen durch gemeinsame Erfahrungen und partiell bzw. sukzessiv die Orientierung an innen und die Übernahme ihrer Verhaltensweisen oder Haltungen. Sie können also bei dem Erwerb von Kompetenzen und der Ausstattung mit Schlüsselkompetenzen sowohl als Vorbilder dienen, die durch das eigene Beispiel demonstrieren, welche Eigenschaften im Studium und für den späteren Beruf von Vorteil sind, als auch im Rahmen von gemeinsam gemachten Erfahrungen das Lernen unterstützen.

Zur Vermittlung professioneller Handlungsweisen durch Vorbilder schrieb Burkart (1983), der Erwerb von Schlüsselkompetenzen sei an die Teilnahme an Handlungssituationen gebunden, in denen ein kompetentes Professionsmitglied („der Meister“) 
Diskussion

Verhaltensweisen demonstriert. Erst dadurch bestehe die Möglichkeit, die Struktur der professionellen Handlungskompetenz zu erfassen (vgl. Parsons 1958). Kommilitonen wirken im Sinne von Hierarchien nicht als ,Meister'. So waren sich die Studierenden häufig auch ihrer Vorbildfunktionen nicht bewusst und erkannten Kommilitonen nicht als Vorbild an, schien doch die Vorbildfunktion oft mit einem ,Vorsprung' an bestimmten Kenntnissen oder Erfahrungen verbunden zu sein. Dies schien mit dem Ausbildungsstand zusammenzuhängen, wurden doch ältere Kommilitonen offen für ihr größeres Wissens und auch die Tatsache bewundert, bereits einen größeren Teil des Studiums geschafft zu haben.

Dass auch Kommilitonen und Freunde im gleichen Semester Vorbildfunktionen erfüllen, erstaunt. Die Orientierung an innen und die Übernahme auch ihrer Verhaltensweisen scheint insbesondere zur Bewältigung des Studiums sinnvoll zu sein. Sie trugen in besonderer Weise zum Erfahrungslernen bei, da mit ihnen ein direkter ,Erfahrungsaustausch und Wissenstransfer' stattfand. Durch eigene Erfahrungen (z.B. in Praktika) erlangtes Wissen und ,vorteilhafte' Verhaltensweisen wurden weitergegeben und ausgetauscht. Erfahrungen in Lerngruppen spielten nicht nur in Bezug auf die Erweiterung des Fachwissens eine Rolle. Der Erlebnisaustausch erweiterte das gemeinsame Wissen über Normen und Verhaltensweisen des Kulturraums Medizin. Kommilitonen waren nicht nur in ihrer Lehrfunktion und durch größere Kenntnisse Vorbilder, sondern z.B. auch aufgrund bestimmter Charaktereigenschaften wie Hilfsund Kooperationsbereitschaft oder ihrer Freundlichkeit. ,Am Vorbild“ lernten Studierende den Umgang mit dem Studium wie Prüfungsvorbereitung, Zeitmanagement, Stressbewältigung und Selbstfürsorge. In diesem Punkt ist ihre Funktion vergleichbar mit der Orientierungshilfe, die sie durch Ärzte erfuhren. Ein gemeinsames Erfahrungslernen im Studium wurde jedoch darüber hinaus dadurch ermöglicht, dass Kommilitonen wie ,Verbündete' erlebt wurden, die auf der gleichen Seite stehen und ein Verständnis für ihre Probleme im Studium haben. Vorbildfunktionen erfültten sie auch füreinander, wenn es darum ging, sich nicht ,unterkriegen zu lassen', den anderen Mut zu machen und durch Haltungen wie z.B. Optimismus in Bezug auf die Bewältigung von Prüfungen oder der Sinnhaftigkeit des Lernens.

\subsubsection{Kompetenz und Professionalität im Studium erwerben}

Eine weitere nicht-klassische Funktion und Wirkung von Vorbildern im Zusammenhang mit "der Professionalisierung“ der Studierenden zeigte sich v.a. auf informellem 
Diskussion

Wege. Karnieli-Miller et al. (2010) fanden heraus, dass Studierende Professionalität und professionelle Werte kontinuierlich, also während ihres gesamten Studiums, lernen. Die Auswertung der ELSAS-Daten macht deutlich, dass „Professionalität“ als zentrale ärztliche Eigenschaft angesehen und Ziel ihrer Ausbildung gesehen wurde. „Professionalität" als Eigenschaft von Ärzten schien für die Teilnehmer/innen in der ELSAS-Studie ein wichtiges Kriterium zu sein, um von deren Vorbildfunktion zu sprechen. Dieses Ergebnis deckt sich mit den Ergebnissen der Interviews, wonach die Studierenden das Erlernen professioneller Verhaltensweisen im Studium für wichtig hielten. Sie bewunderten vor allem Ärzte, die die unterschiedlichen mit dem Beruf verbundenen Rollen souverän erfüllten.

Was im Einzelnen als professionelles Verhalten angesehen wurde, unterschied sich stark. Dies mag zum Teil auf das persönliche Wertesystem der Studierenden zurückführbar sein. Es kann aber auch als Hinweis darauf gewertet werden, dass die Fakultät wenig Vorgaben macht und professionelle Eigenschaften nicht Teil des offiziellen Curriculums sind (ähnlich auch Kenny et al. 2003). Ärzte, die für ihr Verhalten besonders geschätzt oder bewundert wurden, konnten eine Hilfe bei der Rolleneinübung sein bzw. eine orientierende Funktion haben, ohne aber das häufig mit einer Vorbildfunktion verbundene Idealbild zu verkörpern. Solche ,indirekten' Vorbilder gaben erste Einblicke in die zukünftige Rolle und ein konkreteres Bild des Arztberufes, das jedoch häufig nicht positiver war als das vorgefertigte Idealbild (so auch Schrauth et al. 2009).

Im Zusammenhang mit ihrer Professionalisierung scheint es ebenfalls von Bedeutung zu sein, Studierende zur Reflexion zu ermutigen (vgl. Jarvis-Selinger et al. 2012). Den Ergebnissen dieser Studie zufolge geschieht derzeit eine Reflexion und eine Auseinandersetzung mit Idealen, wenn überhaupt, im Austausch mit anderen Studierenden, aber ohne eine Einladung von offizieller Seite. Kenny et al. (2003) betonten die Bedeutung von Reflexion für die Integration persönlicher Werte und Einstellungen in eine „professionelle Kultur“. Ihre Effektivität werde durch die Unterstützung von Mentoren und Vorbildern erhöht (vgl. u.a. Weissmann et al. 2006; JarvisSelinger et al. 2012). 


\subsubsection{Mangelnde Rollenbestätigung und Zugehörigkeit}

Viele Studierende fühlten sich nicht ausreichend in ihrer Rolle als "Lernende“ akzeptiert. Gerade dies schien jedoch ein entscheidendes Kriterium dafür zu sein, ob sich Studierende mit ihrem Studium und auch mit der Rolle als angehende Ärzte identifizieren konnten (vgl. Kapitel 5.2.3: Identifikation mit Ärzten, Studium und Beruf).

Die Bedeutung des Zugehörigkeitsgefühls für die professionelle Sozialisation von Medizinstudierenden hoben Cruess et al. (2015) hervor. Die wichtigsten Faktoren für die Entwicklung einer professionellen Identität sind danach Vorbilder und Mentoren und die Sammlung individueller Erfahrungen. Im Vergleich zum amerikanischen Ausbildungsmodell wird der Weg in die "medizinische Gemeinschaft" in Deutschland von offizieller Seite wenig gebahnt (vgl. Cruess et al. 2015). Die traditionelle Beschränkung auf das rein Fachliche und die "inhaltlich-kognitive" Ausrichtung von Lehrveranstaltungen an deutschen medizinischen Fakultäten ist bekannt (vgl. Witte 2010). Auch unsere Studie bestätigt, dass das Wissen um professionelle Verhaltensweisen und deren Einübung vor allem ,unterschwellig'vermittelt werden, dies bleibt der Eigeninitiative der Studierenden überlassen. Die Entwicklung professioneller Verhaltensweisen und Sicherheit im Umgang mit der eigenen Rolle zu erreichen, waren jedoch den Studienteilnehmer/innen sehr wichtig. Implizit wird nämlich - so ihr Eindruck - erwartet, dass sie sich z.B. während klinischer Praktika „professionell“ verhalten. So fühlten sie sich beispielsweise dazu verpflichtet, bereits im Studium kompetent zu wirken - und dabei allein gelassen.

Das Gefühl, mit der Erarbeitung eines professionellen Verhaltens und der Bewältigung des Studiums allein gelassen zu werden, ließ Studierende aktiv nach Strategien suchen, wie man beispielsweise durch gezielte Kontaktaufnahme Informationen gewinnt, um das eigene Wissen zu erweitern. Witte (2010) schrieb, dass professionelle Sozialisation vor dem Hintergrund mangelnder Unterstützung von offizieller Seite zur persönlichen Aufgabe von angehenden Ärzten wird. Unsere Studie zeigte, dass unter diesen Bedingungen die individuelle Orientierung an Mitgliedern der Fakultät durch direkte Kontakte an Bedeutung gewinnt. Dabei orientierten sich Studierende nicht nur an Verhaltensweisen, die in Bezug auf die bevorstehende Rolle als Ärzte offensichtlich Vorbildfunktion haben, z.B. das Verhalten Patienten gegenüber, sondern auch am Verhalten anderen Berufsgruppen und v.a. den Studierenden gegenüber. Sie wünschten sich von Ärzten eine ähnliche Behandlung wie die, die sie 
Diskussion

auch Patienten gegenüber als wesentlich erachteten: einen respektvollen, gleichberechtigten und empathischen Umgang.

Nach Haidet und Stein (2006) sind Beziehungen zwischen Studierenden und Lehrenden wichtige Ausgangspunkte bzw. Modell für Beziehungen, wie sie Studierende später gegenüber Patienten pflegen.

Beziehungen der Dozenten zu ihren Studenten sind Schlüssel-Erfahrungen, auf die Studierende später zurückgreifen werden. Botschaften, gerade auch unbeabsichtigte Signale von prägenden Personen können einen gravierenden Effekt auf die Entwicklung von Studierenden und auf ihr Sozialverhalten als spätere Ärzte haben (vgl. u.a. Weissmann et al. 2006; Karnieli-Miller et al. 2010).

Die Bedeutung sozialer Anerkennung und Bedürfnisse nach Affiliation, Gesellschaft und Zugehörigkeit untersuchte bereits Murray (2008). Merton et al. (1957) nannten als Endpunkte professioneller Sozialisation, dass Studierende „wie Ärzte denken, handeln und sich wie Ärzte fühlen“. Cruess et al. (2012) nannten in ihrer Studie als ein entscheidendes Ziel der medizinischen Ausbildung die Entwicklung einer professionellen Identität. Ein Ziel, das vor dem Hintergrund der Ergebnisse dieser Studie weit entfernt scheint, waren die Studienteilnehmer/innen doch maßgeblich mit der Frage beschäftigt, ob sie überhaupt in die ihnen vermittelte Medizinkultur „passen“. Bei der mehrfach angesprochenen Diskrepanz zwischen eigenen Idealen und real erlebtem Verhalten von Dozenten ging es nicht nur um professionelle Verhaltensweisen mit Patienten, sondern v.a. um eine mangelnde Vermittlung von Akzeptanz und Zugehörigkeit an die Studierenden. Wenn Witte schreibt

„Es zeigte sich die Eingebundenheit des einzelnen ärztlichen Akteurs in eine professionelle Wir-Gruppe als in hohem Maße bedeutsam für (...) die berufliche Sozialisation und das konkrete berufliche Handeln" (Witte 2010),

kann im Umkehrschluss gefolgert werden, dass mangelnde Einbindung und Rollenbestätigung durch das Umfeld die Entwicklung einer professionellen Identität behindert. Dies wird von anderen Studien bestätigt (Cruess et al. 2015; Bjorksten et al. 1983; Asghari et al. 2011). Der Einfluss prägender Personen (also auch Vorbilder) auf die Entwicklung einer professionellen Identität scheint sich somit auch auf die Wahrnehmung des zukünftigen Arztberufes zu erstrecken (vgl. Schrauth et al. 2009). 


\subsection{Stärken und Schwächen der Untersuchung}

Die Studie bietet durch die zwei methodisch unterschiedlichen Zugänge einen direkten Vergleich beider Ansätze. Im zweiten Teil, den Narrativen Interviews, wurden Aspekte angesprochen, die durch eine weitgehend standardisierte Befragung nicht zum Ausdruck kommen. Auf der anderen Seite konnten einige Ergebnisse, die sich in beiden Studienteilen zeigten, gewissermaßen wechselseitig validiert werden.

Ein Grundprinzip der Grounded Theory, das ,theoretical sampling', das die Auswahl neuer Interviewpartner/innen auf der Grundlage bereits durch Auswertung gewonnener Erkenntnisse beinhaltet, konnte in der Durchführung der Interviews nicht umgesetzt werden, da für die Datenerhebung nur eine begrenzte Zeitspanne zur Verfügung stand. Es wurde sich jedoch an den Relevanzsetzungen der Interviewten orientiert und innen Raum gegeben, ihre Deutungsmuster darzustellen. Andere Gütekriterien qualitativer Forschung (Meyer et al. 2012) wie die Suche nach dem Widerspruch und das Prinzip der Offenheit wurden ebenfalls berücksichtigt. Im Zuge der Auswertung wurden hierzu zunächst alle möglichen Lesarten einer Textstelle erarbeitet. Anschließend erfolgte eine Überprüfung dieser als Hypothesen formulierter Überlegungen anhand anderer Textstellen. ,Die Suche nach dem Widerspruch' bedeutet dabei auch, nach Textstellen zu suchen, die solche Hypothesen widerlegen und zum Verwerfen einer Hypothese führten.

Die qualitative Erhebungs- und Auswertungsmethoden machte latente Sinninhalte zugänglich. So zeigte sich eine Diskrepanz zwischen dem, was Studierende bewusst an Meinungen äußerten, und dem, was innen selbst z.B. in Bezug auf ihr Verhalten nicht bewusst zu sein schien, jedoch ,zwischen den Zeilen' deutlich wurde. Auch Personen wurden in ihrer Vorbildfunktion erkennbar, ohne dass sie als direkte Vorbilder angesehen wurden.

Die Fragestellung der ELSAS-Studie war problembezogen (vgl. Brüsemeister 2000). Hier wurde das Wort ,Vorbild' verwendet. Der Erkenntnisgewinn war methodisch begrenzt. Limitationen bestanden durch die Beschaffenheit des Datenmaterials. Kurze Antworten, die häufig Aufzählungen von Eigenschaften beinhalteten, erschwerten z.B. eine weitere Zusammenfassung. Zudem steht die Inhaltsanalyse nach Mayring als zusammenfassendes und Kategorien generierendes Verfahren in der Kritik, selten auftretende Phänomene nicht zu berücksichtigen, da Kategorien durch Bünde- 
Diskussion

lung und Zusammenfassung ähnlicher Passagen nur häufiger erwähnte Phänomene einbeziehen.

Eine Gefahr bei der Auswertung des ersten Teils der Studie bestand darin, einen reproduzierten Vorbildbegriff wiederzugeben. Im Vorhinein bestehende Begriffsdefinitionen könnten wie eine Schablone wirken, die die Studienteilnehmer einschränkt, sodass nur das wiedergegeben wird, was im allgemeinen Sprachgebrauch unter einem Vorbild verstanden wird. Latente Inhalte möglicher Vorbildwirkungen gehen dabei jedoch verloren. Diese Erkenntnisse wurden für die Durchführung der Interviews berücksichtigt, bei denen bewusst der Begriff ,Vorbild‘ vermieden wurde.

So gelang es durch den zweiten Studienteil, sich der Thematik ,Vorbilder' indirekt und ohne Denkschablone zu nähern. Die Interviewpartner konnten frei entscheiden, über welche Personen sie berichten wollten, und auch Akteure mit einschließen, die eine Vorbildwirkung hatten, aber nicht unbedingt der Definition von Vorbildern der Studierenden entsprachen oder als solche wahrgenommen wurden. Das konnten Freunde sein, die zum Teil Vorbildfunktionen erfültten, jedoch bei der direkten Frage nach Vorbildern nicht genannt wurden. Auf die Nennung des Begriff ,Vorbild“ in der Erzählaufforderung wurde auch deshalb verzichtet, um die Interviewpartner nicht mit der impliziten Annahme unter Druck zu setzen, jeder müsse Vorbilder haben.

Vorteile Narrativer Interviews sind, dass die Interviewten in ihrer eigenen Sprache antworten können und die Aussagen nicht durch Vorformulierungen eingeschränkt sind. Typischerweise werden Aspekte geschildert, die subjektiv von Bedeutung sind. Beispielhaft ist die umfangreiche Kritik an negativen Erlebnissen im Studium. Dies kann zum Teil methodisch erklärt und u.a. auf die Fragestellung zurückgeführt werden. Die Einstiegsfrage überließ es den Probanden auch zu entscheiden, wer für sie „Von Bedeutung war“ und ob dies im positiven oder negativen Sinn zu verstehen war. Neben der Tatsache, dass die Interviewerin selbst Studierende der Medizin war, waren die per Inhaltsanalyse gewonnenen Ergebnisse der ELSAS-Studie für eine Vorab-Sensibilisierung von Vorteil: ,Insider-Wissen' konnte genutzt werden, um die Perspektiven der Akteure und Akteurinnen besser zu verstehen bzw. zu rekonstruieren (Meyer et al. 2012). Nachteilig war eine Tendenz zur ,sozialen Erwünschtheit', insoweit Antworten manchmal so ausfielen, dass sie auf Zustimmung der Interviewerin treffen sollten. Eine Einflussnahme während der Interviews sollte durch geeignetes Training vermieden, kann jedoch nicht vollständig ausgeschlossen werden (Richards 
Diskussion

und Emslie 2000). Bei der Auswertung könnten auch Vorannahmen, die auf eigenen Erfahrungen als Medizinstudentin beruhten, die Interpretation und Analyse beeinflusst haben. Um diese Einflüsse und damit den Verlust relevanter Deutungsmöglichkeiten gering zu halten, wurden analytische Fragen regelmäßig in der Arbeitsgruppe diskutiert, in einem "reiterativen Prozess" (Meyer et al. 2012) im Austausch mit und unter Überprüfung durch andere (Einbettung in das Forschungskolloquium des Instituts für Allgemeinmedizin Göttingen, Teilnahme an einer externen Forschungswerkstatt der Palliativmedizin der UMG).

\subsection{Schlussfolgerungen und Implikationen für die Praxis}

Unsere Studie zeigt die große Bandbreite möglicher Erfahrungen mit Vorbildern im Studium. Viele Erwartungen der Studierenden an vorbildliches ärztliches Verhalten werden von den beobachteten Ärzten nicht erfüllt. Eine bedeutsame Rolle spielen vielmehr Kommilitonen, die nicht nur als Unterstützer bei der Bewältigung des Studiums, sondern auch als Vorbilder wirken. Sie übermitteln wichtige inoffizielle Lerninhalte und helfen, in die Rolle als Medizinstudent/in hineinzufinden.

Im Verlauf des Studiums verändert sich durch den Zugewinn neuer Erfahrungen die Haltung der Studierenden ihren Vorbildern gegenüber. Die Beziehung zum Vorbild ist prozesshaft, ihre Wirkung und Funktion häufig unbewusst und entzieht sich teilweise dem Einfluss von Dozenten.

Eine Wirkung von Vorbildern und prägenden Personen auf Studierende ist deutlich erkennbar. Vorbilder erfüllen nicht nur ,klassische', sondern auch ,unkonventionelle Funktionen. Studentische Vorbilder dienten beispielsweise als 'Strategie zum Austausch von Wissen'. Das Lernen am Vorbild scheint einem Wandel zu unterliegen und sich auch auf andere Formen wie kollaborierendes Lernen unter Kommilitonen zu erstrecken.

Vielen Akteuren (insbesondere dem Krankenhauspersonal) scheint nicht bewusst zu sein, dass sie Vorbildfunktionen haben und damit zur beruflichen Sozialisation von Studierenden beitragen. Gerade unbewusstes, vorbildhaftes Verhalten von Dozenten könnte im Sinne einer indirekten Vorbildfunktion große, bisher unterschätzte Wirkung auf die Entwicklung zur Ausprägung der ärztlichen Persönlichkeit von Studierenden nehmen. Dass Vorbilder Interaktionsmuster vorgeben und auch das zukünftige Verhalten der Studierenden Patienten gegenüber beeinflussen können, wird von unserer 
Diskussion

Studie bestätigt. Sie nehmen Einfluss darauf, wie Medizinstudierende zukünftiger Generationen sich selbst und anderen gegenüber verhalten. Dabei geht es nicht nur um ihr Verhalten Patienten gegenüber, sondern auch um ihr Verhalten als zukünftige Lehrende gegenüber Studierenden und Kollegen.

Die Beziehung zum Vorbild ist ,prozesshaft': Zu Beginn des Studiums schienen die Begegnungen mit Vorbildern von mitgebrachten Erwartungen bestimmt zu sein. Sie wurden teilweise enttäuscht, z.T. verändert. In diesem Prozess veränderte sich die Haltung der Studierenden gegenüber potentiellen Vorbildern. Bei der nächsten Begegnung mit demselben Dozenten mochten die Erwartungen von Studierenden an inn als mögliches Vorbild und die Bereitschaft, von ihm/ihr zu lernen, bereits ganz andere sein.

Ein Verständnis der Umstände, unter denen Medizinstudierende ausgebildet werden, und der Möglichkeiten, die das Lernen am Vorbild beinhaltet, kann zur Verbesserung der Ausbildung genutzt werden. Ereignisse im Studium zu reflektieren, das Verhalten von Dozenten zu hinterfragen und einen kritischen Umgang mit vorgelebten ärztlichen Verhalten zu lernen, wird im Studium derzeit nicht aktiv gefördert. Die Fähigkeit zur Reflexion könnten Studierende exemplarisch von Vorbildern lernen. Lernen am Vorbild sei „eine Form des Wissenserwerbs“, die sinnvoll und für viele hilfreich sei, jedoch nicht voll ausgeschöpft werde. Es spräche „einiges dafür, den Prozess des Lernens zum einen früher zu beginnen und zum anderen zu systematisieren", schrieben Terzioglu et al. 2003 im Deutschen Ärzteblatt.

Bei der Gestaltung der Lehre an medizinischen Fakultäten könnte die Erweiterung um das Lernen von Vorbildern in Konzepte einer lern-fördernden Umgebung integriert werden und bei der Entwicklung von Schlüsselkompetenzen helfen. Vorbilder tragen als Teil des ,hidden curriculum zum Lernen bei und vermitteln unausgesprochene Verhaltensmaßstäbe, jedoch wären sie auch bewusst einsetzbar. Wie die wachsende Zahl von Mentoring-Programmen an Hochschulen zeigt, lassen sich Wissens- und Erfahrungsvorsprung erfahrener Mitglieder der Ärztegemeinschaft nutzen, um den Studierenden den Einstieg in die Profession zu erleichtern.

Die Ergebnisse dieser Studien lenken den Blick auf ein Problemfeld: Negative Erfahrungen während des Medizinstudiums werden überwiegend während klinischer Praktika gemacht (Baldwin et al. 1988; Witte 2010; Gágyor et al. 2012). Dies könnte ,Erfahrungslernen' verhindern oder in die falsche Richtung lenken. Durch die o.g. Er- 
Diskussion

gänzung des Curriculums könnten Vorbilder, die den Studierenden Lust auf den Beruf machen, ideales ärztliches Verhalten demonstrieren und damit Ideale der Profession vermitteln, zum Entwickeln eigener Handlungskompetenzen beitragen. Unter den gegebenen Bedingungen müssen Studierende derzeit selbstständig nach Strategien suchen, die innen ihr ,Überleben“ im medizinischen Umfeld sichern. Sich einer Medizinkultur anpassen zu müssen, die Studierende und ihren Lernprozess nicht fördert, sondern Leistungs- und Selektionsdruck ausübt, verstellt den Blick auf positives Vorbildverhalten und negiert ggf. sogar den Einfluss von Vorbildern. Wichtig wäre, Studierende in ihrer Rolle als Lernende anzuerkennen und zu bestätigen. Erst dann können sie ihrem Wunsch, gute Ärzte zu werden, nachkommen und sich in produktiver Art mit ihrer zukünftigen Rolle und über Vorbilder vermittelte Inhalte auseinandersetzen.

Vorbilder spiegeln ein Stück ,soziale Wirklichkeit' wider. Es Studierenden selbst zu überlassen, sich zu sozialisieren und ein Verständnis von Professionalität zu erarbeiten, ist kritisch zu betrachten. Und Kommilitonen mögen gute Vorbilder sein, aber auf ihre Haltungen, ihre Vermittlung von Verhaltensweisen kann eine Fakultät wenig Einfluss nehmen. Wenn medizinische Fakultäten auf die implizite Vermittlung professioneller Verhaltensweisen vertrauen, sind zumindest die Inhalte zu bedenken.

Dass sich Vorbildfunktionen nicht allein auf das Verhalten von Lehrkräften beschränken, ist jedenfalls deutlich geworden. Auch eine ,Medizinkultur' vermittelt ein Bild einer Profession und hat Vorbildfunktionen. Ob eine Berufsgruppe in ihren Kulturraum (z.B. der medizinischen Fakultät) Fehler verzeiht, entscheidet schließlich darüber, ob Studierende sich in neuen Rollen ,erproben' können. Das allerseits erwünschte ,Erfahrungslernen' ist nur dann möglich, wenn die Studierenden in ihrer Entwicklung begleitet und gefördert werden. Erst dann können sie ihr eigenes Potenzial erkennen und zunehmend der hohen Erwartung entsprechen, die an diesen Berufsstand sowohl von ihren Lehrenden als auch von den späteren Patienten gestellt werden. Friedrichs et al. skizzieren eine Kultur der Anerkennung:

„Sie macht Wertschätzung deutlich, zeigt positive Zuwendung und schenkt Zeit; sie fördert die Fähigkeit zum Perspektivenwechsel, lebt eine stärkenorientierte Pädagogik und basiert auf einem respektvollen und höflichem Umgang miteinander. Darüber hinaus achtet und schützt sie Vielfalt, ermutigt Menschen und ist die Basis zur Entwicklung von Selbstvertrauen und Selbstwirksamkeit" (Friedrichs et al. 1997, S. 315). 
Diskussion

In Bezug auf die Vorbildwirkung erscheint die Forderung von Omer und Schlippe (2010) interessant, die Distanz einer traditionellen Autorität in Präsenz umzuwandeln, bei der die „wachsame Sorge“ Hauptbestandteil ist. Dies bedeutet, den Lernprozess der Studierenden begleitend zu unterstützen, anstatt von außen Ideale und Verhaltensregeln zu setzen. Die zukünftige Rolle von Vorbildern könnte z.B. darin bestehen, dafür zu sorgen, dass Studierende bei der Verarbeitung prägender Erfahrungen und darin unterstützt werden, eigenständig zu lernen (engl. „to guide experiences“). Böhle schrieb zur Vermittlung von Schlüsselqualifikationen:

„Man kann sie nicht ,lehren“ wie fachliches Wissen oder fachliches Können (...) vielmehr wurde bewusst, dass die Lernenden Schlüsselkompetenzen bzw. Handlungskompetenzen dann ausbilden, wenn sie in Handlungssituationen kommen, in denen genau diese Schlüsselqualifikationen gebraucht werden. Damit wurde die Berufsausbildung offen für das Handlungslernen." (Böhle et al. 2008)

Dozenten hätten dann weniger die Aufgabe eines unmittelbaren Erfahrungs- und Wissenstransfers (wie es bei Kommilitonen beobachtet wurde), sondern die Funktion von ,Begleitern', die den Studierenden das Erleben eigener positiver Erfahrungen ermöglichen. Der Erfolg sozialisatorischer Interaktionen an der Universität wäre nicht länger an eine möglichst optimale didaktische Anleitung durch Hochschullehrer gebunden, sondern an die Möglichkeit der studentischen Teilnahme an prägenden Erlebnissen, die neue Handlungskompetenzen eröffnen (vgl. Böhle et al. 2008). Wie eine solche Unterstützung konkret aussehen kann, ist eine Aufgabe zukünftiger Forschung. 


\section{Zusammenfassung}

Hintergrund: Vorbilder im Medizinstudium tragen als Teil des, hidden curriculum (heimlichen Lehrplans) zu impliziten Lernprozessen von Studierenden bei und vermitteln unausgesprochene Verhaltensmaßstäbe. Bisherige Studien konzentrieren sich vor allem auf die Vorbildwirkung von Ärzten auf Medizinstudierende. Ziel dieser Studie war, in einem offenen Design die Bedeutung und Wirkung verschiedener Personen für die eigene berufliche Orientierung, Identifikation und Arztwerdung aus der Perspektive der Studierenden beschreiben zu lassen.

Fragestellung: Welche Personen sind Medizinstudierenden im Rahmen ihres Studiums wichtig? Was vermitteln prägende Personen und Vorbilder, und welche Funktionen erfüllen sie? In welchem Kontext begegnen Studierende ihren Vorbildern?

Methode: Zunächst wurde das im Rahmen einer Online-Befragung von Medizinstudierenden über Erfahrungen mit Vorbildern erhobene Textmaterial mittels Qualitativer Inhaltsanalyse nach Mayring ausgewertet. Dann wurden 15 Studierende in narrativen Interviews befragt und ihre Erfahrungen mit Vorbildern nach Prinzipien der Grounded Theory ausgewertet.

Ergebnisse: Vorbildfunktionen übernahmen nicht nur Personen, die bewusst und typischerweise als Vorbilder wahrgenommen werden, sondern auch Menschen im engeren Umfeld, insbesondere Kommilitonen, die wichtige inoffizielle Lerninhalte übermittelten und halfen, in die Rolle als Medizinstudent/in hineinzufinden. Beim Erlernen ärztlichen Verhaltens fühlten sich viele Studierende allein gelassen und suchten oft selbstständig nach Strategien zur Entwicklung einer professionellen Identität. Sie berichteten von einer Diskrepanz zwischen eigenen Idealvorstellungen und real erlebtem Verhalten von Dozenten, die sich ihrer Vorbildwirkung häufig nicht bewusst waren. Dabei ging es nicht nur um professionelle Verhaltensweisen im Kontakt mit Patienten. Studierende vermissten gerade auch Akzeptanz und Wertschätzung seitens der Lehrenden.

Schlussfolgerungen: Vorbilder nehmen Einfluss darauf, wie sich angehende Ärztinnen und Ärzte in ihrem späteren Beruf verhalten, und das beginnt bereits während des Studiums. Bei der Gestaltung der Lehre an medizinischen Fakultäten könnte das Lernen von Vorbildern bewusst in Lehrkonzepte integriert werden und z.B. bei der 
Zusammenfassung

Entwicklung von Schlüsselkompetenzen eingesetzt werden. So ließe sich eine die Entwicklung der Studierenden unterstützende ,Medizinkultur's schaffen. 


\section{$8 \quad$ Anhang}

\subsection{Information und Aufklärung für Studienteilnehmer/innen der Interviews}

Georg-August-Universität Göttingen

Abteilung für Allgemeinmedizin,

Robert-Koch-Str. 40, 37075 Göttingen

Abteilungsdirektorin:

Prof. Dr. Eva Hummers-Pradier

Wissenschaftliche Studienleitung:

Prof. Dr. Wolfgang Himmel

Dr. med. Ildikó Gágyor,

Information und Aufklärung für Studienteilnehmer/innen zur Teilnahme am Interview

Liebe Studentin,

Lieber Student,

im Verlauf des Medizinstudiums begegnen Studierende vielen Menschen, die einen Einfluss auf ihre Entwicklung als Mensch, wie auch als zukünftige Ärztinnen und Ärzte haben.

Im Rahmen dieser Studie möchten wir untersuchen, welche Erfahrungen Medizinstudierende in Begegnungen mit Menschen außerhalb des regulären Lehrplans machen.

Wir möchten Sie einladen, an einem Interview teilzunehmen, in dem Sie die Gelegenheit erhalten, einzelne Situationen, die in Ihrem Studium für Sie von Bedeutung waren, aus ihrer Perspektive zu schildern. Die Teilnahme an dieser Studie ist freiwillig, eine Nichtteilnahme bringt keinerlei Nachteile mit sich. Das Interview wird min- 
Anhang

destens 30 Minuten in Anspruch nehmen. Das Gespräch kann jederzeit abgebrochen oder zu einem späteren Zeitpunkt (z.B. am nächsten Tag) fortgesetzt werden. Im Anschluss an das Interview möchten wir Sie bitten, einen begleitenden Kurzfragebogen mit Daten zu Ihrer Person auszufüllen.

Das Interview wird auf Tonband aufgenommen und anschließend wörtlich niedergeschrieben. Die Aufnahmen dienen lediglich dem hier beschriebenen Zweck; nach Beendigung der Studie werden sie zwar archiviert, aber nicht für weitere Studien oder im Unterricht für die Studierenden verwendet. Die Auswertung des Interviews sowie des Kurzfragebogens erfolgt pseudonymisiert, d.h. ohne Verwendung Ihres Namens oder Ihrer persönlichen Daten; Rückschlüsse auf Ihre Person werden daher nicht möglich sein.

Sollten Sie noch Fragen haben, rufen Sie mich bitte an.

Dr. med. Ildikó Gágyor (wissenschaftliche Leitung)

Abteilung Allgemeinmedizin

UMG Göttingen

T: $0551 / 3914226$

Email: igagyor@gwdg.de

Jenny Bücken, Medizinstudentin

Tel. 0551/2713195 
Georg-August-Universität Göttingen

Abteilung Allgemeinmedizin,

Robert-Koch-Str. 40, 37075 Göttingen

Abteilungsdirektorin:

Prof. Dr. Eva Hummers-Pradier

Wissenschaftliche Studienleitung:

Prof. Dr. Wolfgang Himmel

Dr. med. Ildikó Gágyor

\section{Einverständniserklärung}

Ich,

wurde voll-

ständig über die Durchführung des Interviews und die damit verbundenen Rahmenbedingungen aufgeklärt. Ich hatte die Möglichkeit Fragen zu stellen und habe die Antworten verstanden und akzeptiere sie.

Ich hatte ausreichend Zeit, mich zur Teilnahme an dem Interview zu entscheiden und weiß, dass meine Teilnahme freiwillig und mit keinerlei persönlichem Nutzen oder mit Nachteilen für mich verbunden ist. Ich weiß, dass ich jederzeit und ohne Angabe von Gründen diese Zustimmung widerrufen kann, ohne dass sich dieser Entschluss nachteilig für mich auswirken wird. Die bisher erhobenen Daten würden in diesem Fall vernichtet werden. Ich bin damit einverstanden, dass die im Rahmen der Studie erhobenen Daten in pseudonymisierter Form unter den Bedingungen des Datenschutzes gespeichert und zu Forschungszwecken verwendet werden. Die personenbezogenen Daten werden nicht an Dritte weitergegeben. 
Ich bin damit einverstanden, dass das Gespräch auf Tonband aufgezeichnet wird und erkläre hiermit meine freiwillige Teilnahme an dem Interview.

Ich habe die Informationen zur Studie und eine Kopie der Einverständniserklärung erhalten.

\subsection{Liste der Hauptkategorien der ELSAS-Studie mit zugehörigen Unterkategorien}

1. Ärzte, die in herausfordernden Berufssituationen professionell handeln

- Ärzte, die Zeit und Empathie für Gespräche aufbringen, die für Patienten bedeutsam sind

- Ärzte, die in herausfordernden Situationen Ruhe bewahren

- Ärzte, die sich trotz schwieriger Umstände Zeit für Patienten nehmen und freundlich bleiben

- Ärzte mit ganzheitlicher Arbeitsweise

- Ärzte, die erfolgreich unterschiedliche berufliche Anforderungen erfüllen und über ein effektives Zeitmanagement verfügen

2. Ärzte mit großem Fachwissen und Kompetenz bei praktischen Tätigkeiten 
Anhang

3. Ärzte, die fachlich kompetent sind und zugleich als Personen besondere Eigenschaften haben

- Ranghohe Ärztin, die Bescheidenheit, Freundlichkeit, Höflichkeit, Geduld und Herzlichkeit zeigt

- Ranghoher Arzt, der Ruhe, Höflichkeit und fachliche Professionalität besitzt

- Ranghoher Arzt, der professionell über Krankheiten spricht und einen positivem Einfluss auf Studierende hat

4. Ärzte, die selbstbewusst und kompetent auftreten

- Ranghoher Arzt, der eine persönliche Gewohnheit mit den Studierenden teilt

- Arzt, der bei klinischer Tätigkeit selbstsicher wirkt und offen im Meinungsaustausch mit Studierenden ist

- Erfahrene Ärzte, die Ruhe und Abgeklärtheit bei klinischer Tätigkeit zeigen

- Arzt in Lehrveranstaltung, der Ruhe und Souveränität ausstrahlt

- Ranghohe Ärztinnen, die in Lehrveranstaltungen kompetent und taff wirken

- Ranghoher Arzt, der bei klinischen Tätigkeiten ruhig, kompetent und gewissenhaft auftritt

- Arzt, der bodenständig und kompetent wirkt

5. Ärzte mit sozialen Kompetenzen im Umgang mit Patienten

- Ärzte, die geschickt mit schwierigen Patienten umgehen

- Ärzte, die respektvoll und rücksichtsvoll mit Patienten umgehen

- Ärzte mit Feingefühl im Umgang mit Patienten und Angehörigen

- Ärzte mit Kompetenz im Umgang mit sterbenden Patienten

6. Pflegekräfte mit Bereitschaft, unter schwierigen Arbeitsbedingungen gute Arbeit zu leisten

7. Ärzte, die einen hohen Anspruch an den Beruf vermitteln 
- Ärzte, die für ihre Patienten Verantwortung übernehmen und sich für ihr Wohlergehen einsetzen

- Ärzte, die Gewissenhaftigkeit und wissenschaftlichen Anspruch an den Beruf zeigen

- Lehrende Ärzte, die eine kritisch hinterfragende Haltung zu Entwicklungen an medizinischen Hochschulen einnehmen

8. Ärzte, die Studierende wertschätzen und unterstützen

- Ärzte, die einen wertschätzenden und nicht hierarchischen Umgang mit Studierenden während Lehrveranstaltungen zeigen

- Ranghoher Arzt, der die Ausbildung von Studierenden ernst nimmt und sie fördert

- Lehrende Ärzte, die sich bemühen, Studierenden das Lernen zu erleichtern und sie unterstützen

- Ärzte, die sich Studierenden gegenüber gerecht und freundlich verhalten

- Ärzte, die in Lehrveranstaltungen kollegial mit Studierenden umgehen

- Ärzte, die Wert auf die Meinung der Studierenden legen

- Ärzte, die Studierende nicht als Last, sondern als Hilfe sehen und ihnen das Gefühl vermitteln, nützlich zu sein

- Lehrende Ärzte, die Studierende in klinische Tätigkeiten einbeziehen, auf den einzelnen Studenten eingehen und ihre Selbstständigkeit fördern

- Ärzte, die Studierende in Lehrveranstaltungen gut betreuen und Wert auf die Ausbildung jedes einzelnen Studierenden legen

- Betreuerin der Doktorarbeit, die großes Engagement und gerechtes Verhalten Studierenden gegenüber zeigt

- Arzt, der das Selbstbewusstsein der Studierenden stärkt

9. Ärzte, die die Ausbildung der Studierenden ernst nehmen 
- Lehrende Ärzte, die das Anliegen verfolgen, Studierende über die eigene Lehrveranstaltung hinaus für den Beruf auszubilden

- Ärzte, die im klinischen Alltag Zeit für die Lehre aufbringen

- Lehrender Arzt, der Wert auf die Vermittlung sozialer Kompetenzen legt und deren Relevanz verdeutlicht

- Ranghoher Arzt, der gute Lehre macht und sich bemüht, neben theoretischen auch soziale Lerninhalte zu vermitteln

10. Ärzte, die in Lehrsituationen Studierende für den Beruf begeistern

- Ärzte, die Studierende durch ihr Engagement und die Gestaltung von Lehrveranstaltungen motivieren

- Lehrende Ärzte, die sich bemühen, in Lehrveranstaltungen Interesse bei Studierenden für ihre Arbeit zu erwecken und Wert auf nachhaltige Lehre legen

- Ärzte, die die Studierenden durch ihre soziale und fachliche Kompetenz für den ärztlichen Beruf motivieren

- Lehrende Ärzte, die Freude an der Lehre und am Beruf vermitteln und damit Studierende anstecken und begeistern

- Ärzte, die Begeisterung und Liebe zum Beruf vermitteln

- Ärzte, die eine freundliche Ausstrahlung besitzen und Engagement und Humor in Lehrveranstaltungen zeigen

- Lehrende Ärzte mit Begabung, Studierenden Wissen in Lehrveranstaltungen mit Erfolg zu vermitteln

- Lehrender Arzt, der anhand von realen Beispielen die Relevanz von Lerninhalten verdeutlicht

11. Ärzte, die Führungskompetenz und Kollegialität vereinen

- Ranghoher Arzt, der trotz Autorität ein gutes Verhältnis zu Mitarbeitern besitzt

- Ranghoher Arzt, der aufgrund von Autorität, Wissen und seinem Umgang mit Patienten Vorbild ist 
- Kompetente ranghohe Ärzte, die respektvollen Umgang mit rangniedrigeren Mitarbeitern und Interesse an ihrer Ausbildung zeigen

- Ranghoher Arzt, der trotz hoher Arbeitsbelastung einen menschlichen Führungsstil zeigt

- Ranghohe Ärzte, die Kompetenzen in den Bereichen Kommunikation, Mitarbeitermotivation und Krisenmanagement besitzen

- Ärzte, die auf Mitarbeiter eingehen und Wert auf Zusammenarbeit legen

- Ärzte, die einen kollegialen Umgang unter Mitarbeitern pflegen und sich nicht hierarchisch verhalten

- Ärzte, die trotz herausfordernden praktischen Tätigkeiten einen freundlichen Umgang mit Mitarbeitern bewahren

12. Ethische Grundprinzipien medizinischen Handelns implizit vermitteln

- Ranghoher Arzt, der am eigenen Beispiel Gleichberechtigung aller Menschen demonstriert

- Ärzte, die eine patientenzentrierte und humane Einstellung zum Beruf vermitteln

- Ranghoher Arzt, der am eigenen Beispiel zeigt, dass eine ehrliche Kommunikation mit Angehörigen schwer kranker Patienten möglich ist

- Pflegekraft, die einen Patientenumgang mit Würde und Menschlichkeit demonstriert

- Ärzte, die einen respektvollen und verständnisvollen Patientenumgang zeigen und die Einstellung, Patienten und ihr Schicksal wichtig zu nehmen vermitteln

- Ärzte, die eine Ausrichtung der Behandlung am Wohl des Patienten vermitteln

- Ärzte, die Unsicherheit, Fehler und die Begrenztheit des eigenen Wissens eingestehen

13. Ärzte, die in der medizinischen Hierarchie oben stehen 
- Ärzte eines bestimmten hohen akademischen Grades

- Bestimmte [namentlich genannte] Ärzte hohen Ranges

- Ärzten mit einem Wissensvorsprung gegenüber Studierenden

14. Ärzte, die trotz ihres hohen Ranges Interesse an Studierenden haben und durch ihre Persönlichkeit Vorbilder sind

- Ranghoher Arzt, der persönliches Engagement für die Lehre zeigt

- Ranghohe Ärzte, die Studentennähe zeigen und Mensch geblieben sind

- Ranghoher Arzt, der trotz hoher Leistung und Kompetenz Mensch geblieben ist

- Ranghoher Arzt, der einen natürlichen und freundlichen Umgang mit Studierenden zeigt und Zufriedenheit und Gelassenheit im Beruf und Privatleben ausstrahlt

- Ranghoher Arzt, der persönliches Engagement für Studierende zeigt

- Ranghohe Ärzte, die Bereitschaft zur Weitergabe ihres großen Wissens zeigen

15. Kommilitonen, die engagiert, kompetent und unterstützend sind

- Ältere Studierende, die ihre Erfahrung bereitwillig mit Jüngeren teilen

- Studentische Hilfskräfte mit großem Wissen in Lehrveranstaltungen

- Auftreten und Wissen studentischer Hilfskräfte in der Lehre

- Hilfsbereitschaft und Kompetenz eines Studenten im Praktischen Jahr

- Effektive Lernstrategien von Kommilitonen

- Kommilitonen mit Fleiß und Hilfsbereitschaft anderen Studierenden gegenüber

- Engagement Studierender mit studentischem Amt 
- Charakter einer Kommilitonin und ihr Verhalten in der direkten Interaktion

- Motivation und engagierte Einstellung von Kommilitonen

\subsection{Beispiel-Memo: Phänomen ,Distanz“}

Die Distanz, von der Studierende in Bezug auf ihr Verhältnis zu Professoren bzw. Lehrenden berichten, ist bereits beschrieben worden. Sie steht bisher in einem Zusammenhang, in dem sie als ursächliche Bedingung dafür erscheint, dass Studierende zum einen wenig Kontakt zu Angehörigen dieser Personengruppen haben, zum anderen aber auch als ursächliche Bedingung dafür, dass Studierende andere Strategien entwickeln. Sie können sich z.B. vorbildliche Teilaspekte von unterschiedlichen Personen zusammensuchen oder nach anderen Personen suchen, mit denen sie leichter in Kontakt kommen und an denen sie sich orientieren können.

Das Phänomen Distanz zu Professoren und Lehrenden bringt die Studierenden aber auch dazu, nach anderen Möglichkeiten zu suchen „das Soziale ins Studium zu holen“, wie es S07 beschreibt. Dass ein Bedarf an Orientierung an Vorbildern gerade in Bezug auf die ersten Erfahrungen im Krankenhaus besteht, zeigen die folgenden Beispiele: In beiden Situationen werden die Studentinnen mit den Patienten allein gelassen.

Welchen entscheidenden Einfluss erste Erfahrungen mit Patienten für Studierende haben, zeigt sich auch bei S13. Dessen erste Patientenkontakte werden, im Gegensatz zu den beiden Genannten, betreut und tragen durch das Einbezogen werden durch den betreuenden Arzt dazu bei, dass der Student überhaupt Interesse an Patientenkontakt entwickelt:

„alles andere war für mich irgendwie vorher ausgeschlossen und dann war es aber so was war denn da der Auslöser also richtig war das erst dass ich auf was anderes gekommen bin auf den Trichter dass ich durch ähm X-(Name eines Arztes) der ist Assistenzarzt in der X(Name einer Abteilung)." (S13 003)

Die Distanz zu ihren Lehrenden wird aber auch noch an anderer Stelle zu einem Problem für die Studierenden: Zum einen trägt sie dazu bei, dass keine Vertrauensbasis aufgebaut wird. Dies verhindert eine entspannte Lernatmosphäre, in der offen Fragen gestellt werden können. 
Anhang

„....ähm ich fand das sehr angenehm dass er halt auch nicht die ganze Zeit am Tisch //l:mhm// sondern wir auch hauptsächlich mit den mit den mit den mit den Hiwis warn //I:mhm// und da wars ja auch in Ordnung //l:mhm// da konnte man sowieso alles fragen also die warn locker und es war auf einer Ebene mehr und er war dann schon irgendwie der .. der Prof irgendwie //l:mhm// aber ähm er war dann auch nicht so häufig da so dass man irgendwie das Gefühl hatte man konnte da so Fragen stellen." (S12 19)

Zum anderen sehen die Studierenden jedoch auch einen Bedarf, Medizin als Fach, bei der die Arbeit mit Menschen im Mittelpunkt steht, von Menschen und nicht nur aus Büchern zu lernen. Für sie stellt sich die Frage, durch wen „das Soziale ins Studium" gebracht wird, wenn nicht durch Lehrende? Unabhängig von Zielen für den späteren Beruf scheinen diese Aufgabe zunächst Kommilitonen und andere Bezugsund Vertrauenspersonen zu übernehmen. Sozialer Rückhalt wird von vielen Studierenden als wichtig für sie und ihr Studium beschrieben, erleichtert die Bewältigung des Studiums. Jedoch stellt sich in Bezug auf das Forschungsinteresse dieser Arbeit die Frage, ob diese Gruppe eine Vorbildfunktion für die Studierende übernimmt.

In diesem Zusammenhang muss Distanz also auch als Kriterium für die Definition der Studierenden von Vorbildern betrachtet werden. Studierende scheinen Vorbilder als Personen zu sehen, die „schon etwas erreicht haben, was sie noch zu erreichen wünschen“. Dazu gehört eine Überlegenheit des Vorbilds auf dem angestrebten Gebiet:

„und von ihm hab ich unheimlich viel in der Richtung gelernt auch was Forschung angeht was auch ähm Unipolitik angeht sag ich mal." (S08 09)

Zu dem Vorbild wird aufgeschaut, was einen gewissen Abstand zu der Situation der Studierenden vorauszusetzen scheint. Als ein Kriterium könnte hier also das Alter der Studierenden herangezogen werden. Dies bestätigt sich auch in dem folgenden Zitat:

„ähm von meiner von mein Leuten von der WG die würd ich schon viele als meine Vorbilder bezeichnen //l:hmmhm/ einfach weil sie älter sind so und weil ich den so ich die kenne //l:hm// $\mathrm{hm}$ Professoren das ist dann halt auch irgendwie wieder relativ weit weg so man weiß ja nicht kommt man da irgendwo mal hin." (S07 55)

Gleichzeitig wird an dieser Stelle ein weiteres Kriterium angeführt, das Kriterium der Erreichbarkeit. Von dem Studenten wird überlegt, ob ein Professor für inn überhaupt ein Vorbild ist, zumal er sich zum einen nicht sicher ist, ob er diese Position überhaupt erreichen kann und zum anderen diese vielleicht auch gar nicht erreichen will. 
Anders verhält es sich bei S08, der zwar sicher ist, dass er die gleichen Ziele wie sein Vorbild verfolgt, es aber gerade als vorbildlich ansieht, dass er diese aufgrund der hohen Qualität und Leistungsstärke des Vorbilds vielleicht nie erreichen wird:

„P: Also der X-(Name eines Freundes5) ist sicherlich ein Vorbild für mich //I:mhm// der ist vielleicht sogar ja .. wie soll ich sagen der ist vielleicht so an der Stelle wo der so oder so ... weiß ich nicht so gut einfach dass ich denke da will ich vielleicht gar nicht ganz drankommen //l:mhm// das was er tut //l: okay// aber deswegen vielleicht grade deswegen ist es auch Vorbild weil ich denke da kann ich zumindest ähm mich ranarbeiten //l:mhm// grad was Forschung angeht sozusagen oder wie auch sozusagen man so $\mathrm{n}$ Weg gehen kann //l:mhm// innerhalb von Klinik und Forschung wann man welche Schritte machen muss .. das ist ganz sicher ein Vorbild für mich //l: ja// .. und also vielleicht ist ja doch ist das ein wichtiges Vorbild //l:mhm// weil das .. auch vielleicht noch so nah ist sozusagen schon dass ich schon noch verstehe was passiert //l:mhm// das ich das nachempfinden kann vielleicht nicht jeden nicht alles schaffe nachzumachen sozusagen //l: ja// aber .. schon mir da viel ähm dran lernen konnte." (S08 84)

Aus den Worten von S08 spricht die Bewunderung und Hochachtung des Vorbilds was der Eigenschaft eines Vorbildes entspricht, als Ideal einer bestimmten Funktion angesehen zu werden. Vorbilder haben Eigenschaften, die für die Studierenden schwer erreichbar erscheinen und auf einem Leistungsniveau liegen, das auch nur von wenigen erreicht wird. Auch in ihrem gesellschaftlichen Ansehen werden Ärzten gerne als "Götter in Weiß" bezeichnet. Fähigkeiten, wie das Leben von Patienten akut zu retten, werden auch von den Studierenden an ihren Vorbildern bewundert. Es geht hier wieder um ein Ideal, von dem sie selbst noch weit entfernt sind. Die Frage ist, ob auch Menschen, die in einer ähnlichen Position sind wie sie selbst Vorbildfunktionen übernehmen können: S01 bezeichnet einen ihrer Kommilitonen, den sie als Studenten mit vorbildlichen Eigenschaften wie Fleiß und Engagement beschreibt, als „Leitsymbol“ (S01 16). Und auch S14 beschreibt eine ihrer Kommilitoninnen folgendermaßen:

„also X-(Name der Freundin) ist so nen Mensch wo jeder so denkt krass wie kriegt sie das alles unter einen Hut //l: mhm// also sie ist halt ähm.. was ich halt an Menschen sehr sehr schätze und ganz arg wichtig finde sie kann sehr gut zuhören //l: mhm// und ähm.. geht äh dann.. auch auf das Gesagte ein also man kann sich sehr gut mit ihr unterhalten //l: $\mathrm{mhm} / /$ und ähm das ist so ne Qualität finde ich die man eigentlich nur bei wenigen Leuten so ausgeprägt findet wie bei ihr also sie kann sich sehr gut in andere Leute reinversetzen." (S14 143) 


\subsection{Zitate des Ergebnisteils im Volltext}

„ich finde das ist generell ein Thema für //l:mhm// junge Ärzte irgendwie weil du ja eben jung bist und auch total unerfahren //I:mhm// und ähm .. ich als Patient würde vielleicht auch manchmal denken pff muss das jetzt sein habt ihr nicht noch jemanden der das schon ein bisschen länger macht und ähm vielleicht muss man dann auch manchmal einfach ähm .. ja Brust raus und dann durch die Situation und dann //l:mhm// so ja und auch eine Kompetenz ausstrahlen die noch nicht so gefestigt ist" (S11 119)

„genau das war das Wahlfach Endokrinologie //l:mhm// und sie hat .. sie hat so Geschichten erzählt wie sie zu ähm Damenbart steht ((lachen beide)) oder was sie zu ähm ach irgendwelchen .. äh.. welche zu irgendwelchen Pillen und Verhütungsmitteln“ (S05 103)

„ähm wie man dieses so man hat's vielleicht so ein bisschen gelernt wie man mit so unangenehmen Situationen umgeht //I:mhm// also wenn der Patient anfängt zu weinen oder //I:mhm// wie geh ich damit um wenn er auf einmal hibbelig wird oder so //l:ja// und .. das hat einem zumindest so für für später glaub ich ein bisschen Sicherheit gegeben weil ich jetzt zumindest besser weiß wie ich mit solchen Situationen umgehen kann." (S05 131)

„und ich finde dass sich das häufig auch son bisschen verbindet dass so zweckmäßiges Beisammensein aber man sich auch sympathisch findet es aber niemals ausreichen würde dass man jetzt zusammen in Urlaub fährt //l:mhm// irgendwie //l: ja ((lacht))// ja das ist etwas was ich eigentlich ja jetzt nicht mehr so möchte und //l:mhm// deswegen mich da auch son bisschen rausziehe hm //I:mhm// genau." (S12 15)

„ähm ich fand das sehr angenehm dass er halt auch nicht die ganze Zeit am Tisch //l:mhm// sondern wir auch hauptsächlich mit den mit den mit den Hiwis warn //I:mhm// und da wars ja auch in Ordnung //l:mhm// da konnte man sowieso alles fragen also die warn locker und es war auf einer Ebene mehr und er war dann schon irgendwie der .. der Prof irgendwie //l:mhm// aber ähm er war dann auch nicht so häufig da so dass man irgendwie das Gefühl hatte man konnte da so Fragen stellen“ (S12 19).

„man kann sich glaub ich schon auch auf ner sehr menschlichen Ebene Respekt also erarbeiten als Professor //l:mhm// aber glaube wenn jemand fachlich also was drauf hat und das zu vermitteln weiß dann ist das äh also didaktisch irgendwie gut ist dann wird das eigentlich immer honoriert bei den Studenten" (S07 49).

„ja und das da fühlt man sich dann einfach besser aufgehoben wenn man merkt die Leute wollen einem wirklich was beibringen" (S03 73).

„wenn er ne Frage gestellt hat und ich die beantworten konnte dann kriegt man ja schon so ein bisschen Anerkennung //I: ja// schon so ein bisschen Lob und ähm weil ich da halt in der Schule auch schon so drauf aus war //I:mhm// so hat mir das halt immer ganz gut getan und mir immer ganz gut gefallen da dann auch ne gute Leistung zu bringe" (S03 61).

„P: ja also wie zum Beispiel mit Herzgeräuschen wenn man gesagt hat oh nee das hör ich jetzt nicht ja jetzt nochmal und dann musst du doch das hören //l:mhm// und dann gleichzeitig 
nochmal das Stethoskop und es war vielleicht auch meine erste Famulatur //l:ja// weil sich das mir besser gemerkt hat aber //l:mhm// dass er sich da einfach mal ne halbe Stunde Zeit genommen hat nur dass ich so ne Aortenstenose hören ähm.. ja fand ich schon obwohl der echt nicht viel Zeit hatte fand ich zum Beispiel total gut //l:ja//und seit wir dann ja dann eben immer wenn was Spannendes war auch Famulanten von denen er jetzt nicht irgendwie Profit hatte oder so //l:mhm// ich glaube es hat inm einfach selber Spaß gemacht und wusste vielleicht wie es bei inm damals war." (S05 075).

„I: Und du meintest du hättest vorher so $\mathrm{n}$ Bild von Professoren gehabt äh wie sie sein würden und er war dann aber ganz anders. P: Ja also ich hab die also persönlich immer gedacht also man also dass die so unantastbar sind //l:hm// und irgendwo dass man sie eigentlich auch ganz selten zu Gesicht bekommt //l:hm// was ja auch irgendwo stimmt //l:mhm// ähm ähm aber ich hab das halt irgendwann erst so verstanden dass es ne dass es immer so ne Ansammlung von Titeln ist und äh dass es aber irgendwie doch bei vielen ja das könnten halt auch mein mein Vater sein oder so ne //l:mhm// also das dementsprechend ne schon ne andere Generation und //I:hm// auch äh noch vorgesetzt irgendwie aber so also lange nicht so so krass wie ich mir das immer so vorgestellt habe" (S07 18).

„und ich finde dass sich das häufig auch son bisschen verbindet dass so Zweck zweckmäßiges Beisammensein aber man sich auch sympathisch findet es aber niemals ausreichen würde dass man jetzt zusammen in Urlaub fährt“ (S12 15)

„das war also eine sehr peinliche Antwort weil ich habe gar nicht verstanden was er gemeint hatte //l:okay// und er hat mich nicht fertig gemacht //l:mhm// er hat einfach andere Frage gestellt und ja das empfahl mir einfach natürlich weiter zu lernen //l:mhm// und ich finde immer noch Herr X-(Name eines Dozenten1) //l: ja// sehr gut“ (S09 11)

„was mich so noch geprägt hat (10) naja vielleicht in Famulaturen ich hab kein konkretes Beispiel aber das zum Beispiel ähm es ein Riesenunterschied für den Patienten macht wie man.. ob man unsicher als Arzt auftritt oder ob man den Patienten Sicherheit vermitteln kann und das find ich war in vielen.. vielen Famulaturen zumindest ziemlich erkennbar //l:mhm// was bestimmt auch mit Erfahrungen äh Berufserfahrung zu tun hat //l:ja// ... und was man in den Famulaturen find ich auch noch gesehen hat dass man ähm.. den Patienten also das ist so ne Gradwanderung ist wie nah man nem Patienten kommen kann //l:mhm//.. das vielleicht noch //I:mhm//... geht es nur um zwischenmenschliche Beziehungen was man im Studium gelernt hat" (S05 003)

„naja dann kann ich ja mal jetzt die gerade die Situation erzählen zumindest die fand ich auch ziemlich ähm... ja.. vorbildmäßig also //l:mhm// eben diese Ärztin die ähm sehr.. menschlich mit den Patienten umging //l:mhm// sie gleichzeitig aber nicht bemitleidet hat oder sie irgendwie als krank hingestellt hat sondern //l:mhm// sie sehr respektvoll behandelt hat" (S05 003).

„P: die waren behutsam //l:mhm// aber man hat schon gemerkt dass ähm das sie damit umgehen können //l:mhm// also dass sie nicht also die waren zum Beispiel zu mir waren sie sehr lieb und nett und haben auch gesagt ich könnte auch nach Hause gehen und ähm ich müsste 
jetzt nicht bleiben //l:mhm// das müsste man erstmal verarbeiten und so und ähm .. genau und ähm und dann aber die waren dann halt die haben sich sehr zurückgehalten weil eben dieser Mann auch noch sehr ähm der musste ja erstmal sich ausweinen //l:mhm// und erstmal so wieder in die Realität zurückkommen und ähm .. ja ich wir als Ärzte haben uns da eher rausgehalten //l:mhm// haben dann dem Mann nochmal erklärt als er dann gefragt hat was dann war" (S11 075)

„wenn wir es nicht wussten //l:mhm// ... was hat er denn dann gesagt manchmal hat er gesagt das müssen sie vorher wissen oder irgend sowas und dann hat er sich ein bisschen naja nee irgendwie haben sie wohl nicht aufgepasst //l:((lacht))// haben sie wohl lange geschlafen oder irgendwie keine Ahnung" (S14 119)

„weil sie die Art wie sie die Atmosphäre geschaffen hat also die Art wie sie dieses Gespräch geführt hat das fand ich halt gut sie hat da Ruhe reingebracht sie hat sich da hingesetzt und sie hat sehr empathisch gewirkt //l:mhm// und gleichzeitig sehr kompetent also dadurch dass sie eben schon so viele Sachen gemacht hat //l:mhm// und so in verschiedene Richtungen gefragt hat und sie hat halt auch sie hat so ähm glaub ich sanft aber bestimmt untersucht so sah es jedenfalls von außen aus" (S11 095)

„ja und der interessiert sich auch für Chirurgie// ja genau also in reizt das ziemlich dass also wie gesagt einmal diese Ellenbogensache auch //l:mhm// dass er um seine OPs kämpfen will //l:mhm// und dass er als als Neurochirurg zum Beispiel oder als Herzchirurg einfach an denen vitalen Organen dran ist //l:mhm// und es da einfach in dem Moment total drauf ankommt dass jetzt.. in dem Moment sehr konzentriert zu machen //l:mhm// und sehr gut zu machen ich glaube auch weil's weil's sowieso in gewisser Hinsicht so also gerade aus dieser Leute Augen auch ne Königsdisziplin ist //I:mhm// hmm also das eins von diesen zwei Sachen möchte er irgendwie schon ganz gerne machen ah ja genau dann vorher war das noch gar nicht so klar mit der Chirurgie da war's erstmal erstmal Notfallmedizin //l:mhm// aber dieses äh jetzt kommt's drauf an Zack Zack Zack Zack Zack das zu machen alles im Kopf zu haben und ähm vielleicht nicht erstmal eingehender über bestimmt Ursachen nachzudenken wie das und das zusammenpasst was mir jetzt erstmal eher liegen würde also so Innere Medizin und eher mit ner umfassenden Diagnostik.. das also ist nicht so sein Ding //l:mhm okay// und dann also hat er mir zum Beispiel neulich erzählt dass er sich jetzt auf ne andere Art und Weise rasiert ((beide lachen)) nämlich wie sich so ein Chirurg also so wie son Chirurg zum Beispiel auch die Schere anfassen würde ((lachen beide)) //l:okay... ja// (5) ja wir haben jetzt leider im Moment gerade kaum Kurse zusammen ne wir haben keinen einzigen Kurs zusammen //l:mhm// deswegen sehe ich den jetzt gar nicht mehr so viel gesehen habe.. aber im Prinzip ist diese äh diese Haltung geblieben dass //l:mhm// alles richtig richtig gut können und am besten können“ (S04 031)

„ja und .. ((lacht)) irgendwie so hm.. also einfach wirklich so diese von der Form her .. das was vielleicht die deutsche Krebshilfe da als Sponsor //l:mhm// wichtig fand bestimmte fancy Sachen zu haben wie son wie son Meditationsraum oder sowas //l:mhm// .. das war ihm dann 
eher auch ein bisschen naja suspekt oder hat ein bisschen.. naja ein bisschen peinlich eher //I:((lacht))// oder er hat einfach gesagt darauf kommt es hier alles nicht an //I:mhm// also er hat einfach das nicht nicht über die Sache hinaus irgendwie ausgestellt sondern war eher darum bemüht zu sagen ja das äh hängen sie mal nicht zu hoch oder so //l:mhm// sondern das irgendwie um die Sache an sich“ (S04 079)

„dass wir uns da eigentlich ham wir uns immer gut austauschen können über Dinge die wir erlebt haben //I:mhm// hat er auch manchmal ähnlich gesehen und das hab ich auch viel von ihm lernen können dass er .. wie soll ich sagen vielleicht noch viel mehr als ich hat er versteht er Medizin glaub ich sehr zwischenmenschlich //I:mhm// sehr .. auf den Menschen bezogen während ich vielleicht die Sachen eher biologischer sehe //l: ja// konnt ich da viel von ihm lernen glaub ich wie man .. dass man halt irgendwie auf die Menschen sehr achten muss bei den Dingen“ (S08 28)

„P: also der war erstmal auch ohne Kittel da... was jetzt aber gar nicht unbedingt die.. die Hauptsache war... ich glaube hm.. vielleicht die Art und Weise wie der gesprochen hat //I:mhm// ... ((lacht)) glaube er.. jetzt wo ich so drüber nachdenke wirkte er schon ein bisschen.. besorgt irgendwie auch also.. //l:mhm// hm... also als als würde das ihn nicht alles so.. kalt lassen //l:mhm// er hat erzählt zum Beispiel dass die da auch ein veranstaltet haben.. Also weil die weil der Patient nicht nach Hause konnte //l:mhm// sind halt jetzt halt die Familie dahin gekommen und haben sie da nen Kindergeburtstag gefeiert und also das das erstmal möglich zu machen und diese Station wirklich erstmal grundsätzlich so zu gestalten dass sie genau sowas da mit einbeziehen“ (S04 075)

„dass er das total korrekt ist mit den Leuten //l:mhm// und empathisch ist und es dann trotzdem versucht zu erklären und ähm das finde ich sehr beeindruckend //l:mhm// wie er das so macht und am liebsten hätte ich ne Doktorarbeit gemacht wenn mich das mehr interessiert hätte aber ((lacht)) //l:mhm// das fand ich echt ähm find ich find ich gut wie der das macht“ (S13 007)

„aber da in der Hinsicht hab ich halt außer diesem Arzt $^{27}$ noch nicht so richtig son Vorbild quasi //I:mhm// für mich gefunden aber das kommt vielleicht noch“ (S14 103)

„mein Doktorvater hat mich sicherlich geprägt //I:mhm// .. war nicht immer ganz leicht vielleicht .. aber hat natürlich auch mir hab ich viel gelernt natürlich durch musste man schon aber das ist vielleicht auch normal in der Doktorarbeit musste man viel Frust ertragen //l: ja// wirst du vielleicht auch noch merken //I: ((lacht))// immer rund läuft das alles nicht und ich glaub man wächst aber dran“ (S08 10)

${ }^{27}$ Der Ausdruck „diesem Arzt“ scheint sich in dem Zitat wiederum auf den Arzt, den sie in X- (Name eines Landes) kennen gelernt hat, zu beziehen, es bleibt in diesem Zusammenhang jedoch nicht eindeutig. 
„auch wenn man dann nur im Weg steht und nur nervt und auch ähm was heißt nervt aber ist ja schon anstrengend ist für alle Beteiligten und die Leute da auch nicht so richtig Bock haben wenn's da abgeht" (S13 052).

„hat mich also hat mich beeindruckt //l:hm// weil das son Professor war der da echt sehr menschlich und ähm //l: $\mathrm{mhm} / /$ (( überlegt leise)) ja vielleicht auch son bisschen vorbildmäßig da //l:ja// und das aber meine persönliche ähm Einschätzung“ (S07 04)

„und dadurch das weich das menschliche total irgendwie im Vordergrund steht (laute Hintergrundgeräusche) früher wollte ich auch mal so ne Ärztin werden“" (S14 083)

„dann meinte ich irgendwie zu ihr ja mir fehlt da ein Punkt und ich bin durchgefallen weil ich immer so äh also ich war halt super aufgeregt //l:mhm// und dann meinte sie nur äh hat auch so den Arm um die Schulter gelegt und meinte es ist nicht so schlimm das war bei ihr auch so und //l:ja// ähm es ähm ich muss einfach mir immer wieder diese Situation vorstellen und ähm Kopfkino laufen lassen damit ich einfach nicht mehr so aufgeregt bin //l:ja// sie hatte das früher auch und sie ähm war auch früher vor jeder Vorlesung noch aufgeregt //l:mhm// und erst nach Jahren hat sich das gelegt und sie meinte das ist eigentlich relativ normal und ich fand es einfach sympathisch erstens dass sie Tipps gibt //l:ja// dass sie sagt wie sie das geregelt hat für sich //l:mhm// und ähm.. ja und äh das auch nicht verachtet hat das man jetzt da //l:mhm// durchfällt obwohl es ja wirklich also ich fand's damals extrem schlimm //I:mhm// und sie das absolut mich nicht so hingestellt hat wie oh sie wissen das nicht //l:ja// sondern ähm das passiert jedem mal //l:mhm// und das hat ja seinen Grund ähm gleichzeitig noch son Tipp mitgegeben hat //I:mhm// und gar nicht irgendwie auf das Wissen getrimmt sondern eher eben auf den Mechanismus wie man damit umgeht //l:ja// und dass das hat mir Mut gemacht und das hat mir glaub ich auch ziemlich viel gebracht" (S05 095)

„ja schon manchmal dann vorgekommen im Präpsaal vor allem im Sommer //l:mhm// also wenn es richtig schlimm drin stinkt dass den Leuten dann nicht gut ging und die ich fand dass sie da dann immer ganz gut umgegangen ist und einmal wurde ihr halt auch schlecht und schwindelig //l:mhm// und das war man hat sich eben auf einer Ebene mehr gefühlt //l:mhm// und es gab auch Hiwis die haben sich so sehr drüber gestellt und so war sie nicht //l:ja// und das war irgendwie ne total nette Atmosphäre //l:mhm// ... also ich finde Hierarchien gibt's ja genug //l:ja// die muss man ja nicht noch künstlich aufbauen in dem man sich aufspielt nur weil man zwei Semester weiter ist //l:ja// und das finde ich haben wir irgendwie total nett hingekriegt da" (S15 027)

„//I: und du fandest inn auch inspirierend// ja .. also wie gesagt gerade diese.. echte Anteilnahme dabei" (S04 079).

„und deswegen dachte ich ich bin son schlechtes Beispiel ((lacht)) //l:((lacht))// mich da auch immer sehr rausgezogen hab //l:mhm// irgendwie also ich identifizier mich überhaupt nicht mit diesem Studium //l:mhm// und deswegen bin ich da auch überhaupt nicht präsent //l: ja// glaub ich //I:mhm// und dass ich mich deswegen auch gar nicht so darauf eingelassen hab //I:mhm// und ich suche weder den also ich bin da irgendwie nicht so //l:mhm// nicht so wirklich dabei ich 
glaub ich zieh mich da selber aktiv viel raus //l:mhm// .. das könnte man sicher auch anders handhaben“ (S12 04).

„dann waren das Begegnungen mit Dozenten die einen vielleicht in dem Moment richtig //I:mhm// gezeigt haben du bist noch gar nicht du kannst noch nichts und du kriegst noch keinen Respekt und //l: ja// man vielleicht daran aber auch gewachsen ist dass man lernen muss auf die Zähne zu beißen und //I:mhm// und zu kämpfen vielleicht das hat man vielleicht aus der Vorklinik und den Dozenten mitgenommen“ (S05 11)

„I: und äh kannst du vielleicht noch von den weiteren Verlauf so ähm von eurer Beziehung erzählen also //P:ja// von der Betreuung $\mathrm{P}: \mathrm{mhm}$ das ist eigentlich ist es dann nämlich nicht so toll wie es sich jetzt gerade anhört //l:okay// ähm und zwar ähm.. dachte ich sie wäre auch sehr zuverlässig wenn ich dann ähm bei ihr ne Doktorarbeit anfange //l:mhm// und das hat sich dann eben nicht so entwickelt also dann //l:okay// stellte sie sich von ner anderen Seite raus wie man sie auf den ersten Blick eben nicht eingeschätzt hat //l:okay// und eher relativ unzuverlässig und ähm.. sie gibt mir schon das Gefühl dass sie total hinter mir steht //l:mhm// und auch immer es ist toll wie ich es mache aber es ist keine Verlass auf sie //l:okay// und das find ich ziemlich //l:ja// schwierig einzuschätzen und ich ich hab's vielleicht darauf geschoben dass sie ursprünglich aus X-(Name eines Landes) kommt dass es da vielleicht eine andere Mentalität ist und //I:mhm// man hier viel zu sensibel ist und viel zu hohe Ansprüche an ne Doktormutter stellt ich weiß es nicht //l:okay// ob es daran liegt.. auf jeden Fall bin ich ein bisschen so ins fallen gelassen worden also ich hab nicht die //l:mhm// auf gar keinen Fall die Unterstützung bekommen wie ich es mir erhofft oder erwartet habe //l:ja okay// und so kann man sich auf den zweiten Blick dann //I:mhm// die Leute nochmal angucken“ (S05 113-115)

„und dieser X-(Name des Arztes) ähm ist jemand der einen immer herzlich einlädt einen der wenigen muss man sagen im Uniklinikum die auch sagen ja wir haben Bock dass ihr mal kommt uns helft obwohl man ja de facto noch nicht so viel kann“ (S13 003)

„und ähm die dann insgesamt auch ähm anfangs in ner Viererkonstellation vier Mädels irgendwie waren und uns dann sozusagen zu viert dann eigentlich durch die Vorklinik geschlagen haben und.. ich dann vor allen mit einer Freundin also X-(Name der Freundin) heißt sie ähm... ja.. also ja.. sie für mich einfach ne ganz wichtige Person geworden ist und ähm ich denke wenn man.. dass wenn ich das irgendwie alleine gemacht hätte so.. dann wär's einfach... ähm viel anstrengender gewesen //l: $\mathrm{mhm} / /$ und es hätte... einen einfach viel mehr mitgenommen auch mehr Unsicherheit geschaffen“ (S12 03)

„ähm ich fand das sehr angenehm dass dass er halt auch nicht die ganze Zeit am Tisch //I:mhm// sondern wir auch hauptsächlich mit den mit den mit den mit den Hiwis warn //I:mhm// und da war's ja auch in Ordnung //l:mhm// da konnte man sowieso alles fragen also die warn locker und es war auf einer Ebene mehr und er war dann schon irgendwie der .. der Prof irgendwie //I:mhm// aber ähm er war dann auch nicht so häufig da so dass man irgendwie das Gefühl hatte man konnte da so Fragen stellen“ (S12 19) 
„I: Sind dir denn irgendwann im Studium mal Leute begegnet die du son bisschen als Vorbilder bezeichnen würdest? //P: Höchstens auf der Sommerakademie mhm //I: auch da// ja aber Vorbild ist glaub ich zu viel gesagt //l: ja// (nicht verständlich) einfach okay so $n$ Weg gibt es und so $\mathrm{n}$ Weg kann ich auch finden //l:mhm// und dann niemals dass ich sage oh genau das möcht ich auch erreichen" (S12 47)

„und dann hat er mal bei einer Kommilitonin die selber gerade Herpes hatte so drauf hingewiesen also hat es so als Beispiel genommen //l:mhm// für also eben für eine Erkrankung und also ohne sie da zu fragen sondern hat sie einfach zum Gegenstand der Diskussion gemacht //l:mhm//.. und als sie dann selber darüber gar nicht genau genaue Auskunft geben konnte.. hat er sie dann noch dafür kritisiert dass sie darüber nicht genau Bescheid weiß //I:mhm// also als Medizinstudentin müsste sie das jetzt wissen weil sie müsste sich ja mit den Dingen auseinander setzen und //l:mhm// um dann Bescheid zu wissen.. also das fand ich einfach sehr erschreckend //I:mhm//.. und dass ich einfach gehört habe wie er das häufiger Leute dann wenn nicht in den normalen Stunden dann zumindest mal im Testat in Tränen ausgebrochen sind“ (S04 051)

„ähm sonst war ich glaub ich ja im ersten Semester noch sehr .. eingeschüchtert eigentlich von den Dozenten //l:mhm// also ich hab da ne sehr große .. Distanz so und also ja ich hatte da auch aber ich hatte auch sehr große Angst irgendwie das alles nicht zu können //l:mhm// oder zu versagen unter unterm wahnsinnigen Druck irgendwie gefühlt“" (S12 04)

„Ähm.. da war ein Mann der war ziemlich dement //l:mhm// und die Krankenschwestern ähm eigentlich alle waren immer total genervt der lag auch im ähm Iso-Zimmer //I:mhm// und irgendwie wollte immer keiner reingehen und der ist ständig ausgerastet und hat immer die Leute gehauen und so //I:mhm// und ähm.. ich fand den aber total faszinierend //l:mhm// weil er ähm auf der einen Seite ein ganz höflicher Herr war und immer von irgendwelchen englischen Tees geredet hat und man gemerkt hat //l:mhm// dass er irgendwie vielleicht auch so ein bisschen so einen feinen Hintergrund hat //l: Okay// und dann aber manchmal total umgeschwungen ist und total obszöne Sachen gesagt hat //l: Okay// und total ausgerastet ist //I:mhm// und mit dem habe ich mich total viel beschäftigt.. und hab dann ähm versucht einen Weg zu finden wie man mit inm umgehen kann dass er nicht also ich hab mich dann total viel mit Demenz beschäftigt //l:mhm// auch warum die Menschen vielleicht nie reagieren //l:mhm// ähm hab dann versucht einen Weg mit dem zu finden ohne ähm zum Beispiel da waren ganz laute Geräusche draußen da hat er gedacht draußen wäre Krieg //l:mhm// und wenn man dann sagt irgendwie da draußen ist kein Krieg dann wird er nur sauer und dann wird er aggressiv und wenn man sagt ja wir sind hier drinnen und hab keine Angst drinnen sind wir sicher und so //I:mhm// und so Sachen hab ich dann ein bisschen mit dem ausprobiert und das war also was heißt ausprobiert das hört sich jetzt irgendwie fies an aber //l:mhm// ich habe mich halt wirklich mit inm beschäftigt und das war sehr besonders //l:ja// für mich.. weil ich da das erste Mal so ein bisschen das Gefühl hatte das macht mir wirklich Spaß und mit den Menschen zu arbeiten mit den Patienten zu arbeiten Weil ich ähm.. das Studium schon immer interessant fand aber irgendwie... ich hätte auch was anderes gemacht //l:mhm// aber das war 
was was ich nicht unbedingt machen wollte und da manchmal musste ich mir den Sinn auch suchen... und das war so eine Begegnung //l:mhm// die dann da so ein bisschen für mich“ (S15 007)

„P: ähm ... ja ich weiß noch dass wir da alle am Anfang im ersten Mal um den Tisch standen und die meisten versucht haben so weit wie möglich weg zu stehen weil sie //I:mhm// es nicht gut aushalten konnten und er hat halt son bisschen erzählt wie wie es einem so gehen kann //l:mhm// wenn man das erste Mal mit der Leiche jetzt die Angst davor die Grenzüberschreitung //I:mhm// und es vor allem ganz schwierig dann ist wenn man am Gesicht eben anfängt das zu präparieren und so das sind ja auch Angst die man vielleicht oder die man selber schon hat vielleicht noch nicht so ausformuliert //I:mhm// im Kopf aber die man schon hat und erkennt diese Ängste wahrscheinlich und hat sich damit viel beschäftigt //l:mhm// und ähm das war einfach gut dann zu hören oder zu merken man ist nicht alleine //l:ja// mit seinen Ängsten sondern alle haben die oder es gibt diese Ängste einfach“ (S15 035)

„also dass man da irgendwie so dieselben Probleme hat... und dass das schon etwas..also etwas befremdlich einfach auch ist //I: $\mathrm{mhm} / /$ einmal..einmal einfach dieses dieses instrumentelle an jemandem herumschneiden und andererseits aber auch die Gewöhnung daran //l:ja// also...und dann hab ich wiederrum auch mit Leuten gesprochen darüber dass es dass es zumindest gut ist dass man sich noch darüber wundert dass man sich so gewöhnt" (S04 055)

„und ähm naja das hat sich halt später in den Tierversuchen auch nochmal ((lacht)) rausgestellt da sprechen irgendwie immer alle möglichen Leute völlig selbstverständlich“ (S04 003)

„die haben irgendwie tatsächlich dann halt gefragt ob einer von uns ein bisschen mit helfen will dann hab ich gesagt na klar mach ich ich helfe gerne und dann durfte ich das Herz mal halten in der Hand //l:mhm// das fand ich zum Beispiel auch super weil der Chirurg meinte dass hatte quasi kein ist fragwürdig ne aber hatte keinen wirklichen medizinischen Grund dieses Herz zu halten //I:mhm// er meinte aber dass ich dass ich das unbedingt mal erlebt haben muss //l:aha// das Herz zu halten ich fand das cool weil (nicht verständlich) seit 15 Jahren //l:mhm// seine Herz-OPs und hat schon ganz oft ein schlagendes Herz (nicht verständlich) und hat es trotzdem noch so als was besonderes empfunden das fand ich cool wo ich manchmal denke vielleicht verliert man oder manche verlieren dann die Faszination (nicht verständlich) zum Beispiel am OP-Tisch im OP also ein Herz das ist dann schon ein bisschen entfremdet vom dem menschlichen zum Teil ne //I:mhm// (und da eben sägen und hämmern und so) //l:mhm// dass das halt trotzdem noch total da ist das fand ich ziemlich cool zu sehen." (S14 111)

„ja mir ist grade noch einer eingefallen ne Dozentin doch die die ich wirklich ganz cool fand .. ähm //l:mhm// die hat den Untersuchungskurs Innere bei uns gemacht //l:mhm// war eben ne Onkologin hat auf Onkologie gearbeitet und war auch schon wieder das finde ich auch fällt mir grad so auf dass mir diese auch so die hat irgendwie unglaublich also das ist ja unglaubliches Leid da auf dieser Station //l:mhm// die war auch irgendwie so sehr tough" (S14 159) 
„Also ich glaube dass ich generell irgendwie sehr wenig Kontakt .. habe und hatte irgendwie ähm .. zu Dozenten //I:mhm// oder zu Professoren also ich hab den Kontakt zu Studierenden oder zu mir irgendwie nie also überhaupt nie irgendwie als nah //l:mhm// erlebt mit einer Ausnahme und ich glaub der also der erste Kontakt an den ich mich erinnern kann das war tatsächlich ne Kommilitonin weil mir mir ging's halt so dass //l:mhm// dass ich irgendwie ganz wenig Wissen hatte noch von dem Studium also ich ich hatte irgendwie kein kaum Biologieunterricht und so //I:mhm// und hatte total die Schwierigkeiten mit Chemie und Bio und so weiter .. und da hab ich im ersten Semester irgendeine angesprochen //I:mhm// und die hat mir da ... da wirklich weiter geholfen" (S12 04).

„P: nja also dieser X-(Name Professors) ist irgendwie so.. jemand vom dem ich ne ganze Menge Schlechtes gehört habe dass der Leute an seinem Präptisch ziemlich fertig gemacht hat dass er auch zum Teil mal schwierige Fragen gestellt hat und eigentlich eher wollte dass jemand die nicht beantwortet //l:mhm// also irgendjemand hat der zum Beispiel.. eigentlich nicht so richtig drangekriegt weil er dann eine wohl hm eigentlich schwierig zu beantwortende Frage gestellt hat und diese Person inm aber immer Paroli bieten konnte //l:mhm// und er dann den Rest der Stunde wohl eher also beleidigt gewesen sein“ (S04 051) 


\subsection{Transkriptionsregeln}

\begin{tabular}{|c|c|}
\hline Transkriptionszeichen & Erklärung \\
\hline & $\begin{array}{l}\text { Gesprächspausen wurden durch Punkte } \\
\text { gekennzeichnet. Ein Punkt steht für eine } \\
\text { kurze Pause (unter einer Sekunde). }\end{array}$ \\
\hline$\ldots(4)$ & $\begin{array}{l}\text { Längere Pause wurden durch mehrere } \\
\text { Punkte gekennzeichnet, gefolgt von der } \\
\text { Zahl, die die Dauer der Pause in Sekun- } \\
\text { den beinhaltet (z.B. (4) für eine Pause } \\
\text { von } 4 \text { Sekunden). }\end{array}$ \\
\hline Wort- & $\begin{array}{l}\text { Wortabbruch: Macht den Anfang eines } \\
\text { Wortes kenntlich, das nicht zu Ende ge- } \\
\text { sprochen wurde. }\end{array}$ \\
\hline (sagte er) & $\begin{array}{l}\text { Unsichere Transkription: Bedeutet, dass } \\
\text { das Gesagte schwer verständlich war } \\
\text { und die Transkription mutmaßlich erfolg- } \\
\text { te. }\end{array}$ \\
\hline ( ) & $\begin{array}{l}\text { Leere Klammern stehen dafür, dass Ge- } \\
\text { sprochenes unverständlich ist und somit } \\
\text { ein Teil fehlt. }\end{array}$ \\
\hline$(($ lacht $))$ & $\begin{array}{l}\text { Akustische Ereignisse wie Geräusche, } \\
\text { die die Interviewten machten, wurden } \\
\text { durch doppelte Klammern kenntlich ge- } \\
\text { macht (wenn diese z.B. lachten, wurde } \\
\text { dies als ((lacht)) im Text dokumentiert). }\end{array}$ \\
\hline (Freundin1) & $\begin{array}{l}\text { Zur Anonymisierung wurden die Namen } \\
\text { der genannten Personen nicht transkri- }\end{array}$ \\
\hline
\end{tabular}


Anhang

\begin{tabular}{|l|l|}
\hline & $\begin{array}{l}\text { biert. Anstelle dessen wurde ihre Funkti- } \\
\text { on eingefügt. Wurden mehrere Personen } \\
\text { mit gleicher Funktion für die Interviewten } \\
\text { erwähnt, wurden diese nummeriert, um } \\
\text { kenntlich zu machen, dass es sich um } \\
\text { unterschiedliche Personen handelt (z.B. } \\
\text { Freundin1, Freundin2...) }\end{array}$ \\
\hline kursiv und fett geschrieben & $\begin{array}{l}\text { Kursiv und fett geschriebener Text kenn- } \\
\text { zeichnet von der Interviewerin gespro- } \\
\text { chene Textteile (bzw. auch Geräusche } \\
\text { s.o.). }\end{array}$ \\
\hline // // & $\begin{array}{l}\text { Doppelte Spiegelstriche weisen auf ei- } \\
\text { nen Sprecherwechsel hin. }\end{array}$ \\
\hline
\end{tabular}




\subsection{Reduktionstabelle der Hauptkategorien}

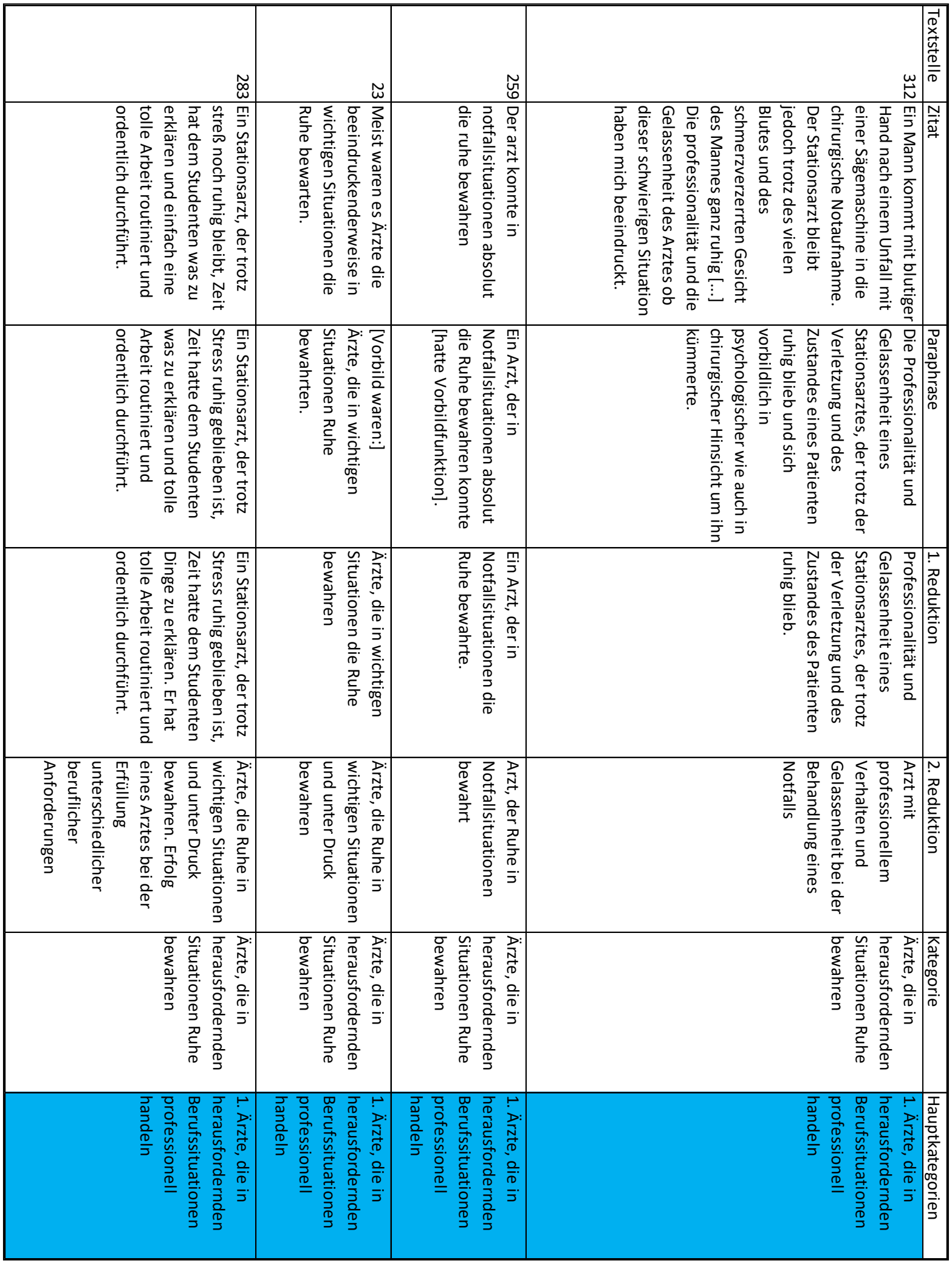

Abbildung 10: Die Tabelle zeigt den Ablauf der Kategorienbildung durch Reduktion. Die gewählten Zitate sind Beispiele für Textstellen, die alle der gleichen Kategorie zugeordnet wurden. Diese Kategorie führte zusammen mit anderen zur Bildung einer Hauptkategorie (vgl. Anhang 8.2 Liste der Hauptkategorien). 


\subsection{Beispiel einer Mind-Map zum Phänomen Ideal}

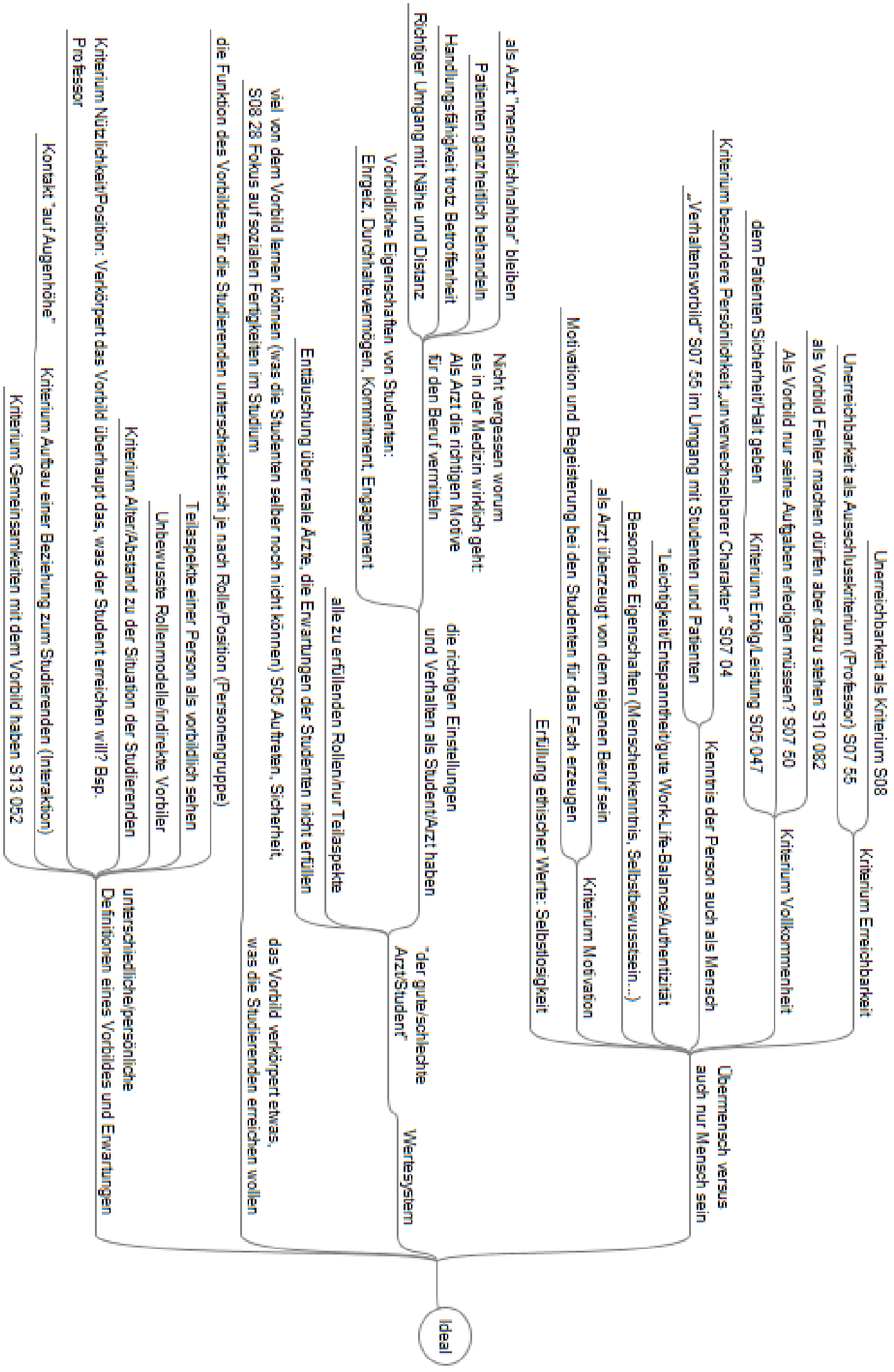

Abbildung 11: Die Abbildung zeigt wie zur Arbeit am Datenmaterial Phänomene in Form von Mind-Maps dargestellt und deren Aspekte konzeptuell in Beziehung gesetzt wurden. 


\section{$9 \quad$ Literaturverzeichnis}

Arnold M: Schwerpunkt: Personal (Krankenhaus-Report 2001). Schattauer, Stuttgart 2002

Asghari F, Fard NN, Atabaki A (2011): Are we proper role models for students? Interns' perception of faculty and residents' professional behaviour. Postgrad Med $\mathrm{J}$ $\underline{87}, 519-523$

Baldwin DC, Daugherty SR, Eckenfels EJ (1988): Student perceptions of mistreatment and harassment during medical school. A survey of ten United States schools. West J Med 155(2), 140-145

Bandura A, Ross D, Ross SA (1963): Imitation of film-mediated aggressive models. J Abnorm Psychol $\underline{66}$, 3-11

Becker HS: Boys in White: Student culture in medical school. Transaction Books, New Brunswick 1977

Benbassat $J$ (2014): Role modeling in medical education: the importance of a reflective imitation. Acad Med $4,550-554$

Bjorksten O, Sutherland S, Miller C, Stewart T (1983): Identification of medical student problems and comparison with those of other students. J Med Educ $\underline{58}, 759$ 767

Böhle F, Voß G, Wachter G (Hrsg.): Handbuch Arbeitssoziologie. Verlag für Sozialwissenschaften, Wiesbaden 2008

Bohnsack R, Marotzki W, Meuser M: Hauptbegriffe qualitative Sozialforschung: Ein Wörterbuch. Leske + Budrich, Opladen 2003

Brüsemeister T: Qualitative Forschung: Ein Überblick. 1. Auflage; Westdt. Verlag, Wiesbaden 2000

Bulte C, Betts A, Garner K, Durning S (2007): Student teaching: views of student near-peer teachers and learners: Medical Teacher. Med Teach $\underline{6}, 583-590$

Burkart G (1983): Zur Mikroanalyse universitärer Sozialisation im Medizinstudium: Eine Anwendung der Methode der objektiv-hermeneutischen Textinterpretation. Z Soziol, 24-48

Cruess RL, Cruess SR, Boudreau JD, Snell L, Steinert Y (2015): A schematic representation of the professional identity formation and socialization of medical students and residents: a guide for medical educators. Acad Med $\underline{6}, 718-725$

Cruess SR, Cruess RL (2012): Teaching professionalism - Why, What and How. Facts Views Vis Obgyn 4, 259-265

Drouin J, Denis M, Nadeau L, Chénier Y (2006): Medical students as teachers and role models for their future colleagues. Med Teach $\underline{7}, 618-624$

Esser H (1986): Über die Teilnahme an Befragungen. ZUMA Nachrichten 18, 38-47 [urn:nbn:de:0168-ssoar-210300 06.03.2016]

Flexner A: Medical education in Europe: a report to the Carnegie Foundation for the Advancement of Teaching, New York City 1912 
Literaturverzeichnis

Flick U, von Kardorff E, Steinke I: Qualitative Forschung: Ein Handbuch. 5. Auflage; Rowohlt-Taschenbuch-Verlag, Reinbek bei Hamburg 2007

Friedrichs J, Mayer KU, Schluchter W: Soziologische Theorie und Empirie. VS Verlag für Sozialwissenschaften, Wiesbaden 1997

Gágyor I, Hilbert N, Chenot JF: Wie häufig und belastend sind negative Erfahrungen im Medizinstudium? Ergebnisse einer Online-Befragung von Medizinstudierenden. GMS Z Med Ausbild 2012; 29: Doc55

Gaufberg EH, Batalden M, Sands R, Bell SK (2010): The Hidden Curriculum: What Can We Learn From Third-Year Medical Student Narrative Reflections? Acad Med $\underline{11}, 1709-1716$

Goffman E: Interaktion: Spass am Spiel, Rollendistanz. R. Piper, München 1973 Hafferty FW, Franks R (1994): The hidden curriculum, ethics teaching, and the structure of medical education. Acad Med 11, 861-871

Haidet P, Stein HF (2006): The Role of the Student-Teacher Relationship in the Formation of Physicians. The Hidden Curriculum as Process. J Gen Intern Med S1, S16

Haidet P, Hatem DS, Fecile ML, Stein HF, Haley HA, Kimmel B, Mossbarger DL, Inui TS (2008): The role of relationships in the professional formation of physicians: Case report and illustration of an elicitation technique. Patient Educ Couns $\underline{3}, 382-387$

Herrmann M (2011): Relevanz der Salutogenese in der medizinischen Ausbildung Ein möglicher Beitrag für die ärztliche Professionsentwicklung. Zukunft der Hochschul-Bildung der Gesundheitsberufe III: Wie können aus Gesundheitsberufen Gesundheitsberufe werden? 28-34, o. Verl.

Hilbert N: Negativer Rückblick oder positive Aussicht? - eine Online-Befragung Studierender der Medizin über ihre Erfahrungen während des Studiums. Med. Diss. Göttingen 2012

Hudson JN, Tonkin AL (2008): Clinical skills education: outcomes of relationships between junior medical students, senior peers and simulated patients. Med Educ $\underline{9}$, 901-908

Hundert EM, Hafferty F, Christakis D (1996): Characteristics of the informal curriculum and trainees' ethical choices. Acad Med $\underline{6}$, 624-642

Ibrahim T (2004): The case for invigorating internal medicine. Am J Med $\underline{5}$, 365-369

Jarvis-Selinger S, Pratt DD, Regehr G (2012): Competency is not enough: integrating identity formation into the medical education discourse. Acad Med $\underline{9}, 1185-1190$

Karnieli-Miller O, Vu TR, Holtman MC, Clyman SG, Inui TS (2010): Medical students' professionalism narratives: a window on the informal and hidden curriculum. Acad Med 1, 124-133

Kenny NP, Mann KV, MacLeod H (2003): Role modeling in physicians' professional formation: reconsidering an essential but untapped educational strategy. Acad Med $\underline{12}, 1203-1210$

Lamnek S: Qualitative Sozialforschung. Psychologie-Verlags-Union, München 19881989

Lamnek S: Qualitative Sozialforschung: Lehrbuch. 4. Auflage; Beltz, Weinheim 2005 
Lockspeiser TM, O'Sullivan P, Teherani A, Muller J (2008): Understanding the experience of being taught by peers: the value of social and cognitive congruence. Adv Health Sci Educ Theory Pract $\underline{3}, 361-372$

Mark R (2014): Sie fragen nach der Lehre? Wie schön!: Über einige Unterschiede zwischen deutschen und amerikanischen Universitäten. FAZ $\underline{233}$ 08.10.2014

Marx G WA (2009a): Qualitative Sozialforschung - Ausgangspunkte und Ansätze für eine forschende Allgemeinmedizin: Teil 1: Theorie und Grundlagen der qualitativen Forschung. DEGAM-NACHRICHTEN, 105-113

Marx G WA (2009b): Qualitative Sozialforschung - Ausgangspunkte und Ansätze für eine forschende Allgemeinmedizin: Teil 2: Qualitative Inhaltsanalyse vs. Grounded Theory. DEGAM-NACHRICHTEN, 467-475

Maudsley RF (2001): Role models and the learning environment: essential elements in effective medical education. Acad Med $\underline{5}$, 432-434

Mayring P: Einführung in die qualitative Sozialforschung: Eine Anleitung zu qualitativem Denken. 5. Auflage; Beltz, Weinheim 2002

Mayring P: Qualitative Inhaltsanalyse: Grundlagen und Techniken. 11. Auflage; Beltz, Weinheim 2010

Merton RK: The student-physician: introductory studies in the sociology of medical education; Harvard University Press, Cambridge 1957

Meyer T, Karbach U, Holmberg C, Güthlin C, Patzelt C, Stamer M (2012): Qualitative Studien in der Versorgungsforschung-Diskussionspapier, Teil 1:

Gegenstandsbestimmung. Gesundheitswesen, 74(08/09): 510-515

Moebius S, Albrecht C: Kultur-Soziologie: Klassische Texte der neueren deutschen Kultursoziologie. Imprint: Springer VS, Wiesbaden 2014

Murray HA: Explorations in personality. 70. Auflage; Oxford University Press, Oxford 2008

Omer H, Schlippe Av: Stärke statt Macht: Neue Autorität in Familie, Schule und Gemeinde. 1. Auflage; Vandenhoeck \& Ruprecht, Göttingen 2010

Parsons T (1958): Struktur und Funktion der modernen Medizin. Eine soziologische Analyse. Kölner Z Soz Sozpsychol, Sonderheft, 3

Polanyi M: Implizites Wissen (Suhrkamp Taschenbuch Wissenschaft 543).

Suhrkamp, Frankfurt am Main 1985

Richards H, Emslie C (2000): The 'doctor' or the 'girl from the university'?

Considering the influence of professional roles on qualitative interviewing. Fam Pract $\underline{17}, 71-75$

Rosenthal G: Interpretative Sozialforschung: Eine Einführung. Juventa, Weinheim 2005

Schrauth M, Kowalski A, Weyrich P (2009): Selbstbild, Arztbild und Arztideal: Ein Vergleich Medizinstudierender 1981 und 2006. Psychother Psychosom Med Psychol 12, 446-453

Shuval JT, Adler I (1980): The role of models in professional socialization. Soc Sci Med 1, 5-14 
Strauss A, Corbin J: Grounded Theory: Grundlagen qualitativer Sozialforschung. Beltz, Weinheim 1996

Szauter K, Williams B, Ainsworth MA, Callaway M, Bulik R, Camp MG (2009):

Student perceptions of the professional behavior of faculty physicians. Med Educ Online $\underline{8}, 1-5$

Ten Cate O, Durning S (2007): Peer teaching in medical education: twelve reasons to move from theory to practice. Med Teach $\underline{6}, 591-599$

Terzioglu P, Jonitz B, Schwantes U, Burger W (2003): Vermittlung muss im Medizinstudium beginnen: Die Fähigkeit des Arztes zu einer gelungenen Kommunikation mit dem Patienten ist eine wichtige Voraussetzung für eine erfolgreiche Therapie. Dtsch Arztebl 10, 458-460

Weissmann PF, Branch WT, Gracey CF, Haidet P, Frankel RM (2006): Role Modeling Humanistic Behavior: Learning Bedside Manner from the Experts. Acad Med $\underline{7}, 661-667$

Wiegelmann G: Geschichte der Alltagskultur: Aufgaben und neue Ansätze (Beiträge zur Volkskultur in Nordwestdeutschland 21). F. Coppenrath, Münster 1980

Wilmanns JC (2003): Die ersten Krankenhäuser der Welt: Sanitätsdienst des Römischen Reiches schuf erstmals professionelle medizinische Versorgung. Dtsch Arztebl $\underline{40}, 2592-2597$

Witte N: Ärztliches Handeln im Praxisalltag: Eine interaktions- und biographieanalytische Studie (Reihe "Biographie- und Lebensweltforschung" des Interuniversitären Netzwerkes Biographie- und Lebensweltforschung). CampusVerlag, Frankfurt am Main 2010

Witte N: Typenbildung als theoretische Verallgemeinerung. Zitiert nach Inhaltsangabe des Vortrages (gehalten 13.03.2015). Mildred Scheel Akademie Göttingen

Wright S, Carrese $J$ (2002): Excellence in role modelling: insight and perspectives from the pros. CMAJ $\underline{6}, 638-643$

Wright S, Wong A, Newill C (1997): The impact of role models on medical students. J Gen Intern Med 1, 53-56

Wright S, Kern DE, Kolodner K, Howard DM, Brancati FL (1998): Attributes of excellent attending-physician role models. N Engl J Med 27, 1986-1993 


\section{Danksagung}

Mein besonderer Dank gilt Frau Dr. Ildikó Gágyor für ihre zuverlässige Betreuung und die fortwährende und engagierte Unterstützung und Herrn Prof. Dr. Wolfgang Himmel für die kompetente und bereichernde Betreuung.

Des Weiteren auch ein herzlicher Dank an die wissenschaftlichen Mitarbeiter/innen des Instituts für Allgemeinmedizin für ihre Hilfsbereitschaft und stets freundliche Unterstützung.

Ganz herzlich möchte ich auch Maya Hasenbeck, Eckart und Hajo Bücken für die Korrekturen dieses Manuskriptes und Marlon Krug für seine unermüdliche Verfügbarkeit für technische Fragen danken. 